\title{
Exploring A Bioinformatics Clustering Algorithm
}

By

Mukhlis Matti

\author{
A Thesis Submitted To \\ Victoria University Of Wellington \\ In Fulfilment Of The Requirements For The Degree Of \\ Master Of Science \\ In Computer Science
}

Wellington, New Zealand October 2004 


\section{Abstract}

This thesis explores and evaluates MAXCCLUS, a bioinformatics clustering algorithm, which was designed to be used to cluster genes from microarray experimental data. MAXCCLUS does the clustering of genes depending on the textual data that describe the genes. MAXCCLUS attempts to create clusters of which it selects only the statistically significant clusters by running a significance test. It then attempts to generalise these clusters by using a simple greedy generalisation algorithm. We explore the behaviour of MAXCCLUS by running several clustering experiments that investigate various modifications to MAXCCLUS and its data. The thesis shows (a) that using the simple generalisation algorithm of MAXCCLUS gives better result than using an exhaustive search algorithm for generalisation, (b) the significance test that MAXCCLUS uses needs to be modified to take into consideration the dependency of some genes on other genes functionally, (c) it is advantageous to delete the non domain-relevant textual data that describe the genes but disadvantageous to add more textual data to describe the genes, and (d) that MAXCCLUS behaves poorly when it attempts to cluster genes that have adjacent categories instead of having two distinct categories only. 


\section{Acknowledgments}

Firstly, I would like to thank God for helping me and giving me the opportunity to contribute something to human civilisation by letting me to do and finish the research project and write the thesis.

Secondly, I would like to thank my supervisor: Dr Peter Andreae, for his valuable feedback, effort, time, and support.

I would like also to thank the following people:

From Victoria University of Wellington:

From the School of Mathematics, Statistics \& Computer Science:

Dr John Haywood and Dr Richard Arnold.

The people that take care of the school's computer systems:

Dr Ray Brownrigg, Chang Chui, Andrew Mellanby, Kirk Jackson, Duncan McEwan, Mark Davies, and Roger Cliffe.

The school administrative staff:

Dr Peter Donelan (Head of School), Millie Byrne, Prema Ram, Elsie Gatfield, Rowan McCaffery, and Ginny Nikorima.

From the School of Biological Sciences:

Dr Rod Lea and Dr Darren J Day.

The staff members of the Faculty of Science Student Administration Office.

\section{From outside Victoria University of Wellington:}

Dr Christine M Morris, Associate Professor and Director, Cancer Genetics Research Group, Department of Pathology, Christchurch School of Medicine, Christchurch, New Zealand.

Dr Don Love, Associate Professor, Functional \& Chemical Genomics Laboratory, School of Biological Sciences, The University of Auckland, Auckland, New Zealand.

The staff members of:

Wellington Hospital Laboratory, Wellington, New Zealand.

Ministry of Health, Wellington, New Zealand.

Medical Laboratory - Wellington, Wellington, New Zealand.

At the end, I would like to thank my parents, my sister, her husband, and my niece for their valuable support. 


\section{Table of contents}

Abstract ii

Acknowledgments iii

1 Introduction 1

1.1 Outline of the Thesis 2

1.2 Clustering 4

1.3 MAXCCLUS 5

1.4 Input data types to MAXCCLUS 5

1.5 Pre-processing textual data 9

1.6 How MAXCCLUS works 10

1.7 How MAXCCLUS reports its clustering results 17

2 Methodology 19

2.1 The clustering experiments 19

2.2 Comparing clustering experiments results 22

2.3 How much of the data can MAXCCLUS describe 26

3 Exhaustive Search Generalisation 27

3.1 Data source 28

3.2 Comparison between two versions of MAXCCLUS 28

3.3 Experimental results 34

3.4 Analysis and discussion 42

3.5 Conclusion 43

3.6 Recommendations 43

4 Operons 45

4.1 Example 47

4.2 Preparing the data 48

4.3 Experimental results 51

4.4 Analysis and discussion 66

4.5 Conclusion 71

4.6 Recommendations 72

5 Deleting Descriptors 73

5.1 The experiment 73

5.2 The algorithm for deleting descriptors 75

5.3 Experimental results 77

5.4 Analysis and discussion 90

5.5 Conclusion 95

5.6 Recommendations 95 
6 Adding descriptors 97

6.1 The purpose of the experiment 97

6.2 Adding descriptors 98

6.3 Experimental results 100

6.4 Analysis and discussion 113

6.5 Conclusion and recommendations 118

7 Five Categories 119

7.1 Textual data 120

7.2 Experimental results 120

7.3 Analysis and discussion 132

7.4 Conclusion 137

7.5 Recommendations 137

8 Tools 139

8.1 Modifications to MAXCCLUS and its data 139

8.2 Comparison used in methodology 139

8.3 Rendering 140

8.4 Web robot 140

8.5 Clusters visualisation 141

9 Conclusion 145

Appendix: The words of the Bible in "Simple English" 149

Bibliography 157 


\section{Chapter 1}

\section{Introduction}

This thesis explores MAXCCLUS, a bioinformatics clustering algorithm, by running a set of clustering experiments to study and analyse MAXCCLUS's behaviour, in order to identify the strengths and limitations of MAXCCLUS. The results of the experiments show that in general MAXCCLUS is doing a reasonably good job.

Clustering is the task of grouping instances into sets so that each set reflects the shared properties between its instances, providing that instances of other sets do not have all these properties.

Clustering as an approach of machine learning is applied in many fields. One of the important applications of clustering is in the field of bioinformatics. Bioinformatics is a branch of science that deals with the collection, storage, classification and analyses of the biological and biomedical information. One of the promising technologies used in bioinformatics is the microarray (also called gene chip) [Molla, M; Waddell, M; Page, D. and Shavlik, J. 2004]. Microarray biotechnology measures gene expression levels for thousands of genes in the same time and in a cost effective way. Gene expression level is the amount of protein that the gene produces. Comparing the gene expression rates before and after a specific event applied to biological cells or tissues (some examples are: antibiotic shock, heating, or cooling) during biological or biomedical experiments, helps the scientists to understand the genes functionality and gives them an insight into what is happening during these experiments. 
Microarray gene expression data are produced around the world in huge amounts. This data is by no mean easy to analyse manually. Therefore, there is a need for automated techniques to do the job. Clustering is one of the automated techniques that can be handed to a machine (a computer) to cluster the data to groups depending on properties that this data share.

An important source of information for such automated techniques is the data about genes in large shared text databases such as Swiss-Prot [Swiss-Prot].

\subsection{Outline of the Thesis}

The rest of this chapter introduces the background to the thesis, and describes the MAXCCLUS algorithm in detail.

In chapter two of this thesis, we describe the methodology we used to analyse MAXCCLUS and the results of the clustering experiments. At the end of chapter two we show how much of the data the original MAXCCLUS (without any changes to it) is able to describe.

In chapter three, we explore MAXCCLUS's simple generalisation approach, and we introduce a new generalisation approach, which is much powerful and much more expensive. The result of running the clustering experiment of chapter three is to show that MAXCCLUS's simple generalisation is better than the introduced one, by being able to explain the same genes in much less time.

In chapter four, we explore the significance test that MAXCCLUS depends on to select the clusters that are statistically significant from the total clusters it creates. The result of running the clustering experiment of chapter four is to show that MAXCCLUS is ignoring the fact that some instances are dependent 
on other ones, which leads MAXCCLUS to select clusters that bigger than they should be to be considered statistically significant. Therefore the result suggests that the significance test should be changed to take into its account the dependency of some instances on other ones.

In chapter five, we explore deleting some of the textual data, which describe the instances that MAXCCLUS attempts to cluster. The result of running the clustering experiment of chapter five shows that it is advantageous to delete some of the textual data providing that it is not domain-relevant. Deleting this data lets MAXCCLUS characterise the clusters it creates in a more scientifically informative way, and to describe almost the same number of genes faster.

In chapter six, also we explore the textual data. This time we attempt to add more textual data (synonyms and/or hypernyms of already existing textual data) to describe the instances. The result of running the clustering experiment of chapter six is to show that it is a bad idea to add more textual data: MAXCCLUS is not able to describe any more genes than before adding more textual data, it creates many alternative characterisations for the clusters that can confuse the user who is trying to make sense of the clusters, and it took much longer to cluster.

In chapter seven, we explore the behaviour of MAXCCLUS when it attempts to cluster instances that have continuous range of categories instead of having two categories separated with a clear gap (the ones that MAXCCLUS originally uses). The result of running the clustering experiment of chapter seven is to show that MAXCCLUS, like some other clustering algorithms, is not able to produce as good results when clustering using a continuous range of categories as when clustering using two distinct categories.

In chapter eight, we briefly describe several tools that we developed for the project of this thesis.

At the end, in chapter nine, we give the overall conclusions for this thesis. 


\subsection{Clustering}

There are many approaches to clustering [Fasulo, D. 1999]. Two of the popular approaches are: $\mathrm{K}$-means clustering, and Agglomerative clustering.

\section{K-means clustering}

In k-means clustering [Falkenauer E. and Marchand A. 2001], the algorithm needs in advance the number of clusters $(k)$ that it will cluster the instances to. The algorithm initially randomly assigns $k$ cluster centres. Next the algorithm clusters each instance to the cluster whose centre is nearest to this instance. Then the algorithm changes each cluster centre to the mean of the centres of its instances. The last two steps repeat until no changes occur any more. K-means clustering assumes numerical attributes because it must be able to compute the cluster mean.

\section{Agglomerative clustering (bottom up)}

In agglomerative clustering, the algorithm constructs a tree out of the instances to be clustered. Initially each one of the instances presents a cluster of its own. Then the algorithm finds the closest two clusters and merges them into a new cluster, which represents the parent cluster of the two merged clusters. This step repeats until there are no clusters left to merge. This kind of agglomerative clustering is called single linkage (also referred to as nearest neighbour). Agglomerative clustering assumes a distance measure can be computed. 


\subsection{MAXCCLUS}

MAXCCLUS is a clustering algorithm designed and implemented by Peter Andreae that uses a novel approach for clustering instances [Andreae, P; Shavlik, J; and Molla M. 2001]. This clustering algorithm is designed to cluster the genes of a microarray experiment to find characterisations of subsets of the genes that behave in the same way during the microarray experiment. The goal behind designing MAXCCLUS is to make sense of the data that the microarray experiment produces, so that scientists can interpret the data more easily by having a clearer view of what is happening in the microarray experiments.

\subsection{Input data types to MAXCCLUS}

MAXCCLUS depends on two types of data to cluster instances: experimental data and textual data.

\subsubsection{Experimental data}

The experimental data that is used, as an input to MAXCCLUS, are actual experimental data from microarray experiments. These experiments are from the University of Wisconsin, Madison, USA. They are a set of 46 experiments on E-Coli, a kind of bacteria. Each microarray experiment dataset is about the genes (the instances) that were involved in the microarray experiment. The experimental data is a table (example: Table 1.1) in which each row contains the instance ID, and a number that represents the expression ratio, which describes the regulation of this gene as a result of this microarray experiment, under a physical or chemical event, such as antibiotic shock or temperature shock. This expression ratio in fact represents the category of the regulation that this instance belongs to, under this microarray experiment. If the expression ratio is greater than 2.0, we say that the instance is Up regulated. 
If the expression ratio is less than 0.5 , we say that the instance is Down regulated. If the expression ratio is in the range [0.5,2.0], this means that this expression ratio is uncertain; we will consider it unreliable and we will ignore it, and the gene will not be included in the experimental input data to MAXCCLUS.

So practically, we can replace the expression ratio of Table 1.1, by its category as in Table 1.2. This makes each microarray experiment dataset be a table (Table 1.2) in which each row contains the instance ID, and the instance category (Up or Down).

Notice that instances with IDs b0003 and b0004 are not included in Table 1.2, because their expression ratios are 1.7 and 0.6 respectively (Table 1.1 ), which make these expression ratios uncertain.

\begin{tabular}{|c|c|}
\hline Instance ID & Expression ratio \\
\hline b0001 & 2.7 \\
\hline b0002 & 0.3 \\
\hline b0003 & 1.7 \\
\hline b0004 & 0.6 \\
\hline b0005 & 3.2 \\
\hline b0006 & 0.4 \\
\hline b0007 & 0.15 \\
\hline
\end{tabular}

Table 1.1: An experimental data source example. This experimental data source is a table in which each row contains the instance ID and a number that represents the expression ratio.

\begin{tabular}{|c|c|}
\hline Instance ID & Category \\
\hline b0001 & Up \\
\hline b0002 & Down \\
\hline b0005 & Up \\
\hline b0006 & Down \\
\hline b0007 & Down \\
\hline
\end{tabular}

Table 1.2: The same experimental data source example of Table 1.1, but the expression ratios were replaced by their categories. This table (Table 1.2) does not include the instances that have uncertain expression ratios (b0003 and b0004). The data in this table (Table 1.2) will be the experimental data input to the clustering algorithms. 


\subsubsection{The textual data}

The other type of data that MAXCCLUS uses, when attempting to cluster instances, is the textual data. The textual data presents the text that annotates the genes that is available in some fields of the databases about these genes. Swiss-Prot [Swiss-Prot] is one of these databases. Swiss-Prot is a protein knowledgebase. Proteins are the life building blocks that genes code for. Swiss-Prot lists the genes with text fields. Figure 1.1 shows a snapshot of the Swiss-Prot database showing the fields for the protein corresponding to gene b0003. Each line has a type [Swiss-Prot User Manual]. Not all the text fields are useful for the purpose of creating the textual data that MAXCCLUS needs to use. For example the last few lines of the field CC are the copyright notice and they are repeated for all the genes. Including the same textual data for all the genes is not useful because it will not discriminate between the genes. 


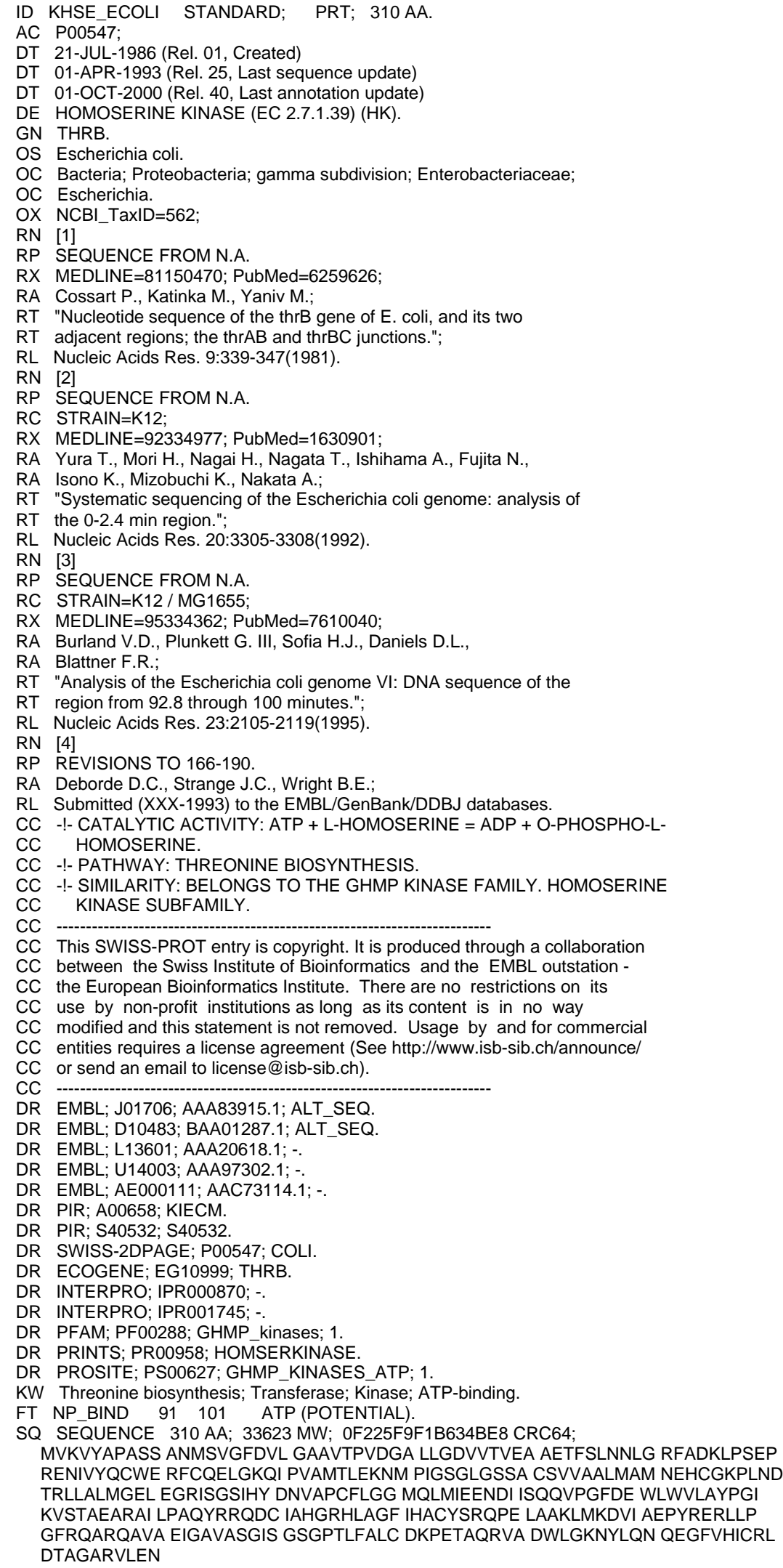

Figure 1.1: A snapshot of the text annotated to genes from Swiss-Prot database. The snapshot is for the gene b0003. 


\subsection{Pre-processing textual data}

This section describes the original pre-processing of the textual data.

The raw data of Swiss-Prot needs to be pre-processed to prepare the textual data for MAXCCLUS. Some of the modifications made to the raw data are:

- Select the $D E, K W, R T$, and $C C$ fields from the Swiss-Prot entries.

- Convert the text to lowercase letters.

- Remove the copyright notice from the CC lines, and some of the reference title lines $(R T)$ that appears in the text of most of the genes.

- Break sentences and phrases into their words. For example "Escherichia coli" becomes two words "escherichia" and "coli".

- Break the "words" that have hierarchical structure into their substructures including the original full structure. For example "EC 2.7.1.39" becomes “ec@2@7@1@39”, “ec@2@7@1", “ec@2@7”, and "ec@2". The use of "@” instead of "." is because all punctuation symbols are to be removed.

- Remove punctuation.

- Replace words with their stems using the Porter stemmer algorithm [Porter, M. F. 1980] that removes suffixes and prefixes from words, because words that have the same stem usually have similar meanings.

- Remove duplicate words in each entry.

After this all the words that belong to a gene comprise the set of descriptors to this gene. The textual data consists of a table in which each row in the table contains the gene id associated with the gene descriptor set (as in Table 1.3). 


\begin{tabular}{|c|l|}
\hline Instance ID & Descriptors \\
\hline b0001 & acid biosynthesis escherichia isoleucine mutant region structural sulfate ... \\
\hline b0002 & active aspartate belong cytoplasmic h(2)o kda molecular pathway protein ... \\
\hline b0003 & adjacent adp analysis atp belong biosynthesis coli ec@2@7@1@39... \\
\hline b0004 & coli compared enzyme mutant observed protein region sulfate transferase .... \\
\hline b0005 & abundance dna-binding encoded homodimer integral orthophosphate ... \\
\hline b0006 & chromatography hypothetical membrane nucleotide predicts subunit sulfate ... \\
\hline b0007 & 51.7 acid analysis biosynthesis coli oxidoreductase potential regulatory ... \\
\hline
\end{tabular}

Table 1.3: A snapshot of the descriptors table showing instances and their descriptors. The text data in this table is the textual data input to MAXCCLUS.

When the experimental and the textual data are available, we can run MAXCCLUS to create subsets of the instances that behave in the same way during the microarray experiment.

\subsection{How MAXCCLUS works}

Figure 1.2 shows the MAXCCLUS clustering algorithm. First, MAXCCLUS uses the textual data to construct clusters of instances. Second, from these clusters MAXCCLUS selects the good clusters by using the experimental data. Third, MAXCCLUS attempts to generalise the good clusters producing the generalised clusters. Finally, from the generalised clusters MAXCCLUS attempts to create the cover clusters from which it constructs rules and characterisations. 


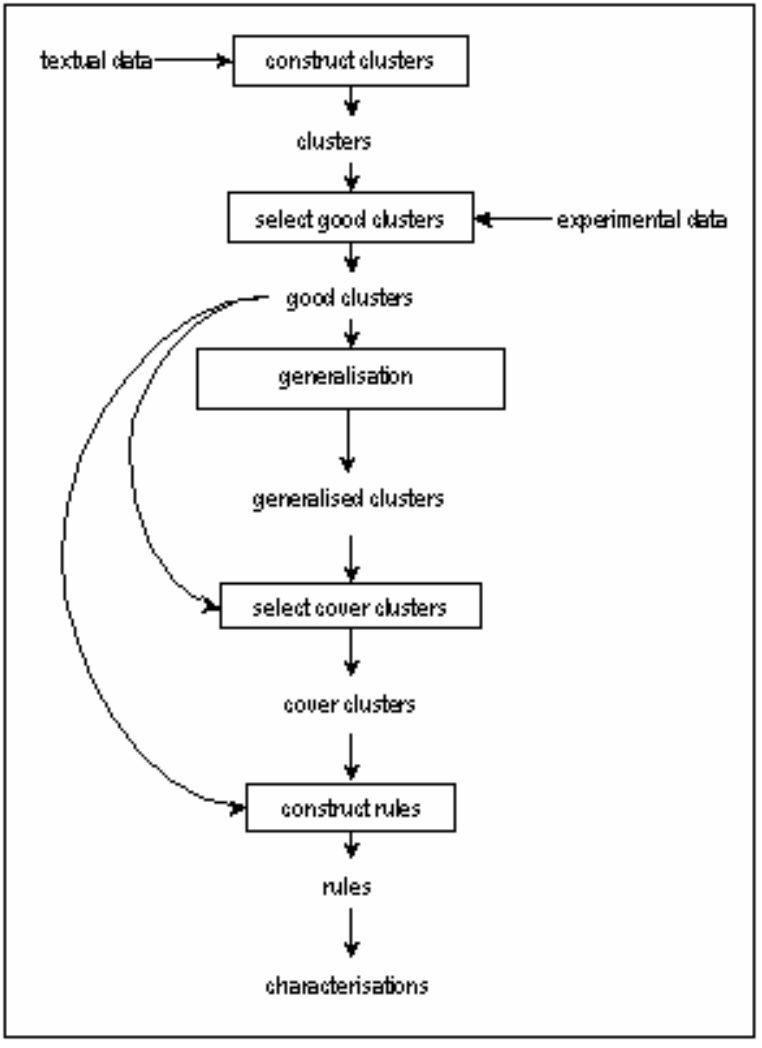

Figure 1.2: The MAXCCLUS clustering algorithm.

\subsubsection{Construct the clusters}

MAXCCLUS starts clustering the instances (genes) using the textual data by finding, for each pair of instances, the set of descriptors that are shared by these two instances. MAXCCLUS marks each pair of instances with the number of descriptors shared between them. Then for each marked pair of instances MAXCCLUS creates a cluster. Then MAXCCLUS adds to this cluster other instances that share the same descriptors with the cluster's instances.

The clusters that MAXCCLUS creates are determined only by the textual data while ignoring the instances' categories from the experimental data. Each of the constructed clusters has a set of descriptors (features) that is maximal in the sense that MAXCCLUS cannot add more descriptors to the cluster without 
excluding some instances from the cluster. Also each cluster is maximal in the sense that MAXCCLUS cannot add more instances to the cluster without reducing the number of descriptors that describe the cluster.

Each cluster MAXCCLUS creates can be viewed either as a set of instances or as a set of word (descriptors) that describe the cluster. We refer to the first view as the extensional view, and refer to the second as the intensional view.

Not all the clusters MAXCCLUS creates are relevant to the microarray experiment; and therefore not all of the clusters are meaningful. This is because, as mentioned above, the clusters that MAXCCLUS creates are determined only by the textual data while ignoring the instance categories from the experimental data. To obtain useful clusters, MAXCCLUS needs some criteria to determine which of the clusters that it constructs are relevant to the microarray experiment.

\subsubsection{Select good clusters}

MAXCCLUS considers a cluster to be a good cluster if it passes both of the two tests: the accuracy and the significance tests.

\section{The accuracy test}

One of the two conditions for a cluster to be considered as a good cluster is that a cluster needs to be accurate enough. Accuracy (purity) is a percentage measure of the number of instances in the cluster that have the same category (Up or Down). The accuracy is also reflects the accuracy of the rules that MAXCCLUS creates (in later step) to describe the cluster. The user that uses MAXCCLUS package determines the minimum accuracy. For example if the user determined that the accuracy should be $95 \%$, this means that each cluster must have $95 \%$, or more, of its instances having the same category for MAXCCLUS to consider this cluster as a good cluster. 


\section{The significance test}

The other condition for a cluster to be considered a good cluster is that an accurate cluster needs to be statistically significant. The significance test distinguishes between the accurate clusters that may result from a statistical chance, and the clusters that represent a real relationship between the descriptors (features) and the categories. MAXCCLUS runs a significance test on the clusters it creates to determine which clusters are statistically significant. The user of MAXCCLUS determines the minimum significance as a percentage (for example 95\%).

MAXCCLUS uses a permutation test. It does this test by repeatedly assigning categories (Ups or Downs) randomly to the instances. It then evaluates the purity of all the clusters, given these random assignments of categories. Any pure clusters it finds cannot represent significant clusters because the categories are random. There will be many small pure clusters, but fewer large pure clusters. It finds the minimum size cluster for which the probability of finding a pure cluster of this size is less than: 1-significance.

It then assigns the true categories to the instances and selects the pure clusters that are at least this minimum size. These clusters are presumed to be significant, and not the product of statistical chance, and are referred to as "good clusters" or "significant clusters".

After selecting good clusters, MAXCCLUS can go through one or more of three paths (viewing them from left to right in Figure 1.1):

The first path is that MAXCCLUS attempts to directly construct the rules that characterise the good clusters.

The second path is that MAXCCLUS attempts to select a subset of the good clusters that covers all the instances in the good clusters. It then constructs the rules that characterise these cover clusters. 
The third path is that MAXCCLUS attempts to generalise the good clusters to create generalised clusters; then selects a cover for the generalised clusters; after that constructs the rules that characterise the generalised cover clusters.

The following sections explain how MAXCCLUS constructs rules, selects cover clusters, and performs generalisation.

\subsubsection{Constructing rules}

MAXCCLUS attempts to construct rules that describe each cluster. MAXCCLUS uses the cluster's descriptors (words) to construct all the rules that describe all the instances (genes) that are in the cluster, and at the same time exclude all the instances that are not in the cluster.

MAXCCLUS constructs conjunctive rules (using "and" to combine words logically). For example, a rule that MAXCCLUS constructs to describe the instances of an Up regulated cluster of instances may look like this "Rule: protein, sequence, subunit, belong". This rule can be interpreted as: if an instance has all the words "protein", "sequence", "subunit", and "belong", then the instance is Up regulated. Saying it differently: all the instances in the cluster can be described by the conjunction of the words "protein", "sequence", "subunit", and "belong"; this conjunction of words excludes all the instances that are outside the cluster. MAXCCLUS does not construct disjunctive rules (using "or" to combine words logically).

\section{Words MAXCCLUS uses in constructing rules}

There are two sets of words that MAXCCLUS may use in constructing rules to describe a cluster, the Necessary and the Sufficient sets. 
The Necessary set of words contains the words that exist in every rule of the cluster. Each Necessary word is in the description of every gene in the cluster, but some genes outside the cluster share all but this word in the cluster's descriptors. These words are necessary to exclude these "near misses".

Sometimes the Necessary words are not enough to exclude some of the genes that are out the cluster, because some of these genes can have all of the Necessary words. MAXCCLUS finds another set of words, the Sufficient words. By using at least some of the Sufficient words in conjunction with the Necessary set of words, MAXCCLUS creates minimal rules of which each rule describes the instances in the cluster, but not true for the instances outside the, cluster even the ones that have all the Necessary words. The words from the Sufficient set of words are present in at least one but not all rules of the cluster.

For a rule to be minimal no word can be deleted from the rule without affecting the set of instances this rule describe. Deleting any word from a minimal rule allows the rule to also describe some instances from outside the cluster, which decreases the accuracy of the rule by describing instances that have different categories than the cluster's instances category.

\section{Word sets that MAXCCLUS uses in characterising a cluster}

Both the Necessary and the Sufficient sets of words characterise the instances of the cluster, but they are not the only ones. MAXCCLUS constructs two other sets of words to characterise the instances of a cluster, the Supplementary and the All but $X$ sets of words.

The Supplementary set of words contains the words that are not in any rule of the cluster, but are still true of all instances in the cluster. Since the Supplementary words are also true for some instances outside the cluster, these words do not help in distinguishing the cluster from other clusters. 
The All but $X$ set of words (also called frequent words) contains the words that are not in any rule of the cluster, but are still true of all but $X$ of the instances in the cluster. For example, a cluster may have 217 instances with characterisation that say "All but 2: hypothetical", which means that 215 of the cluster instances have the word "hypothetical" in their descriptor set, but the other 2 instances do not have this word.

Both the Supplementary and the All but $X$ sets of words are to help the user of MAXCCLUS (the scientist) by reflecting more of the characterisations of the instances in the cluster. These extra characterisations are not reflected in the rules.

\subsubsection{Generalisation}

MAXCCLUS attempts to generalise each of the good clusters by adding some instances from outside the cluster. The instances added share some of the same descriptors as the cluster's characterisation, but must have the same category as the cluster's category. Therefore, generalisation increases the accuracy of the cluster by increasing the number of correct instances. We call the resulting clusters the generalised clusters.

MAXCCLUS performs generalisation by constructing generalised rules that cover all the genes in the cluster and do not cover any incorrect instances outside the clusters, but may cover some additional correct instances. This small change to the rule constructions leads to a kind of generalisation that is simple and strict, however it is fast in execution and as good in covering instances as the more complex, and more time consuming generalisation introduced in Chapter 3. 


\subsubsection{Select cover clusters}

After constructing the good clusters (or the generalised clusters), MAXCCLUS attempts to select the cover clusters. Because the set of the good (or generalised) clusters may contain clusters that are overlapping and others that are subsumed by bigger clusters in the same set, MAXCCLUS attempts to select a minimal subset of the clusters that cover the same instances. We call this subset the cover clusters if it is the cover for the good clusters, or the generalised cover clusters if it is the cover clusters for the generalised clusters.

\subsection{How MAXCCLUS reports its clustering results}

After finishing clustering, MAXCCLUS reports its clustering results as an XML (Extensible Markup Language) file, which was originally rendered to $\mathrm{HTML}$ (HyperText Markup Language) file. Rendering was originally done by running a Java program on each of the XML files to create the respective HTML file. This approach is not flexible enough in case one needs to change how the HTML presents the data; furthermore this approach doubles the computer storage size needed to store only the XML files (because of having the HTML files too), which can be considerable when running MAXCCLUS on many data sets (some XML and HTML files can exceed 34MB in size). We modified the rendering by using only one small (7KB) XSL (Extensible Stylesheet Language) file to render, on the fly, any XML file created by MAXCCLUS, to a HTML file by using Microsoft Internet Explorer to open the XML file.

Figure 1.3 shows a snapshot of MAXCCLUS clustering results with Microsoft Internet Explorer using the XSL file. 


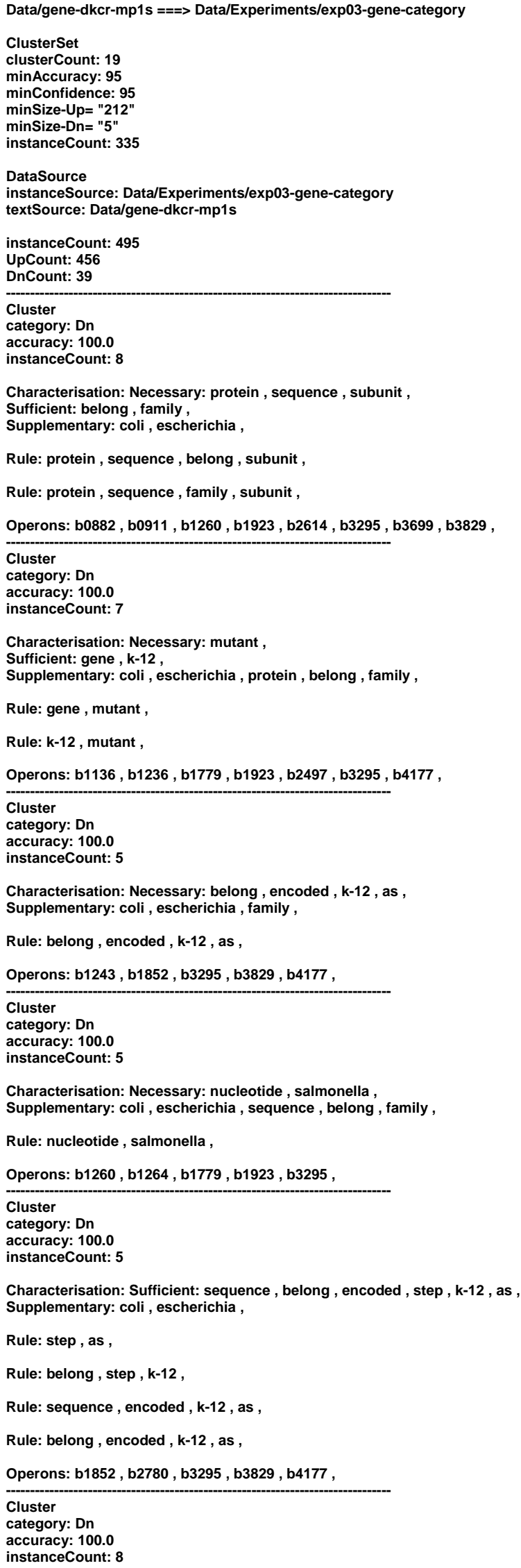

Figure 1.3: A snapshot of MAXCCLUS clustering results. 


\section{Chapter 2 Methodology}

This chapter describes the methodology we used to design the different clustering experiments and to analyse the results that MAXCCLUS produced from the experiments.

MAXCCLUS produces the results of its clustering and stores these results into $X M L$ files. Some of these $X M L$ files can exceed $34 M B$ in size. Browsing this amount of data and attempting to analyse it is a difficult job. Therefore we chose a methodology that can help us to simplify the results and enables us to compare different clustering experiments.

\subsection{The clustering experiments}

The project designed different clustering experiments to study the behaviour of MAXCCLUS. There are many control variables that one can change in a clustering experiment to create a new clustering experiment. We attempted to design the clustering experiments so that each one of them differs from the reference clustering experiment by one controlling variable. The reference clustering experiment is obtained by running MAXCCLUS using the experimental and textual data that is explained in Chapter 1. Comparing each one of the clustering experiments to the same reference clustering experiment (the normal clustering experiment) gives an understanding of the behaviour of MAXCCLUS and its limitations, and it helps identify improvements to MAXCCLUS, or the experimental or textual data that it uses. 
Figure 2.1 shows the reference clustering experiment, which we refer to as the before changes case, and the other clustering experiments, which we refer to as after changes case.

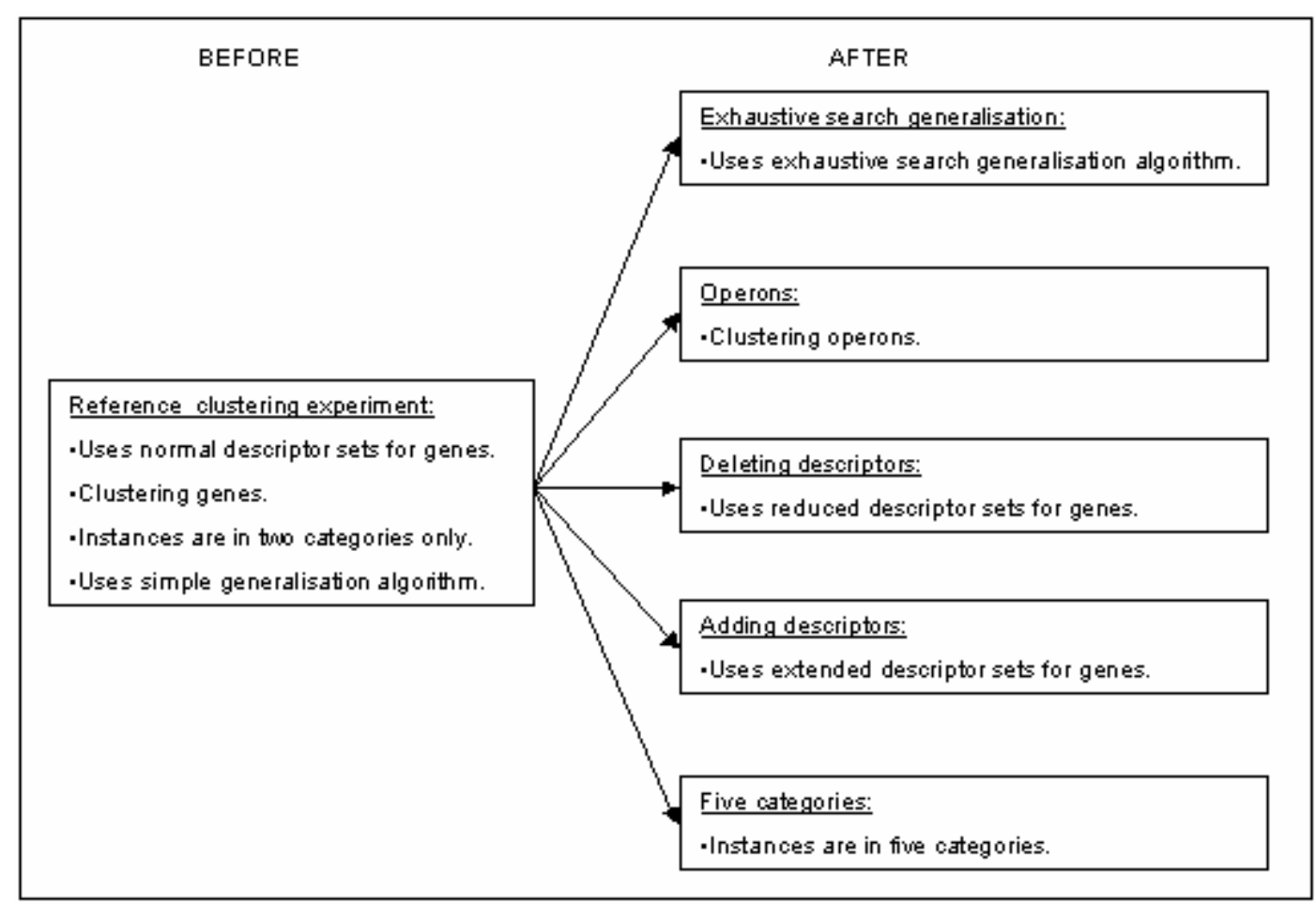

Figure 2. 1: The before and after changes clustering experiments.

Before any changes, in the reference clustering experiment, MAXCCLUS uses the normal descriptor sets for the genes (section 1.5), it attempts to cluster genes, the experimental data has instances (genes) that are in two categories only, and MAXCCLUS uses a simple generalisation algorithm when attempting to generalise the good clusters.

In the after changes case there are five clustering experiments. Each of these clustering experiments is based on the reference clustering experiment but with a change to one control variable. 
The first clustering experiment (Exhaustive search generalisation) is designed to study the behaviour of MAXCCLUS when MAXCCLUS uses an exhaustive search algorithm to generalise the good clusters, instead of the simple generalisation algorithm that it uses in the reference clustering experiment.

The second clustering experiment (Operons) is designed to study the behaviour of MAXCCLUS regarding the significance test. In this clustering experiment MAXCCLUS attempts to cluster operons instead of genes. Operons are logical groups of genes that should behave in the same way during the microarray experiment. Using operons instead of genes requires different textual data from the one the normal clustering experiment uses. The textual data for the operon clustering experiment has descriptor sets for operons created from the union of the descriptor sets of their genes.

The third and fourth experiments - Deleting descriptors and Adding descriptors - are designed to study the behaviour of MAXCCLUS when changes are made to the pre-processing of the textual data by changing the descriptor sets of the genes. Deleting descriptors uses reduced descriptor sets for the genes by deleting words from two different dictionaries. The second clustering experiment (Adding descriptors) uses extended descriptor sets for the genes by adding synonyms, hypernyms, or both synonyms and hypernyms of the existing descriptors.

The last clustering experiment (Five categories) is designed to study the behaviour of MAXCCLUS when it attempts to cluster instances that are in five categories instead of only two categories. In this clustering experiment, MAXCCLUS uses experimental data that includes all the genes (instances), not only the ones that are clearly Up or clearly Down regulated. 


\subsection{Comparing clustering experiments results}

To be able to study the behaviour of MAXCCLUS, we need to find a way to compare the results of using MAXCCLUS for each clustering experiment after having changes to the control variables, against the reference clustering experimental before changes to the control variables. We refer to the results of the reference clustering experiment with the word "before", and we refer to the results of each other clustering experiment with the word "after".

\section{What to compare}

MAXCCLUS reports its clustering results in XML files. The clustering results consist of some "objects". The objects can be clusters, instances, rules, or rules' words. We measured the clustering execution time separately because it is not reported in the XML files. Figure 2.2 list the maximum value of the objects count in an XML file to show the complexity of the comparison problem.

\begin{tabular}{|l|r|}
\hline \multicolumn{1}{|c|}{ Object } & Maximum count \\
\hline Instances & 980 \\
\hline Clusters & 1,789 \\
\hline Rules & 115,180 \\
\hline Rules' words & 306 \\
\hline
\end{tabular}

Figure 2.2: The maximum count for each object in an XML clustering results file.

The comparison is made between objects in the before and the after results. The result sections in the following chapters present the compared results as the relative change between the before and the after results. 
For each kind of object, the results are presented as the change to the total number of the objects, the number of retained objects (objects present in both before and after results), and the number of new objects. For $n$ microarray experiments data set, the calculations are as the following:

\section{Change\%}

For the percentage Change $\%, C_{i}$ is the percentage ratio of the number of after objects to the number of before objects in the $i$ th microarray experiment, calculated as:

$C_{i}=\frac{\mid \text { after }_{i} \mid}{\mid \text { before }_{i} \mid} \times 100$

\section{Retained\%}

For the percentage Retained\%, $R_{i}$ is the percentage ratio of the number of objects retained in the after clustering experiment, with respect to the number of objects in the before clustering experiment. The number of the retained objects is the number of objects that are in both the before and after results of the $i$ th microarray experiment. $R_{i}$ is calculated as:

$$
R_{i}=\frac{\mid \text { before }_{i} \bigcap \text { after }_{i} \mid}{\mid \text { before }_{i} \mid} \times 100
$$




\section{New\%}

For the percentage New\%, $N_{i}$ is the percentage ratio of the number of objects in the after results that were not in the before results of the $i$ th microarray experiment. $N_{i}$ is calculated as:

$N_{i}=\frac{\mid \text { after }_{i}-\text { before }_{i} \mid}{\mid \text { after }_{i} \mid} \times 100$

\section{Subsumed\%}

For the percentage Subsumed\%, $S_{i}$ is the percentage ratio of the number of objects in the before results that are subsumed by objects in the after results of the $i$ th microarray experiment. $S_{i}$ is calculated as:

$$
S_{i}=\frac{\mid \text { subsumed }_{i} \mid}{\mid \text { before }_{i} \mid} \times 100
$$

Where an object from before $_{i}$ is in subsumed $_{i}$ if there exists an object in after $_{i}$ that is strictly a generalisation of it.

Then we calculate the average, median, minimum value, maximum value, upper quartile, and lower quartile of $C_{i}, R_{i}, N_{i}$, and $S_{i}$ over $n$ microarray experiments data sets. We present the averages as histograms and other statistics as box and whisker plots [Williamson, D. 2002].

For each experiment, we calculate these measures for three sets of results from MAXCCLUS, the significant clusters (the good clusters, which are accurate and significant at the same time), the cover, and the generalised cover cluster sets, and present them side by side for comparison. 
Not all the clustering experiments use the same microarray data sets, because some of the clustering experiments could not handle some of the data sets for one of two reasons: first, not enough computer memory to continue the clustering task, and second, the clustering task takes a very long time, which forces us to kill the running process for these data sets. Figure 2.3 lists the clustering experiments and the microarray data sets used to study the clustering results.

\begin{tabular}{|l|c|l|}
\hline $\begin{array}{l}\text { Clustering } \\
\text { experiment }\end{array}$ & $\begin{array}{c}\text { Number } \\
\text { of data } \\
\text { sets }\end{array}$ & \multicolumn{1}{c|}{ Data sets } \\
\hline $\begin{array}{l}\text { Exhaustive } \\
\text { search } \\
\text { generalisation }\end{array}$ & 23 & $\begin{array}{l}3,6,7,8,10,11,12,13,16,18,20,21,22,23,24,25,27,28, \\
29,30,35,36,40\end{array}$ \\
\hline Operons & 20 & $\begin{array}{l}6,7,10,11,12,16,18,20,21,22,23,24,25,27,28,29,30,35, \\
36,40\end{array}$ \\
\hline $\begin{array}{l}\text { Deleting } \\
\text { descriptors }\end{array}$ & 23 & $\begin{array}{l}3,6,7,8,10,11,12,13,16,18,20,21,22,23,24,25,27,28, \\
29,30,35,36,40\end{array}$ \\
\hline $\begin{array}{l}\text { Adding } \\
\text { Descriptors }\end{array}$ & 23 & $\begin{array}{l}3,6,7,8,10,11,12,13,16,18,20,21,22,23,24,25,27,28, \\
29,30,35,36,40\end{array}$ \\
\hline $\begin{array}{l}\text { Five } \\
\text { categories }\end{array}$ & 19 & $\begin{array}{l}3,6,7,10,11,12,13,16,18,20,21,22,23,24,25,27,28,29, \\
30\end{array}$ \\
\hline
\end{tabular}

Figure 2.3: The clustering experiments and the microarray data sets used in studying the clustering results. 


\subsection{How much of the data can MAXCCLUS describe}

Figure 2.4 shows the number of instances described originally by MAXCCLUS when running the reference clustering experiment on 38 microarray experimental data sets. On average, MAXCCLUS can describe about $37 \%$ of the instances in the data sets. The distributions show that there is a wide variation across the data sets: from about $5 \%$ to about $79 \%$.

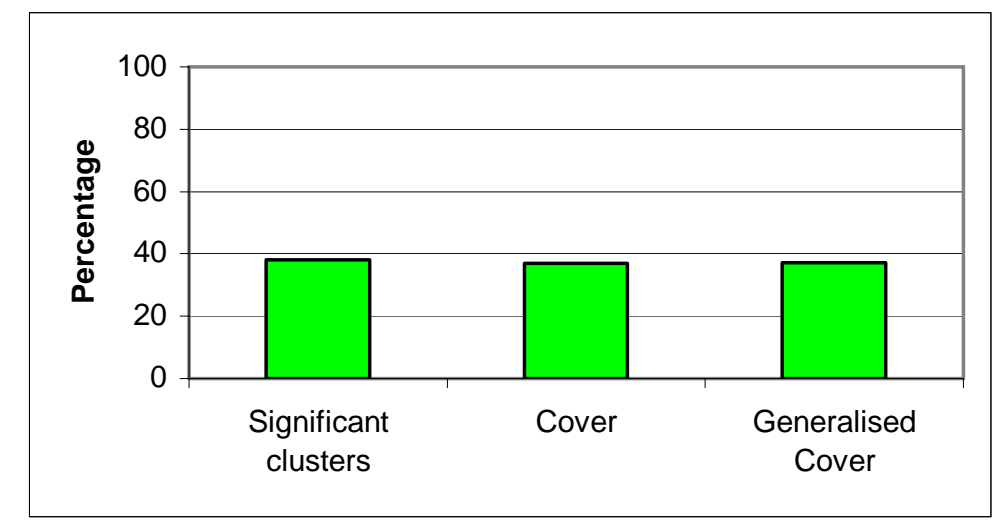

a: Average

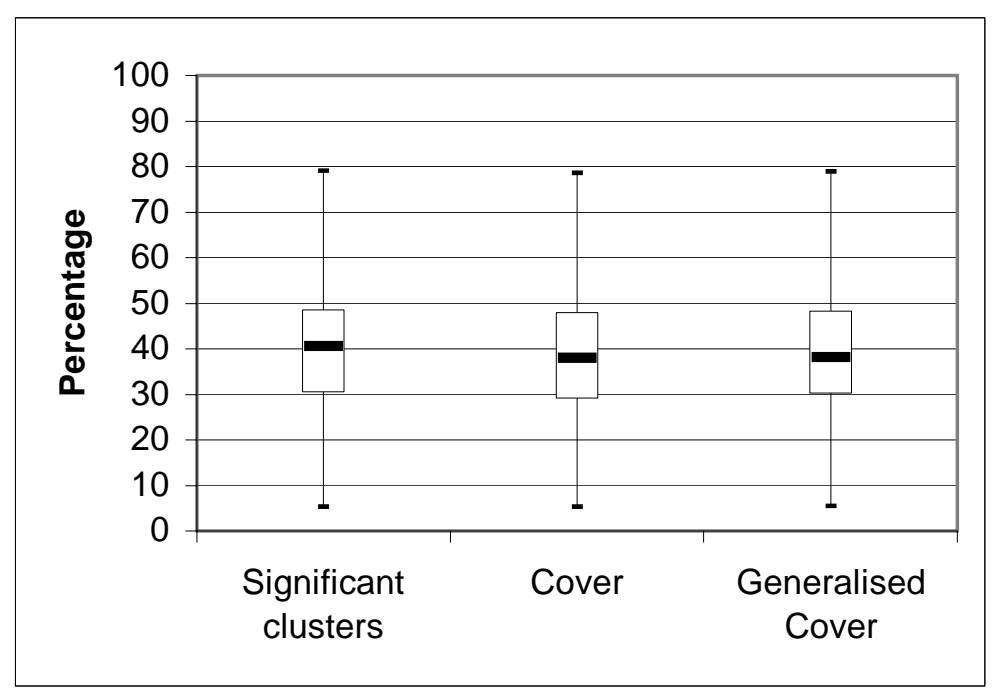

b: Distributions

Figure 2.4: Number of instances described by running the reference clustering experiment. 


\section{Chapter 3}

\section{Exhaustive Search Generalisation}

This chapter describes a clustering experiment designed to study MAXCCLUS's generalisation by introducing an exhaustive search generalisation clustering experiment and comparing its results with MAXCCLUS's simple generalisation used in the reference clustering experiment. The surprising result of this chapter is to show that MAXCCLUS's simple generalisation does very well compared with the more complex and more expensive exhaustive search generalisation, by explaining all the instances with much less execution time.

In the reference clustering experiment, MAXCCLUS uses a simple generalisation that attempts to add, to the cluster that is attempting to generalise, other instances that have the same category as the cluster. It does this using greedy algorithm that effectively drops words from the description of the cluster without including other instances that have different category from the cluster. We thought that having an exhaustive search generalisation would make MAXCCLUS able to generalise a cluster better than the simple greedy generalisation, by using a powerful search an by allowing some instances that have a different category from the cluster's category to be added to the cluster, as long as the accuracy is not sacrificed. 


\subsection{Data source}

Both versions of MAXCCLUS use the same data as their input, the experimental data and the textual data as described in chapter 1.

\subsection{Comparison between the two versions of MAXCCLUS}

When generalising in the reference clustering experiment (see chapter 1), MAXCCLUS performs a simple generalisation. It only adds correct instances (instances that have the same category as the original cluster's category) and it only generalises by dropping descriptors. Therefore there is a limit for MAXCCLUS's simple generalisation. There is a possibility of getting more general rules and clusters by using more powerful algorithm than MAXCCLUS. MAXCCLUS needs a generalisation that can search the space of the cluster's characterisation words exhaustively, looking through all the possible combinations, and can accept incorrect instances (instances that have categories different than the original cluster's category) providing that the cluster's accuracy is not sacrificed.

\subsubsection{MAXCCLUS's two versions}

Figure 3.1 shows two versions of MAXCCLUS. Both versions are the same except for the generalisation part: one uses the simple generalisation and the other uses an exhaustive search generalisation. Both versions start by constructing clusters using the textual data annotated with each instance (gene). From these constructed clusters, the two versions select the good clusters (the significant accurate clusters) using the microarray experimental data. Then both versions attempt to generalise the good clusters, either by using the simple generalisation, or by using the exhaustive search 
generalisation. From these generalised clusters both versions select the cover clusters to get the generalised cover clusters. From these generalised cover clusters, both versions construct the rules that characterise the clusters.

The following sections describe the exhaustive search generalisation method.

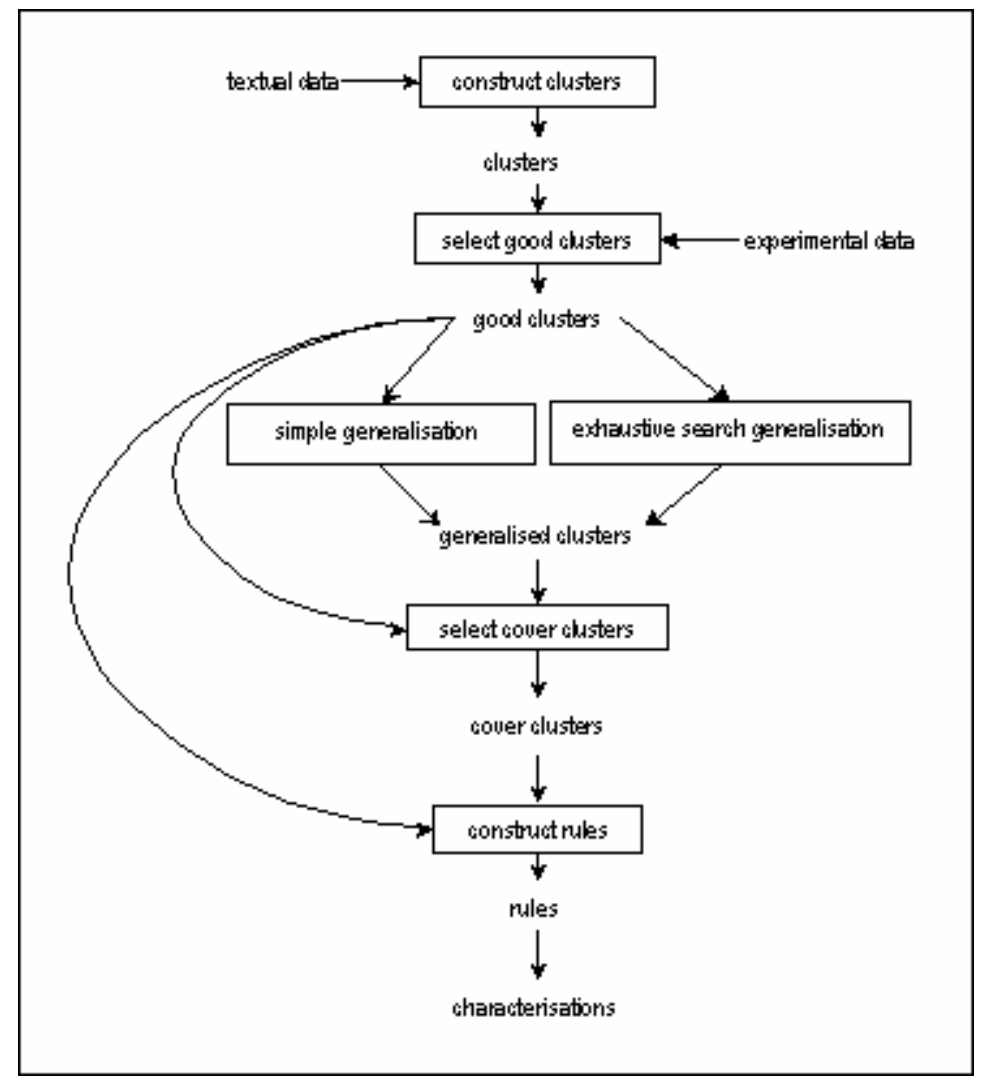

Figure 3.1: The two versions of MAXCCLUS. One uses the simple generalisation (the reference clustering experiment) while the other uses the exhaustive search generalisation (this chapters clustering experiment). 


\subsubsection{Simple generalisation - algorithm}

The simple generalisation that MAXCCLUS uses is based on only dropping words from the rules that describe the cluster, so that the new generalised rule will cover more instances. This generalisation does not allow negative instances (instances that have different category than the cluster's category) to be covered by the generalised rule.

\subsubsection{Exhaustive search generalisation}

The input to the exhaustive generalisation algorithm is a good cluster (which is a significant and pure cluster). The output is the best generalisation of this cluster. The algorithm searches for the best generalised rule, by attempting to generalise one of the cluster's rules (each rule consists of words that describe the cluster's instances). The best generalised rule for a cluster is a rule that has the same category as the cluster and describes more instances than any other rule of the cluster, subject to a constraint on accuracy (described later in section 3.2.3.2). The best generalised rule for the cluster could be one of the original rules of the cluster, or it could be a new rule that also describes the cluster. From the best generalised rule (the best generalisation of the cluster), MAXCCLUS can construct a new cluster, which is bigger than the original cluster (describes more instances than the original cluster).

The exhaustive generalisation algorithm creates a set of words that are in the characterisation of the cluster. Words contains all the common words and all the frequent words of the cluster. The common words of a cluster are the words that are represented in all the instances of the cluster; the frequent words of a cluster are the words that are represented in most of the instances of the cluster. The exhaustive generalisation algorithm (figure 3.2) searches exhaustively by creating all combinations (all subsets) of the set of words that are in the characterisation of the cluster.

This project implemented two versions of the exhaustive generalisation algorithm, the less restricted version and the more restricted version. They are 
similar to each other; they only differ in line 04 of the algorithm (figure 3.2). The more restricted version includes the "a superset of" constraint, and the less restricted version does not.

The exhaustive generalisation algorithm starts by choosing the rule that has fewest words from the rules that describe the cluster. Initially this rule becomes the best rule. From each combination (subset) of words, except the empty set $\varnothing$, and combinations that are equal to (for the less restricted version), or supersets of (for the more restricted version) the original rules of the cluster, this algorithm creates a new rule. This new rule has the same category of the cluster. The algorithm evaluates the new rule against the best rule by using the IsBetterThanOrEqualTo method (described in the next section).

If the new rule is evaluated as better than or equal to the best rule, and if the new rule covers more correct instances than the best rule, or the new rule has fewer words than the best rule, then the new rule becomes the best rule (Note: "correct instances" are the instances that have the same category as the rule). Choosing the rule that has fewer words results in a simpler rule to describe the cluster. When the exhaustive search finishes, this algorithm returns the best rule.

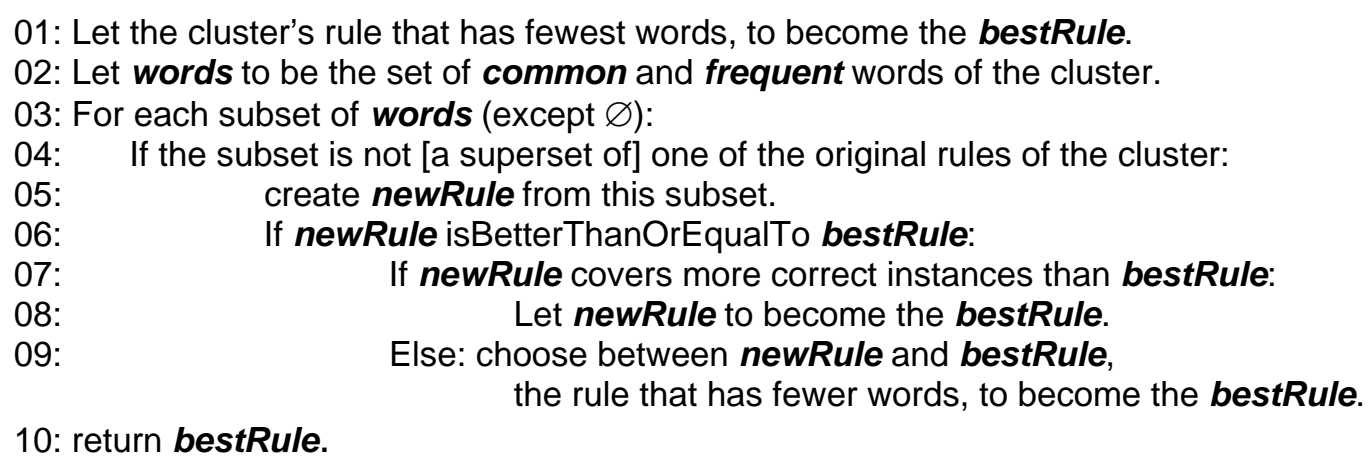

Figure 3.2: The exhaustive generalisation algorithm 


\subsubsection{IsBetterThanOrEqualTo - three evaluating criteria}

The input to this method is two rules: the best rule found so far, and the new rule that the exhaustive generalisation algorithm trying to evaluate with respect to the current best rule. IsBetterThanOrEqualTo returns true if the new rule is better than or equal to the best rule. The project implements two versions of the IsBetterThanOrEqualTo method, depending on two approaches for evaluating accuracy.

\subsubsection{Two criteria: Accuracy and Minimum-accuracy}

The IsBetterThanOrEqualTo method has three conditions that must be satisfied to return the value true.

The first two conditions are (see figures 3.3 and 3.4):

1. The number of newRule's correctly covered instances is greater than or equal to the number of bestRule's correctly covered instances.

2. The correctly shared instances between newRule and firstRule are greater than or equal to a percentage value (the implementation uses $70 \%)$.

There are two forms of the third condition referred to as accuracy and minimum-accuracy.

Accuracy is the accuracy of the rule and is calculated as:

$$
A=\frac{C}{I}
$$

Where $A$ is the accuracy, $C$ is the number of correct instances the rule covers, and $I$ is the number of all instances the rule covers. 
Minimum-accuracy is the accuracy specified by the user of MAXCCLUS. It determines the minimum accuracy for a cluster that MAXCCLUS should handle.

For the accuracy form, the third condition is (figure 3.3):

3. newRule's accuracy is greater than or equal to bestRule's accuracy.

For the minimum-accuracy form, the third condition is (figure 3.4):

3. newRule's accuracy is greater than or equal to the minimum accuracy for the clusters (usually minimum accuracy is 95\% pure). The user of MAXCCLUS specifies the value of the minimum-accuracy.

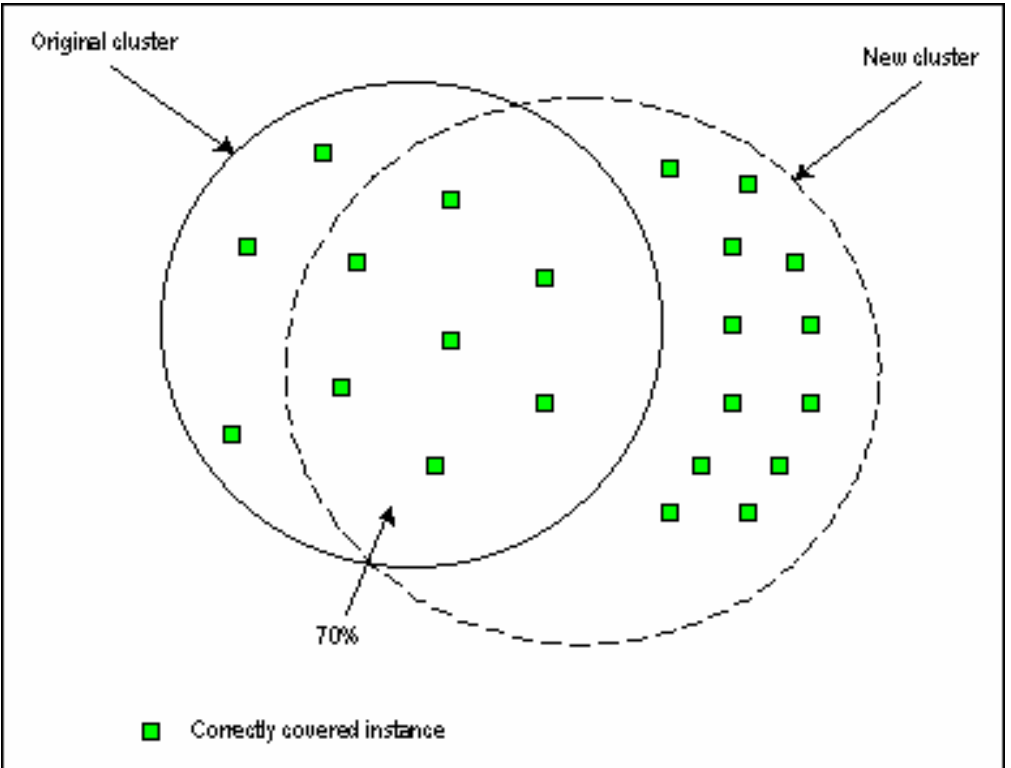

Figure 3.3: A new cluster, that is created from the best generalised rule, must cover at least $70 \%$ of the correctly covered instances of the original cluster. The new cluster's accuracy is equal to the original cluster's accuracy (in this example both accuracies are 100\%). 


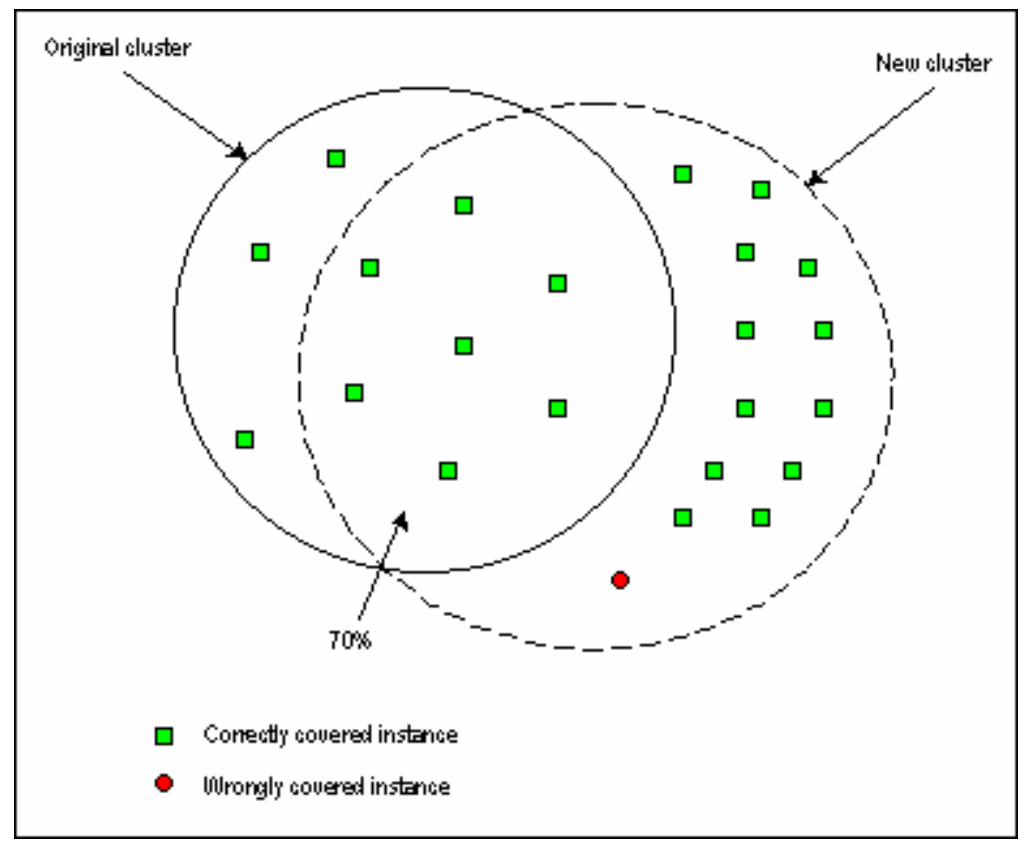

Figure 3.4: A new cluster, that is created from the best generalised rule, covers more correct instances than the original cluster. The new cluster must also cover at least $70 \%$ of the correctly covered instances of the original cluster. Also the new cluster's accuracy must be greater than or equal to minimum accuracy. Supposing that the minimum accuracy is 95\%, in this example the new cluster's accuracy is equal to the minimum accuracy.

\subsection{Experimental results}

Because the two versions of MAXCCLUS are identical except for the generalisation part, both versions have the same significant clusters and the same cover clusters. Therefore the results section compares only the generalised cover clusters of the two versions.

The results shown in the figures below are for running MAXCCLUS on 23 of the microarray experimental data sets (data set: $3,6,7,8,10,11,12,13,16$, $18,20,21,22,23,24,25,27,28,29,30,35,36$, and 40), using exhaustive search generalisation instead of the simple generalisation. Figures 3.5 to 3.14 show the results for the number of clusters, the number of rules, the number of words in the rules, the number of words per rule, and the execution time. 
We will refer in the remaining of the chapter to the results using accuracy as $\boldsymbol{A C}$, and using minimum accuracy as $\boldsymbol{M A}$.

\section{The clusters}

Figures 3.5 to 3.7 show the changes to the number of clusters when MAXCCLUS uses the exhaustive search generalisation instead of the simple generalisation. The figures show the changes to the total number of clusters, the number of retained clusters, and the number of new clusters.

On average, the number of clusters when MAXCCLUS uses the exhaustive search generalisation is about $5 \%$ more than when MAXCCLUS uses the simple generalisation (figure 3.5).

When MAXCCLUS uses the exhaustive search generalisation, on average, $45 \%$ of the clusters are retained from the clusters found when MAXCCLUS uses the simple generalisation (figure 3.6).

When MAXCCLUS uses the exhaustive search generalisation, on average, about $56 \%$ of the clusters found are different from the clusters found when MAXCCLUS uses the simple generalisation (figure 3.7).

On average, almost no clusters found from clustering when MAXCCLUS uses the simple generalisation, are subsumed by new clusters found from clustering when MAXCCLUS uses the exhaustive search generalisation. 


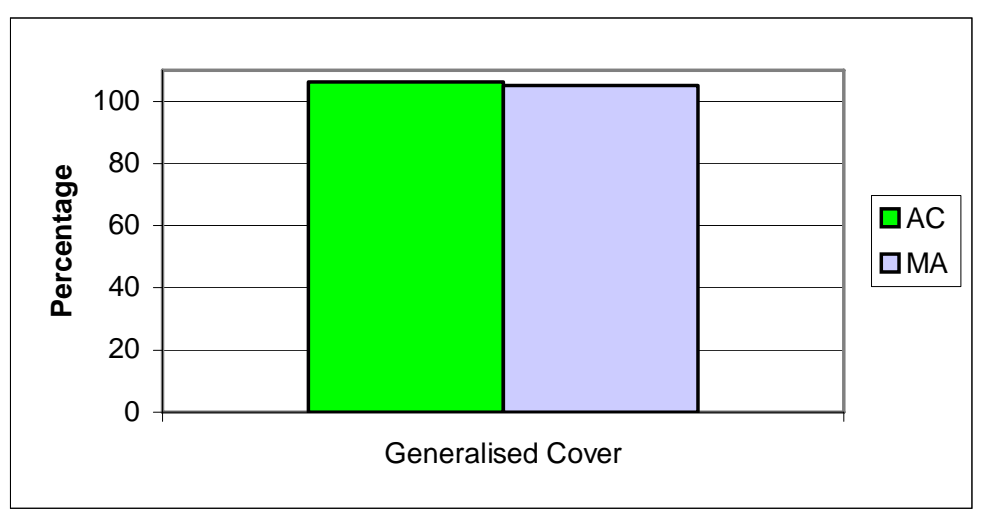

Figure 3.5: Average change to the total number of clusters

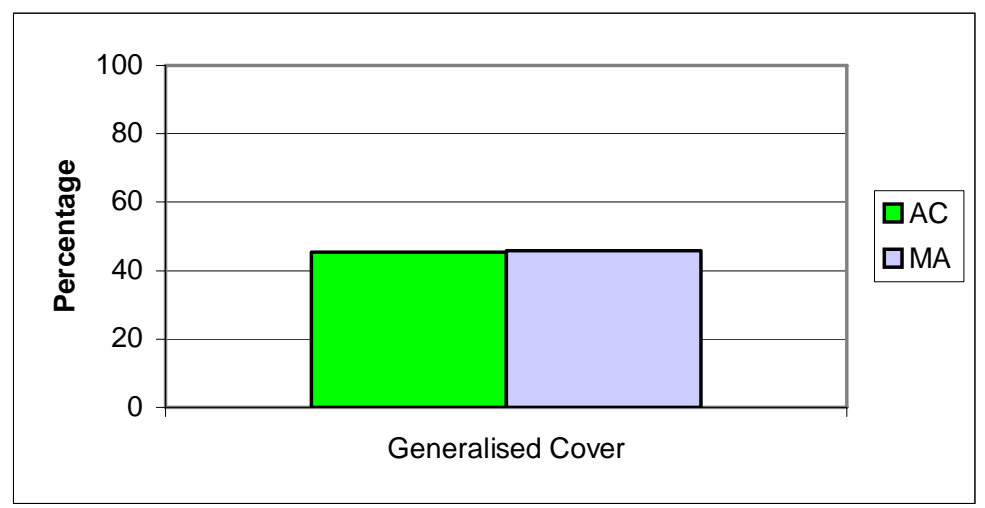

Figure 3.6: Average number of retained clusters

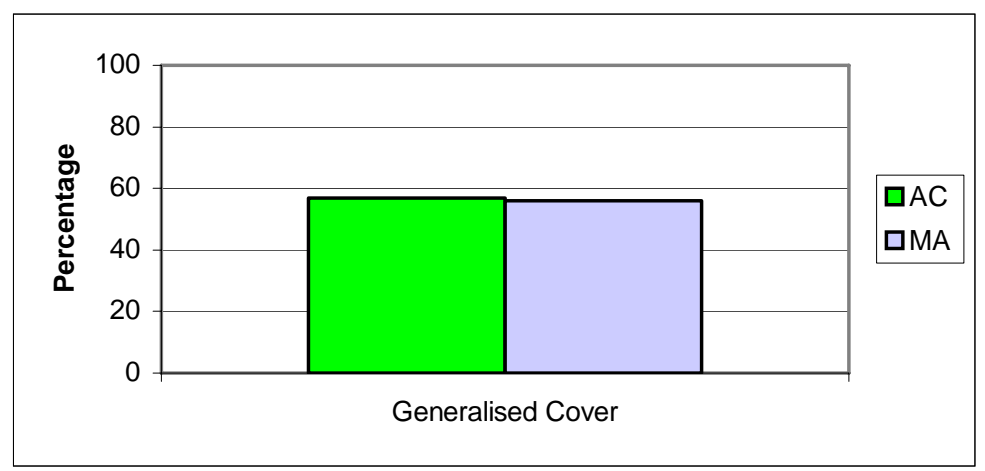

Figure 3.7: Average number of new clusters 


\section{The instances}

When MAXCCLUS uses the exhaustive search generalisation it describes the same instances as it does when uses the simple generalisation.

\section{The rules}

Figures 3.8 to 3.10 show the changes to the number of rules when MAXCCLUS uses the exhaustive search generalisation instead of the simple generalisation. The figures show the changes to the total number of rules, the number of retained rules, and the number of new rules.

On average, the number of rules when MAXCCLUS uses the exhaustive search generalisation is about $65 \%$ more that when MAXCCLUS uses the simple generalisation (figure 3.8), but the Accuracy version of the algorithm (AC) has a somewhat wider range.

When MAXCCLUS uses the exhaustive search generalisation, about $81 \%$ of the rules are retained from the rules found when MAXCCLUS uses the simple generalisation (figure 3.9).

When MAXCCLUS uses the exhaustive search generalisation, on average, $46 \%$ of the rules are different from the rules found when MAXCCLUS uses the simple generalisation (figure 3.10).

On average, almost no rules found from clustering when MAXCCLUS uses the simple generalisation, are subsumed by new rules found from clustering when MAXCCLUS uses the exhaustive search generalisation. 


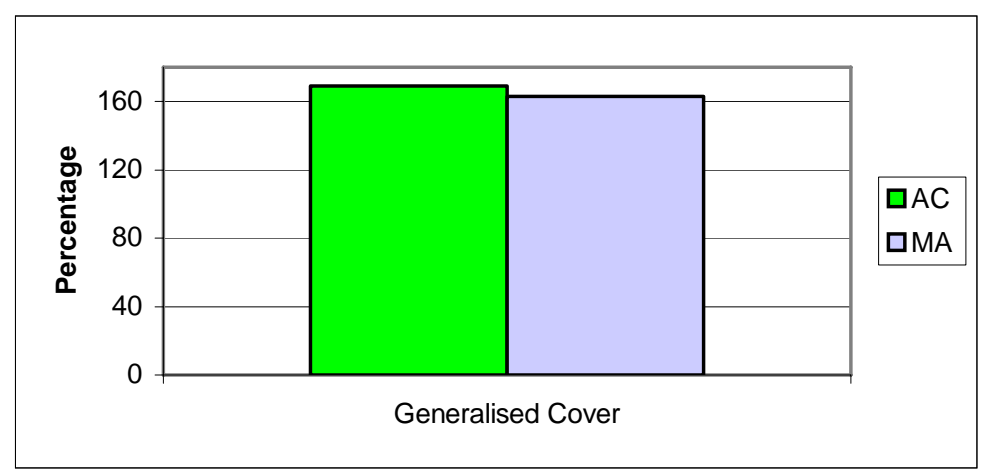

a: Average

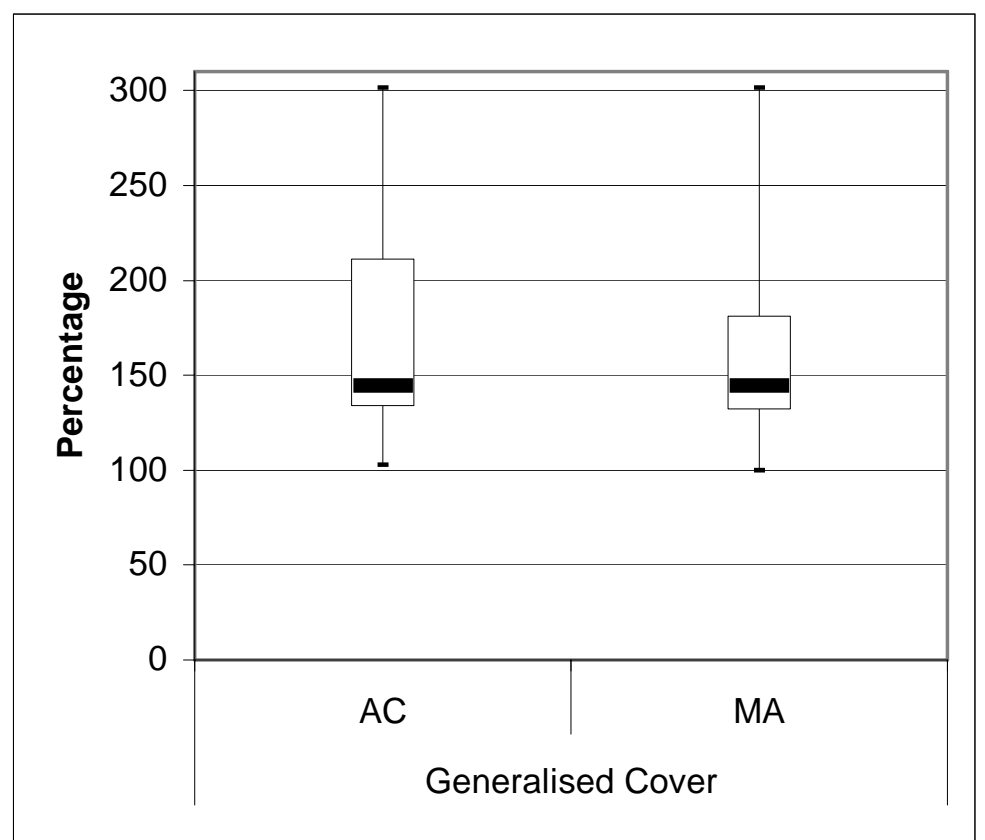

b: Distributions

Figure 3.8: Change to the total number of rules

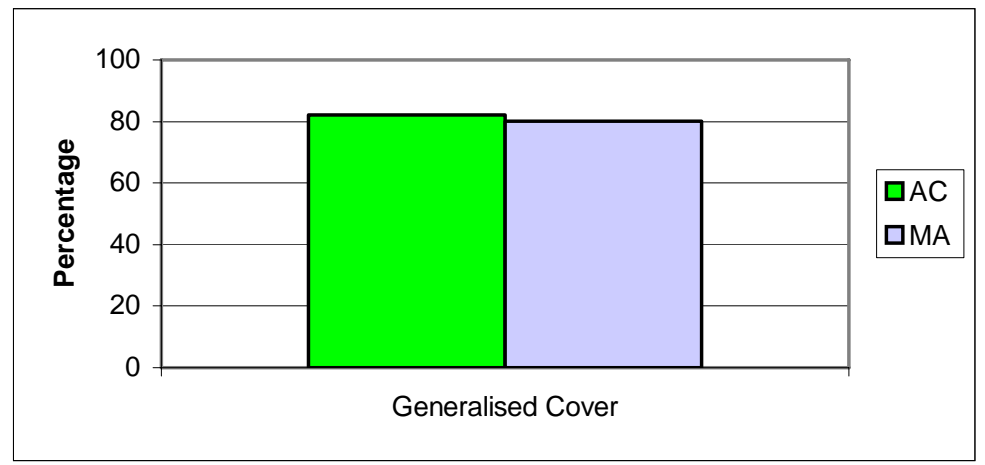

Figure 3.9: Average number of retained rules 


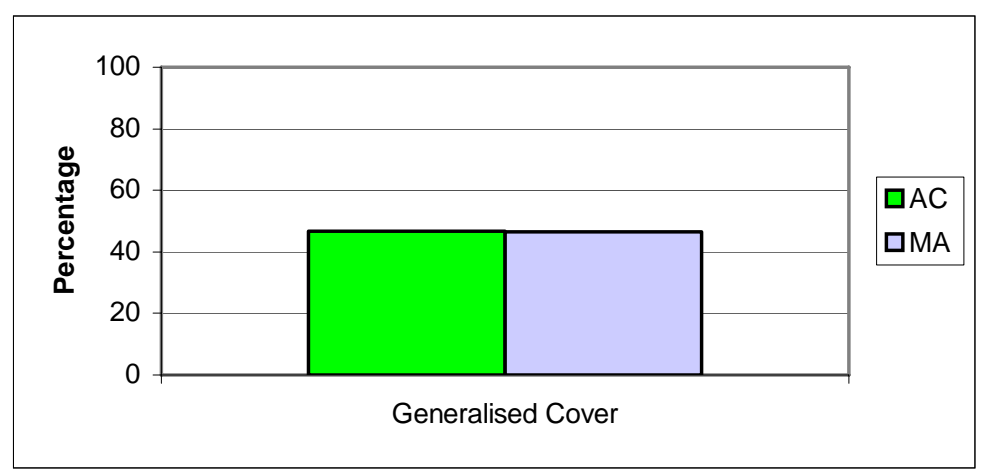

Figure 3.10: Average number of new rules

\section{The rules' words}

Figures 3.11 to 3.13 show the changes to the number of rules' words when MAXCCLUS uses the exhaustive search generalisation instead of the simple generalisation. The figures show the changes to the total number of rules' words, the number of retained rules' words, and the number of new rules' words.

On average, the number of rules' words when MAXCCLUS uses the exhaustive search generalisation is about $13 \%$ more than that when it uses the simple generalisation (figure 3.11).

When MAXCCLUS uses the exhaustive search generalisation, about $96 \%$ of the rules' words are retained from that found when MAXCCLUS uses the simple generalisation (figure 3.12).

When MAXCCLUS uses exhaustive search generalisation, on average, about $15 \%$ of the rules' words are different from the rules' words found when it uses the simple generalisation (figure 3.13). 


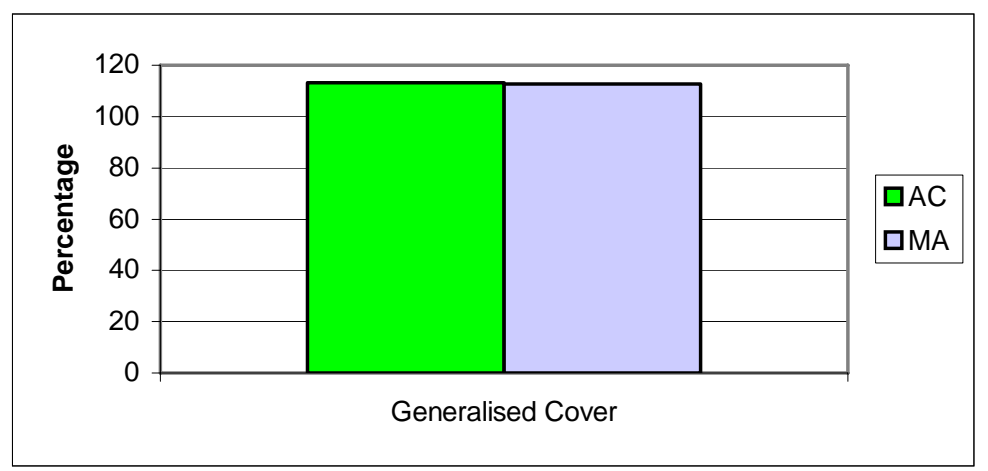

Figure 3.11: Average change to the total number of rules' words

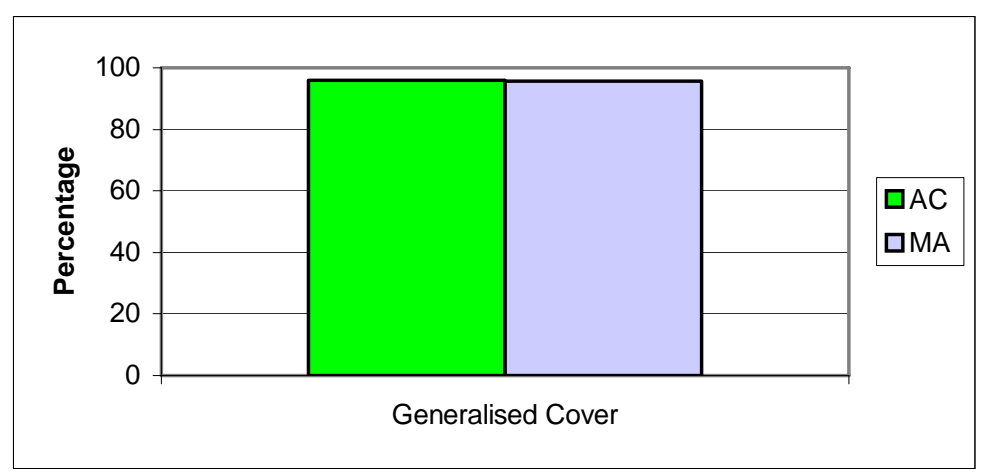

Figure 3.12: Average number of retained rules' words

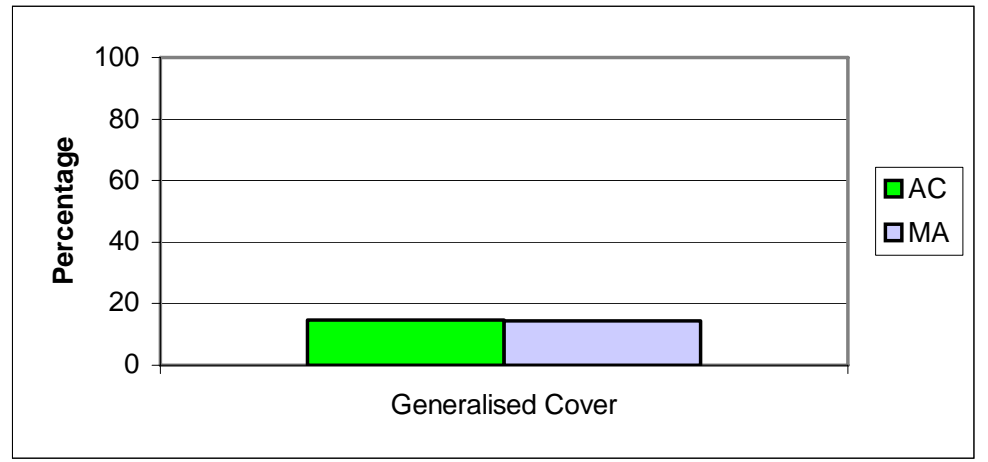

Figure 3.13: Average number of new rules' words 


\section{The words per rule}

Figure 3.14 shows the changes to the total number of words per rule when MAXCCLUS uses the exhaustive search generalisation instead of the simple generalisation.

On average, the number of words per rule when MAXCCLUS uses the exhaustive search generalisation is almost the same (only about $2 \%$ more) as that when MAXCCLUS uses the simple generalisation

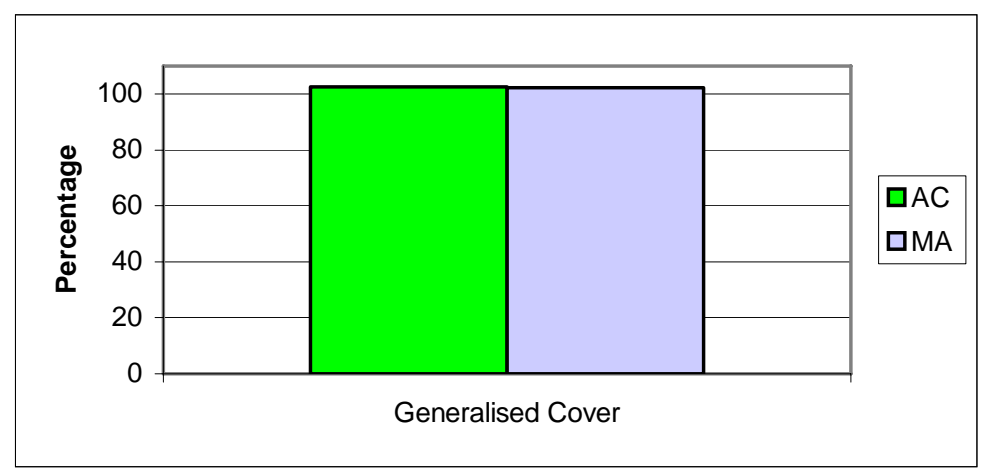

Figure 3.14: Average change to the number of words per rule

\section{The execution time}

Figure 3.15 shows the changes to the clustering execution time when MAXCCLUS uses the exhaustive search generalisation instead of the simple generalisation.

When MAXCCLUS uses the exhaustive search generalisation using AC or $M A$, on average, the execution time is much more than that when MAXCCLUS uses the simple generalisation. On average, the execution time, when clustering using $A C$, is slightly more than that when using MA; about 7.9 times longer when using AC, but only about 7.6 times longer when using MA, compared with the execution time when MAXCCLUS uses the simple generalisation. 


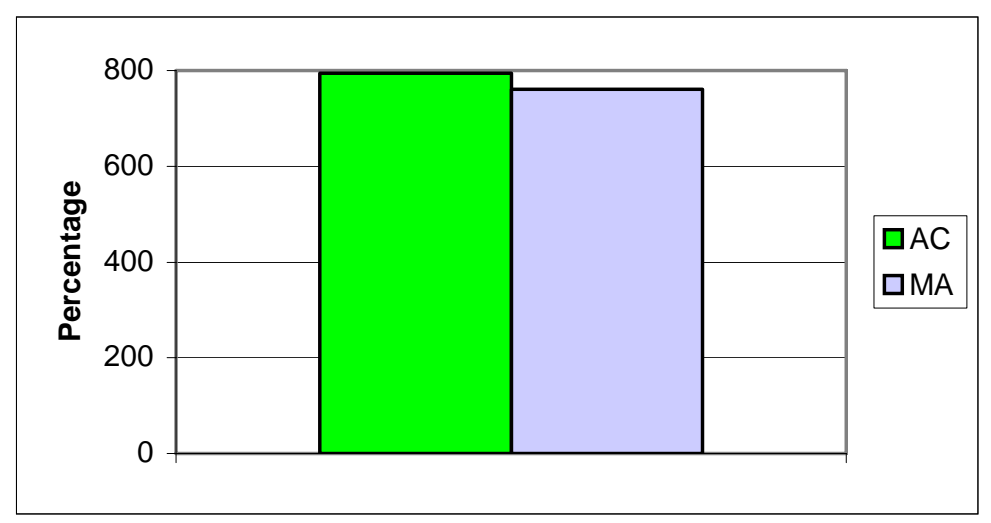

Figure 3.15: Average change to the clustering execution time

\subsection{Analysis and discussion}

The following sections analyse and discuss the results shown in the previos section.

The MAXCCLUS clustering algorithm has a simpler and faster algorithm for generalisation, compared with more complex and much slower exhaustive search algorithm for generalisation of the modified clustering algorithm. We had expected that the exhaustive algorithm would give noticeably better clusters and rules. However, the results show that MAXCCLUS, using its simple generalisation algorithm, behaved very well with most of the microarray experimental data sets.

The modified clustering algorithm created new clusters, from the best rules, and then it added these new clusters to the original clusters giving a bigger cluster set. Creating and adding the new clusters to the original cluster set, had little effect on the characterisation behaviour of MAXCCLUS, as the results show. There was little change in the number of clusters in the generalised cover and no change to the instances. Therefore the original MAXCCLUS generalisation algorithm, with its simplicity and speed, proved to be preferable to the more complex and more time consuming modified 
clustering algorithm using either of the two different criteria accuracy and minimum accuracy.

Although the exhaustive search generalisation allowed adding negative instances that have different category from the cluster, the number of instances did not increase. This could be because of the high user accuracy value (95\%), which prevents adding lots of negative instances to keep the high purity of the clusters. We believe that if the user uses low accuracy value (say 70\%) then more negative instances would be added to the clusters, but then the clusters would not be interesting because of the low accuracy value.

\subsection{Conclusion}

Using simple generalisation approach for MAXCCLUS is better, for the microarray data sets used for clustering experiments, than using an exhaustive search generalisation. The simple generalisation can describe all the instances that the exhaustive search generalisation can describe, and it is much faster than the exhaustive search generalisation, at least for the microarray data sets that MAXCCLUS used in this project.

In the future work we will experiment with other data sets (something that was not available during the period of this project) by running this chapter's experiment and the reference experiment and compare their results as we did in this chapter. We expect that the simple generalisation will do as well as the exhaustive search generalisation in describing instances and it will be faster, as it did with the microarray data sets that used in this project.

\subsection{Recommendations}

For the reasons mentioned in the conclusion section, we recommend that MAXCCLUS keeps its simple generalisation and there is no need to replace it with an exhaustive search generalisation, at least for the microarray data sets that MAXCCLUS used in this project with the given high user accuracy. 


\section{Chapter 4}

\section{Operons}

This chapter explores running MAXCCLUS to cluster operons instead of genes. An operon is a group of genes that behave in the same way regarding regulation. So we expect that within a microarray experiment, a set of genes that belong to an operon will respond to physical and/or chemical effects in the same way. This means an operon is already a cluster of genes, even before using a clustering algorithm (Figure 4.1).

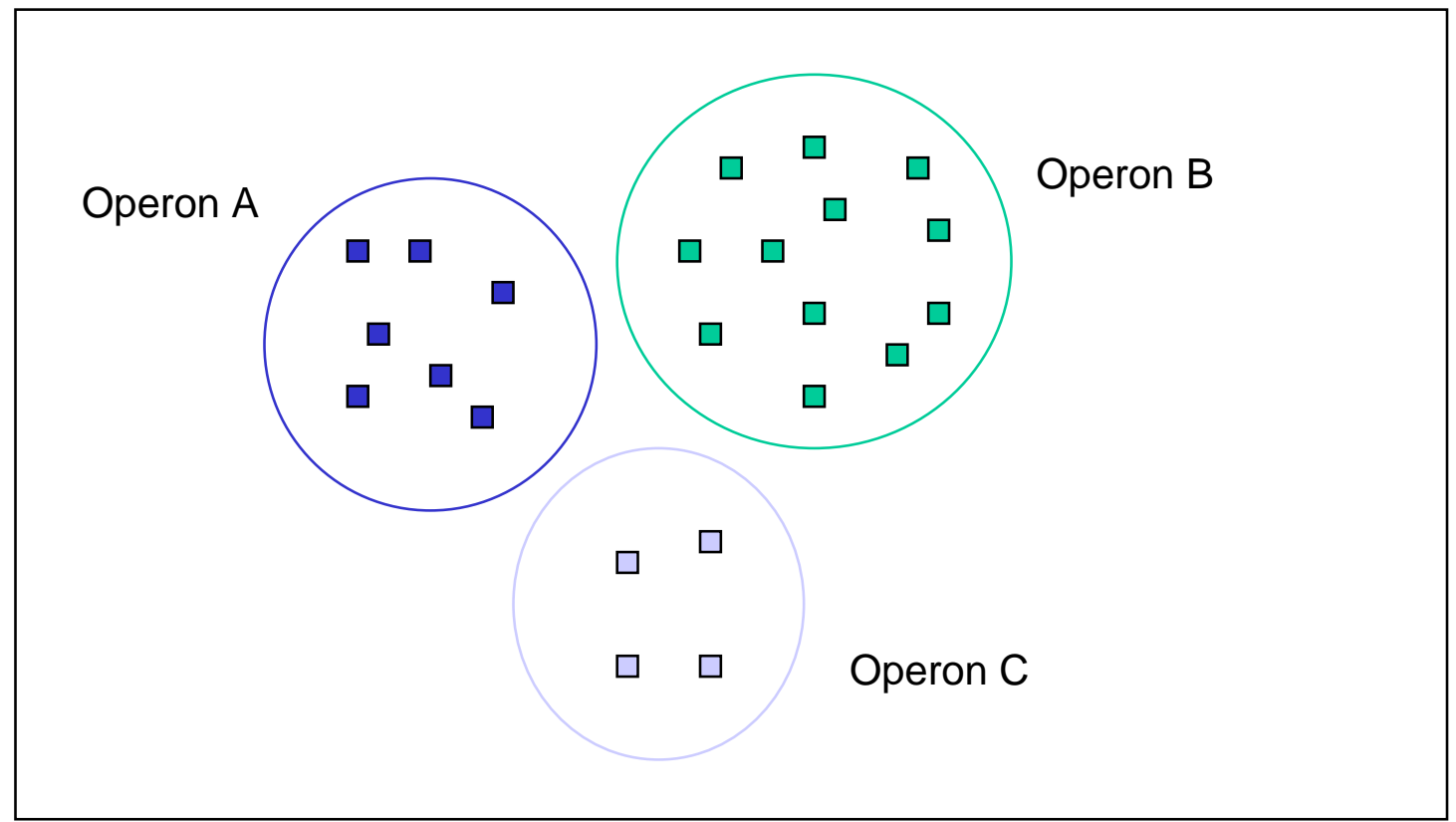

Figure 4.1: An operon is a group of genes that behave in the same way. 
By clustering operons instead of genes we expect to reduce the number of clusters produced by MAXCCLUS, and we will find out if the clusters found by clustering genes were scientifically interesting, or if they were clusters of genes that represent operons.

When clustering genes, MAXCCLUS performs a significance test on each cluster it finds. The question "Is this cluster big enough to be significant?" is based on an assumption that all the genes in the cluster were independent of each other. When performing the significance test, MAXCCLUS does not consider that some of the genes belong to operons, and therefore ignores their dependency on each other. When clustering operons, we are questioning the significance of the clusters found by MAXCCLUS when clustering genes.

Because there are not many big operons (figure 4.2), it is not the case that MAXCCLUS could find lots of gene clusters that are operons. But MAXCCLUS can find gene clusters that contain a number of genes that belong to the same operon.

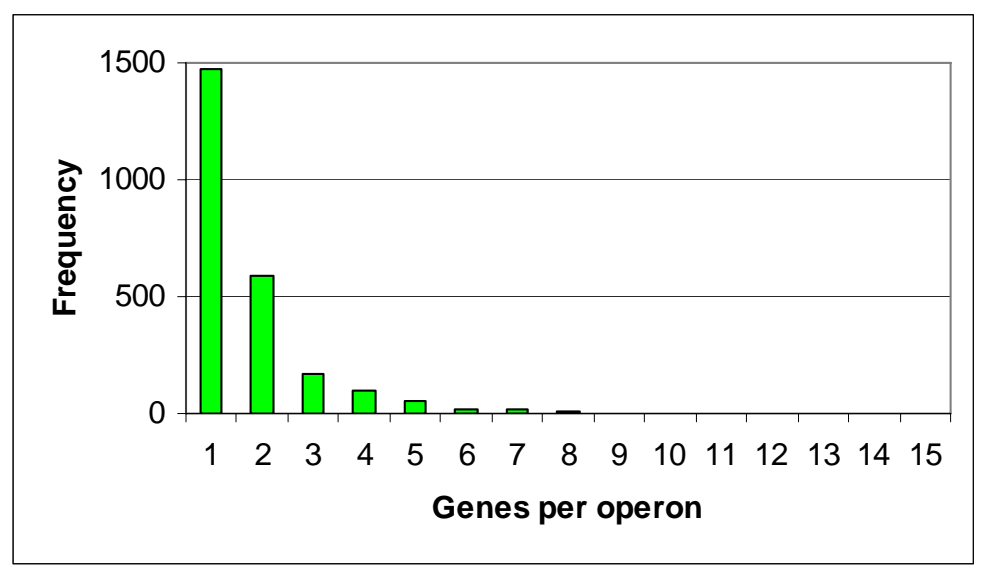

Figure 4.2: The frequency of genes per operon. 
Having genes that belong to the same operon, in a cluster found by MAXCCLUS, changes the calculations of the significance test.

\subsection{Example}

For example, suppose, when clustering genes, MAXCCLUS finds a cluster of 10 genes (figure 4.3 (a)). It may be that this cluster is statistically significant when viewed as having 10 instances. But in another view, the operons view, this cluster consists of only 6 instances (figure 4.3 (b)). In the operons view, it consists of one operon that has three genes, two operons each having two genes, and three single genes (we can imagine these three genes as three operons, each operon having one gene). With the operons view, the same cluster now has 6 instances as operons, and may be that this cluster is statistically insignificant when viewed as having 6 instances, and it should be treated by MAXCCLUS as if it was generated by chance and therefore should be ignored and not considered as a significant cluster.

From this example, we expect that, when clustering operons, MAXCCLUS will find fewer significant clusters. In order to explore significance, we run MAXCCLUS to cluster operons in this chapter.

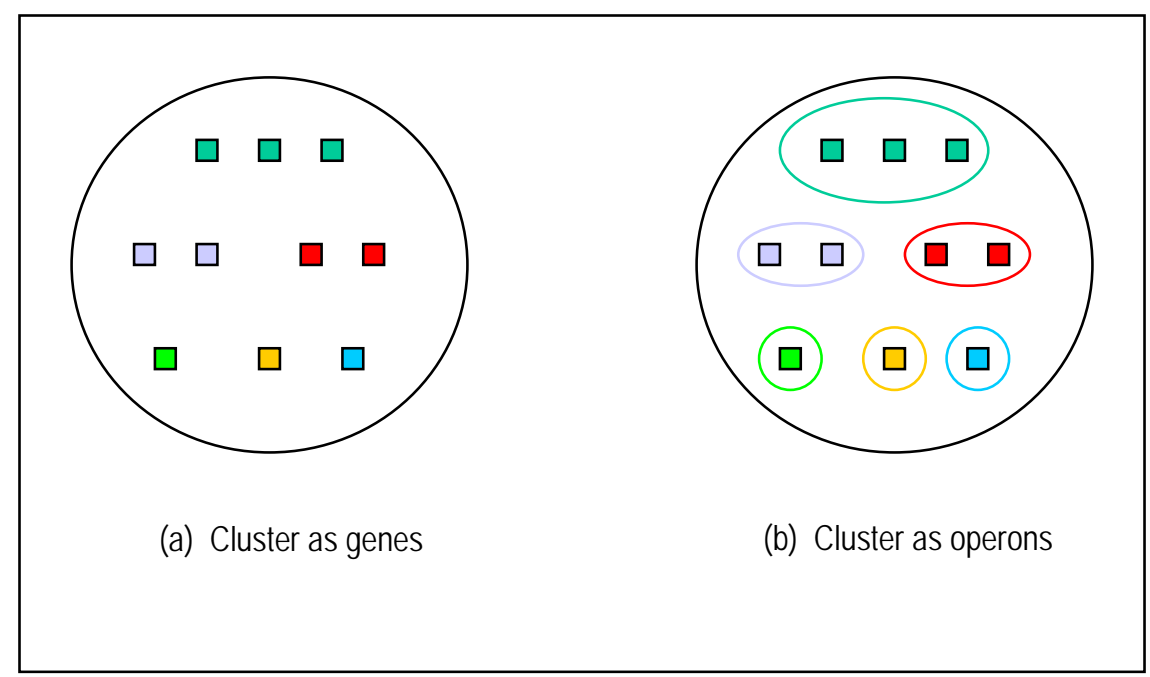

Figure 4.3: Two views for a cluster: (a) as genes, (b) as operons. 


\subsection{Preparing the data}

To be able to run MAXCCLUS on operons instead of genes, we need to prepare the data that MAXCCLUS uses. We have two kinds of data to be used by MAXCCLUS: first the textual data, and second the microarray experimental data.

\subsubsection{Preparing the textual data}

The textual data for operons was prepared by creating the operon descriptor table and storing it to a file to be ready for use by MAXCCLUS. This table contains a number of rows; each row contains an operon's id associated with the operon's descriptors' sets. We created each operon's descriptor set out of its genes descriptor sets. There are two methods to do this. We implemented the second one only.

\section{First method}

The first method is to take the intersection of the descriptor sets of the genes in the operon; this gives us all the words that are true of all the operon's genes. The operon descriptor sets that we get from this method are not rich enough to be useful for our experiment; therefore we do not use it.

\section{Second method}

The second method is to take the union of the descriptor sets of the genes in the operon; this gives us the words that are true of any of the operon's genes. This method reflects the functional dependency between an operon's genes. As we extracted the gene descriptors from a protein database (the SwissProt), the genes descriptors describe the protein that each gene codes for. Because the operon's genes are functionally related, then for each operon, its descriptor set needs to be the union of the descriptor sets of the genes in this operon to keep the knowledge about all the operon's genes. From that, the 
second method creates a richer set of descriptors that describes the operon; therefore, we chose the second method to create the operon descriptor set.

\subsubsection{Preparing the microarray experimental data}

The operon microarray experimental data is a file that contains a number of rows, in which each row contains an operon's id associated with the operon's category. For each microarray experiment, the operon microarray experimental data is created from the gene microarray experimental data for this microarray experiment. The problem is that not all genes of every operon have the same category; some genes are not labelled "Up" or "Down" and it is possible that two genes in the same operon could have opposite categories. Therefore, it is not clear how to assign categories to operons. We took three approaches to creating and implementing the operon microarray experimental data.

\section{First approach (OP100)}

The first approach is to accept an operon (include its id in the operon microarray experimental data) if $100 \%$ of its genes are present in the gene microarray experimental data and if $100 \%$ of its genes are associated with the same category. We then associate this operon's id with the same category as its genes' category. If any genes are not present or their categories are mixed, we reject the operon so it will not be included in the operon microarray experimental data. We will refer to this approach as OP100. We will also use this reference to refer to the results of the operon clustering experiment, which uses operon microarray experimental data created by using this approach.

Using this approach, we only accepted $58 \%$ of the operons that have genes in the gene microarray experimental data. Because this was too strict, we introduced the second approach with fewer constraints. 


\section{Second approach (OP66)}

The second approach is to accept an operon if 66\% or more of all of its genes' are present in the gene microarray experimental data and if $66 \%$ or more of all of its genes are associated with the same category. Then we associate this operon's id with the majority category of its genes. Otherwise we reject the operon. We will refer to this approach as OP66. We will also use this reference to refer to the results of the operon clustering experiment, which uses operon microarray experimental data created by using this approach.

Using the second approach, we accepted $63 \%$ of the operons that have genes in the gene microarray experimental data. While the second approach is a little bit less strict than the first approach, the second approach is still strict. Therefore, we introduce the third approach that is looser in its constraints than the first and the second approaches.

\section{Third approach (OP70)}

The third approach is to accept an operon if any of its genes are present in the gene microarray experimental data as long as $70 \%$ or more of the genes that are present are associated with the same category. Then we associate this operon's id with this category. Otherwise, we reject the operon. We will refer to this approach as $\mathbf{O P 7 0}$. We will also use this reference to refer to the results of the operon clustering experiment, which uses operon microarray experimental data created by using this approach.

Using the third approach we accepted $98.4 \%$ of the operons that have genes in the gene microarray experimental data. This third approach is the least strict of the three approaches regarding constrains for accepting operons in the operon's microarray experimental data. 


\subsection{Experimental results}

The results shown in the figures below are for running MAXCCLUS on 20 sets of the operon microarray experimental data (data set: $6,7,10,11,12,16,18$, $20,21,22,23,24,25,27,28,29,30,35,36$, and 40), and using the operon textual data.

Figures 4.12 to 4.19 show the results for the number of rules, the number of words in the rules, and the number of words per rule. The figures compare the results on the operon data with the corresponding results on gene data. The comparison between the operons data and the genes data is direct.

Figures 4.4 to 4.8 show the results for the number of clusters and the number of instances. However, for these figures the comparison between operons and genes cannot be direct, because the instance ids of genes are different from those of operons. So to be able to compare, we substituted each operon's id by the ids of its genes, and substituted the clusters of operons by the clusters that contain the genes that these operons map to. By doing this, we can now make a valid comparison between the clusters and instances from the operon data and the clusters and instances from the gene data. 


\section{The clusters}

Figures 4.4 and 4.5 show the changes to the number of clusters when the genes are replaced by operons. The figures show the changes to the total number of clusters and the number of clusters subsumed by new clusters.

When using any of the approaches OP100, OP66 or OP70, on average, there are fewer clusters when clustering operons than when clustering genes, specially the significant clusters (figure 4.4).

For any of the cluster sets (significant, cover, or generalised cover cluster set), on average, there are fewer clusters when using OP100 than when using OP66, which in turn, are fewer clusters than when using OP70.

When using any of the approaches OP100, OP66 or OP70, on average, the significant cluster set has the highest decrease in the number of clusters.

When clustering operons, on average, almost no clusters are retained from the clusters found when clustering genes and almost all the clusters are different from the clusters found when clustering genes. On average, very few or no clusters found when clustering genes are subsumed by new clusters found when clustering operons, except for OP70 where there is a noticeable percentage of the number of clusters found when clustering genes subsumed by the new clusters found when clustering operons, especially the significant clusters set (figure 4.5). 


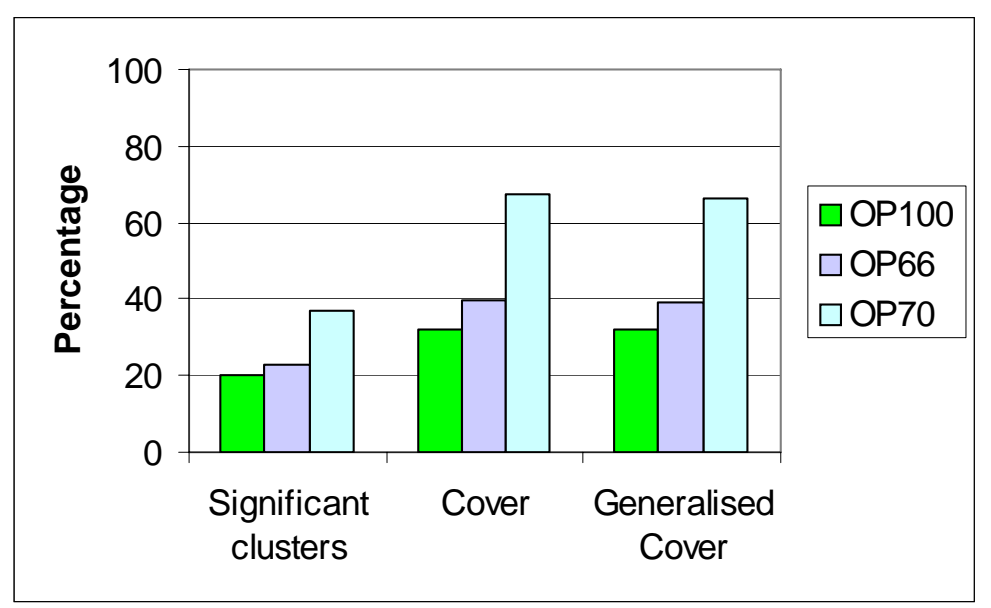

Figure 4.4: Average change to the total number of clusters

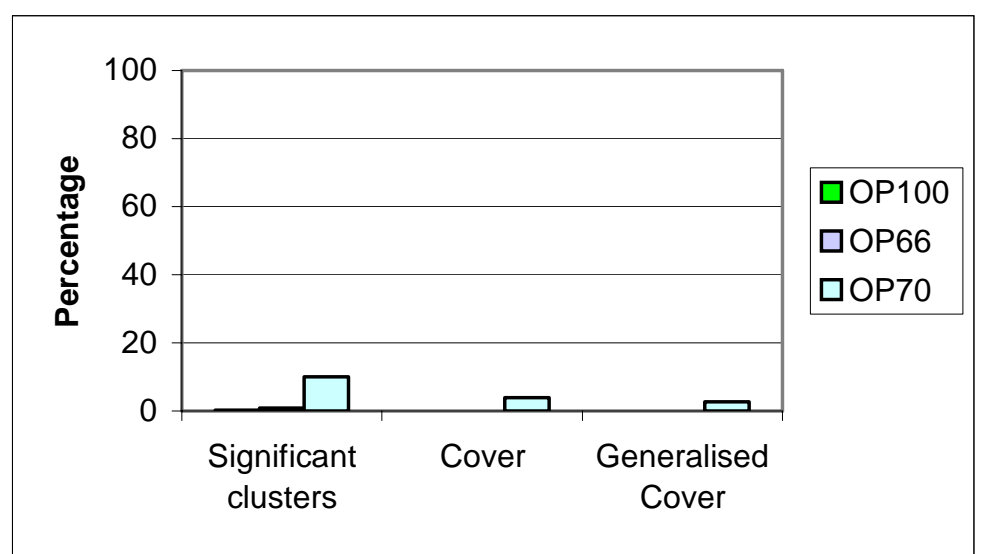

Figure 4.5: Average number of clusters subsumed by new clusters 


\section{The instances}

Figures 4.6 to 4.8 show the changes to the number of instances when the genes are replaced by operons. The figures show the changes to the total number of instances, the number of retained instances, and the number of new instances.

When using any of the approaches OP100 or OP66, on average, there are fewer instances when clustering operons than when clustering genes, specially the significant clusters (figure 4.6). In contrast, on average, when using approach OP70, there are more instances when clustering operons than when clustering genes.

For any of the cluster sets, significant, cover, or generalised cover cluster set, on average, there are fewer instances when using OP100 than when using OP66, which in turn, there are fewer instances than when using OP70.

When clustering operons, on average, about $40 \%$ to $60 \%$ of the instances are retained from the instances found when clustering genes (figure 4.7). For all cluster sets, significant, cover, or generalised cover cluster set, on average, the retained instances when using OP100 are fewer than that when using OP66, which in turn, are fewer than that when using OP70.

For all the cluster sets, significant, cover, and generalised cover cluster set, on average, the new instances that are described when clustering operons, which were not described when clustering genes, are many more when using OP70 than when using OP66, which in turn, are a little bit more than when using OP100 (figure 4.8). 


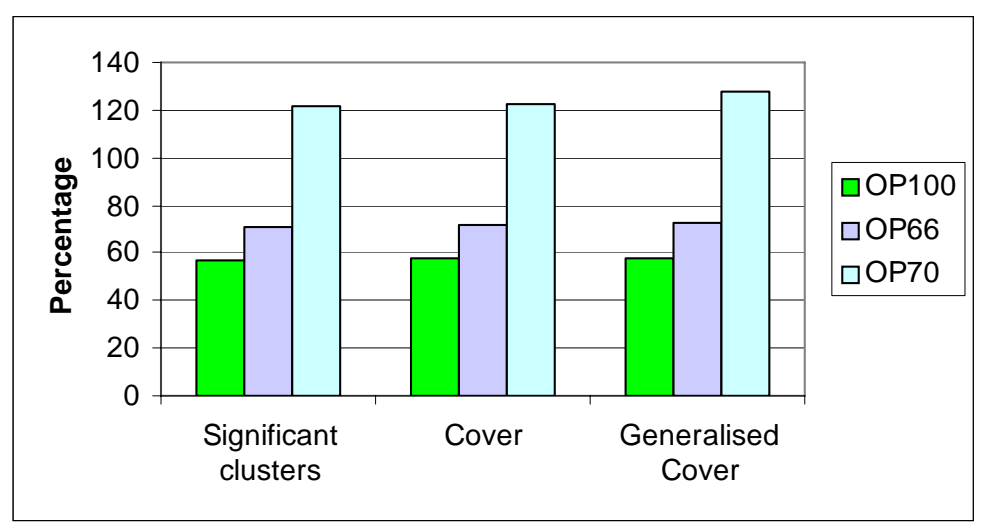

Figure 4.6: Average change to the total number of instances

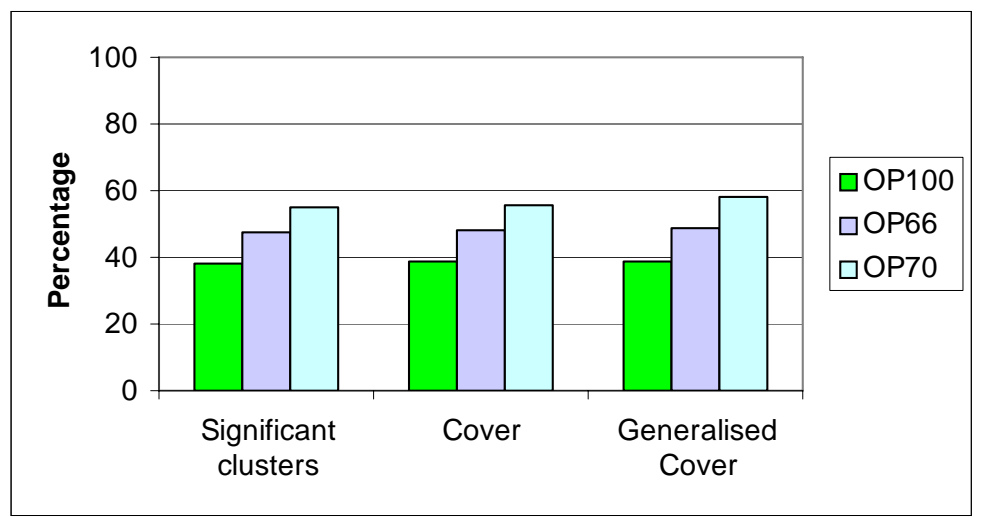

Figure 4.7: Average number of retained instances

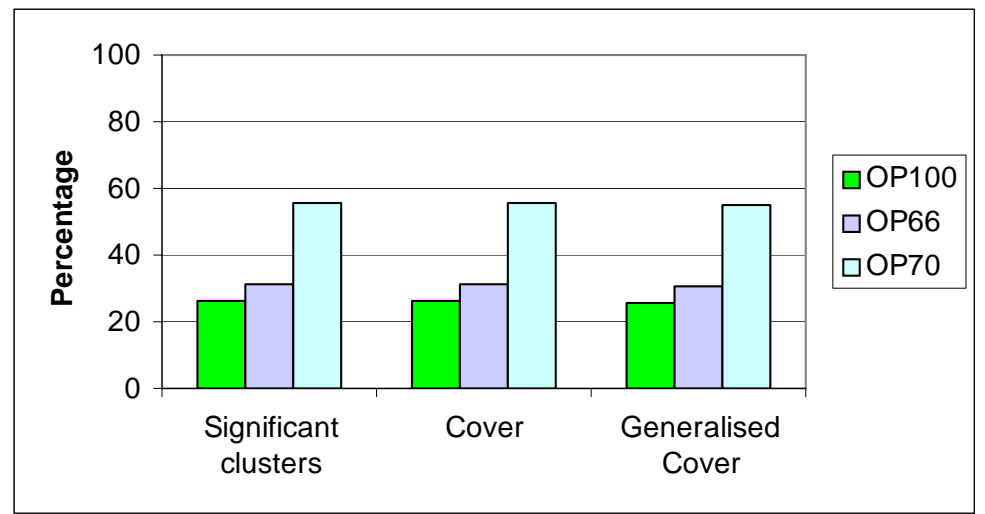

Figure 4.8: Average number of new instances 
Figure 4.9 shows the average percentage of the predicted instances (genes) when clustering operons. The predicted genes are the genes that exist in the gene set mapped from the operons after clustering operons but do not exist in the gene experimental data sets. The percentage of the predicted genes $P \%$ for each data set is calculated as the percentage ratio of the number of predicted genes to the number of the genes from the operons clustering:

$P \%=\frac{|G p|}{|G o|} \times 100$

Where $G p$ represents the predicted genes, and Go represents the genes from operon clustering.

There are no genes predicted when using OP100. On average, the predicted genes when using OP66 are fewer than when using OP70 (figure 4.9).

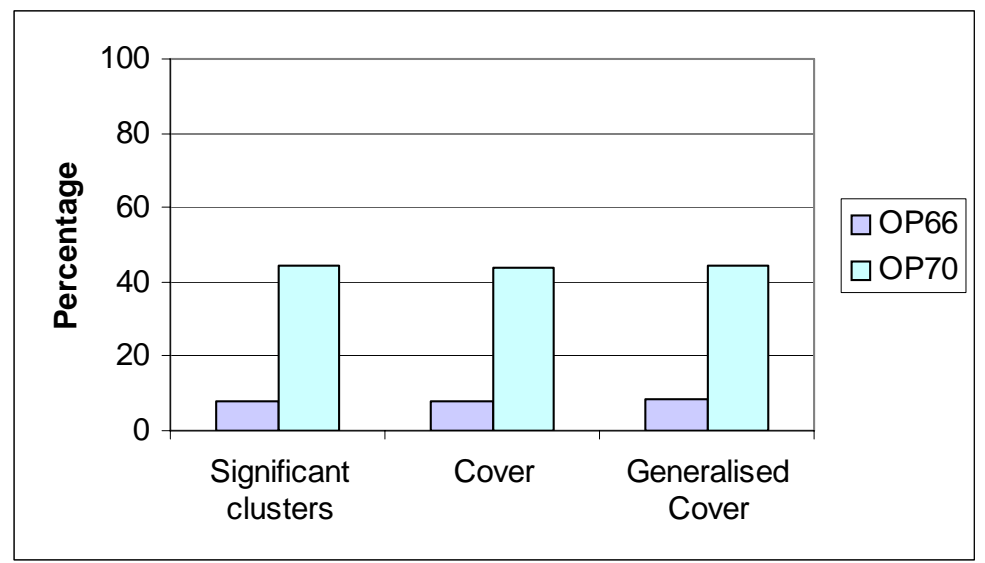

Figure 4.9: Average number of predicted instances 
Figure 4.10 shows the average of the correctly predicted genes when clustering operons. The correctly predicted genes are the predicted genes whose real categories are near their predicted categories. A correctly predicted Up gene is a gene that its expression ratio is between 1.4 and 2.0 (excluding) and was predicted to be Up, while a correctly predicted Down gene is a gene that its expression ratio is between 0.5 and 0.7 (excluding) and was predicted to be Down. The percentage of the correctly predicted genes $C \%$ for each data set is calculated as the percentage ratio of the number of correctly predicted genes to the number of the predicted genes:

$C \%=\frac{\left|C_{G p}\right|}{|G p|} \times 100$

Where $C_{G p}$ represents the correctly predicted genes, and again, $G p$ represents the predicted genes.

There are no correctly predicted genes when using OP100 because there are no predicted genes. On average, the correctly predicted genes when using OP66 are more than that when using OP70 (figure 4.10).

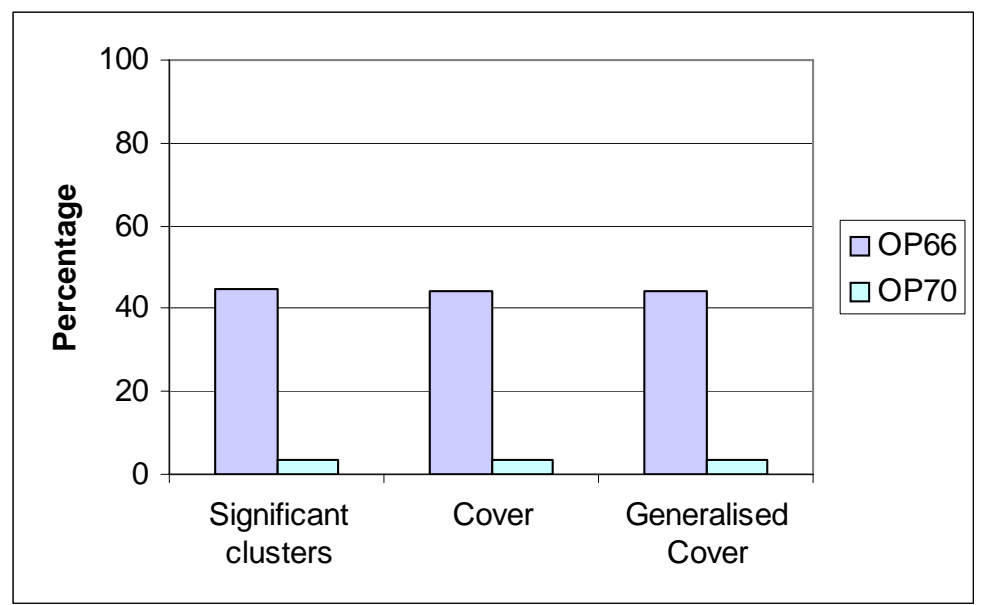

Figure 4.10: Average number of the correctly predicted instances 


\section{The Instances with respect to the experimental data}

Figures 4.6 to 4.8 show the results for all the genes in the clustered operons. But since this includes genes that were not clearly Up or Down in the experimental data sets, the comparisons are not completely fair. An alternative is to confine the comparisons to just the Up and Down Genes in the data sets.

Figure 4.11 shows the number of $U p$ and Down genes described by the rules when clustering operons.

The percentage of the Up and Down described genes $D_{u d} \%$ for each data set is calculated as the percentage ratio of the number of described genes that have categories Up or Down when clustering operons:

$D_{\text {ud }} \%=\frac{\left|G_{\text {Oud }}\right|}{\left|G_{\exp }\right|} \times 100$

Where Goud represents the genes from operon clustering whose categories are Up or Down, and $G$ exp represents the genes from gene experimental data. Figure 4.11 shows these results for the three clustering approaches (OP100, OP66, and OP70). It can be compared with figure 4.6.

When clustering operons, for any cluster set, significant, cover, generalised cover cluster set, the Up or Down described instances are fewer when using OP100 than when using OP66, which in turn, is fewer than when using OP70. The distributions show that there is a relatively wide variation across the data sets especially for OP70. 


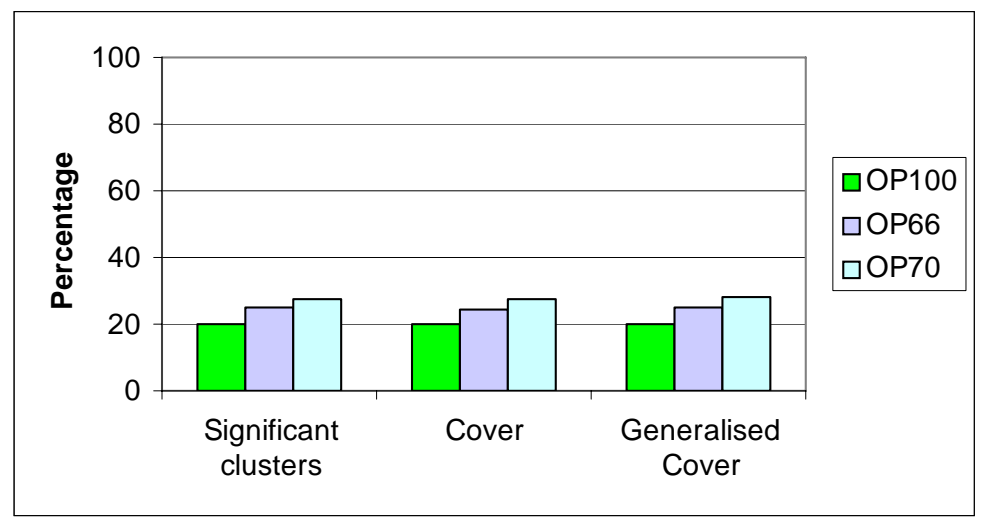

a: Average

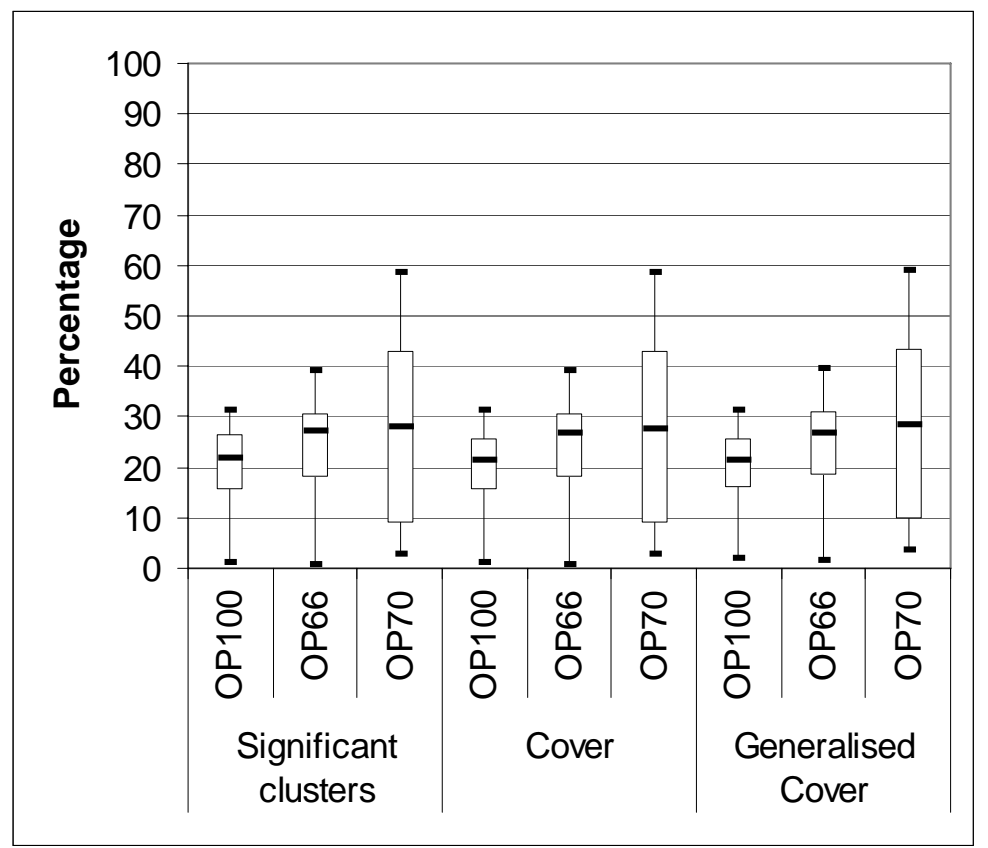

b: Distributions

Figure 4.11 : Number of Up and Down instances described. 


\section{The rules}

Figures 4.12 to 4.15 show the changes to the number of rules when the genes are replaced by operons. The figures show the changes to the total number of rules, the number of retained rules, the number of rules subsumed by new rules, and the number of new rules.

Using any of the approaches OP100, OP66 and OP70, on average, there are fewer rules when clustering operons than when clustering genes (Figure 4.12). There are fewer rules when using OP100 than when using OP66, which in turn, are fewer than when using of OP70.

Using any of the approaches OP100, OP66 and OP70, on average, the significant cluster set has the highest decrease in the number of rules. When using OP70, on average, the cover cluster set has the lowest decrease in the number of rules.

When clustering operons, on average, only a few rules are retained from the clusters found when clustering genes (Figure 4.13). On average, OP70 retained fewer rules than OP100, which in turn, retained fewer rules than OP66.

On average, there are a reasonable number of rules found when clustering genes that are subsumed by new rules found by clustering operons (Figure 4.14). On average, OP70 has fewer rules subsumed than OP66, which in turn, has fewer rules subsumed than OP100. The distributions show that there is a relatively wide variation across the data sets for OP100 significant clusters.

MAXCCLUS replaced the lost rules by new ones (Figure 4.15). MAXCCLUS found a lot of new rules when clustering operons. On average, OP66 has fewer new rules than OP100, which in turn, has fewer new rules than OP70. 
Chapter 4: Operons

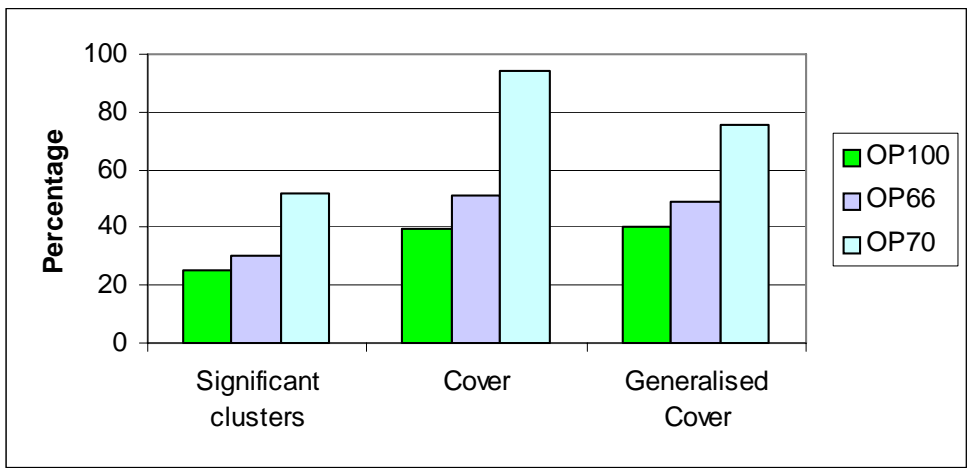

Figure 4.12: Average change to the total number of rules

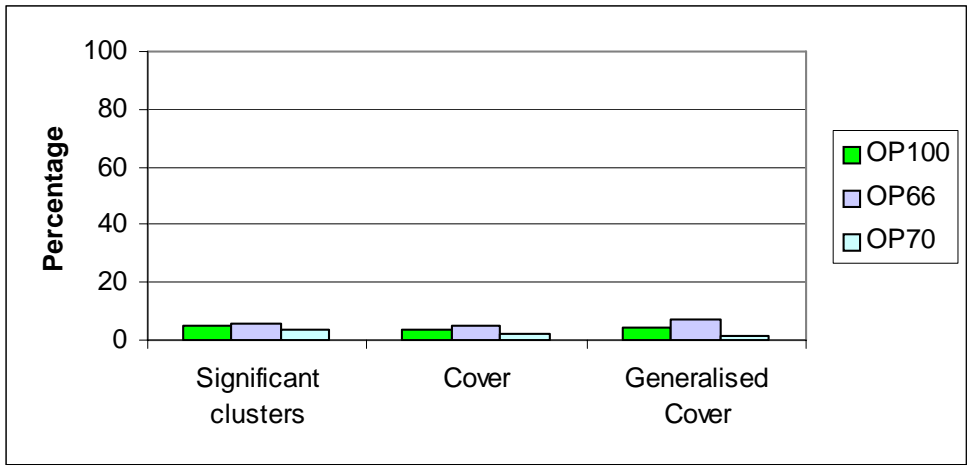

Figure 4.13: Average number of retained rules 


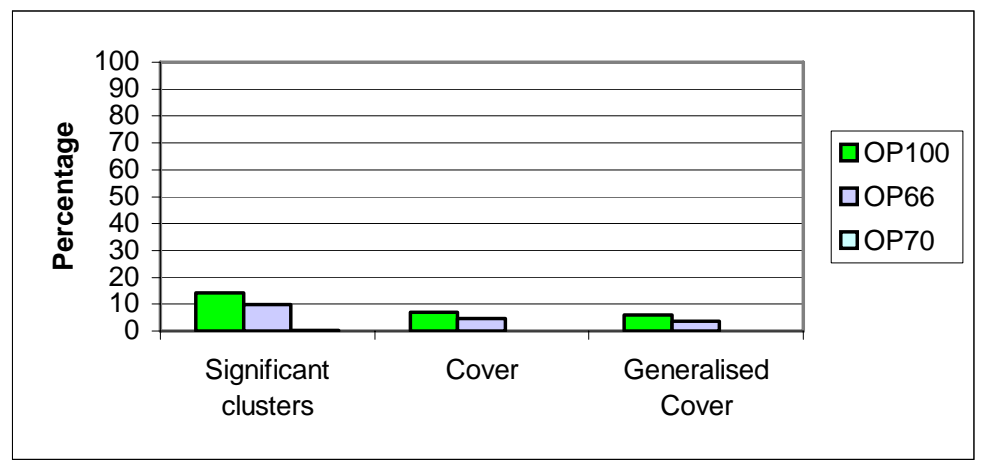

a: Average

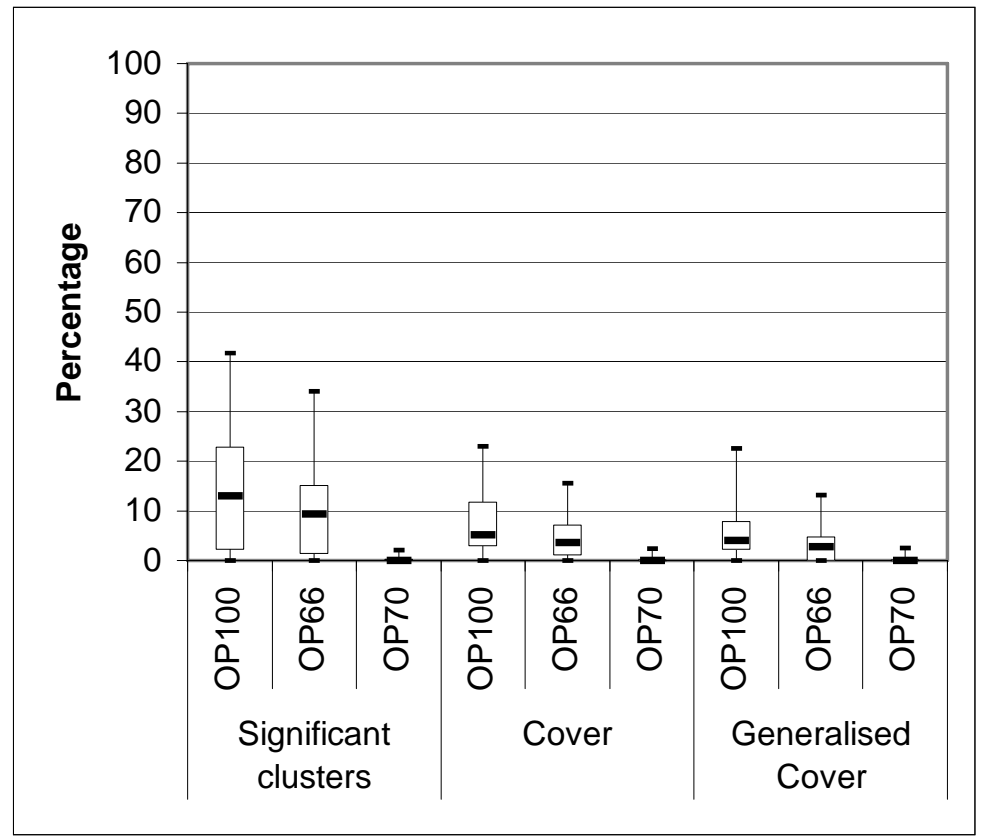

\section{b: Distributions}

Figure 4.14: Number of rules subsumed by new rules

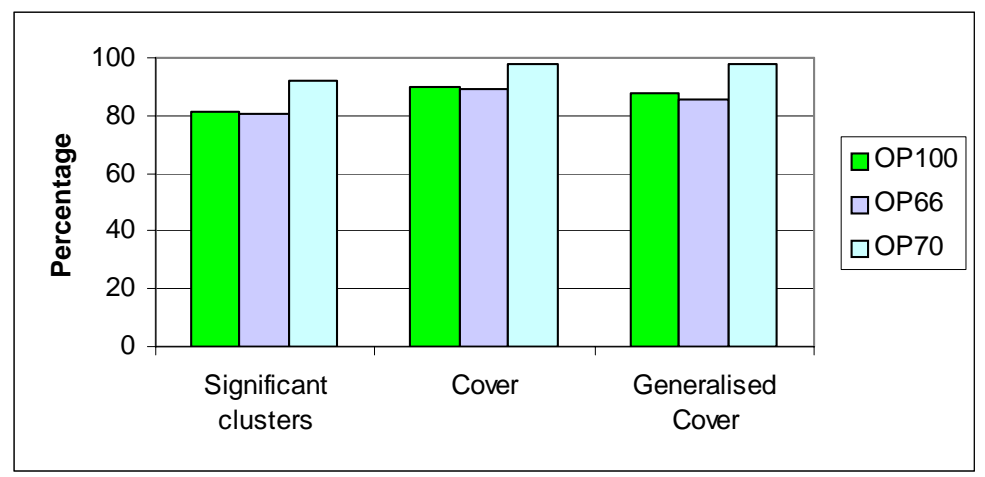

Figure 4.15: Average number of new rules 


\section{The rules' words}

Figures 4.16 to 4.18 show the changes to the number of words in the rules when the genes are replaced by operons. The figures show the changes to the total number, the number of retained words of the rules, and the number of new words.

On average, there are fewer words for rules when clustering operons than when clustering genes (figure 4.16).

On average, OP100 has higher decrease in the number of rules' words than OP66, which in turn, has higher decrease in the number of rules' words than OP70.

When using any of the approaches, OP60, OP66, or OP70, on average, the significant cluster set has the highest decrease in the number of rules' words.

On average, OP100 retained fewer rules' words than OP66, which in turn, returned fewer rules' words than OP70 (figure 4.17).

MAXCCLUS replaced the lost words with new words (figure 4.18).

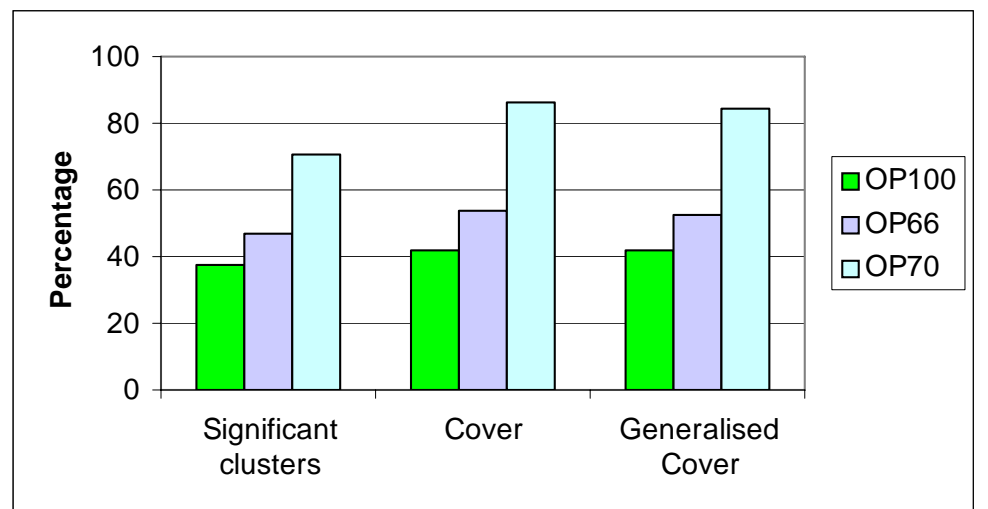

Figure 4.16: Average change to the total number of rules' words 
Chapter 4: Operons

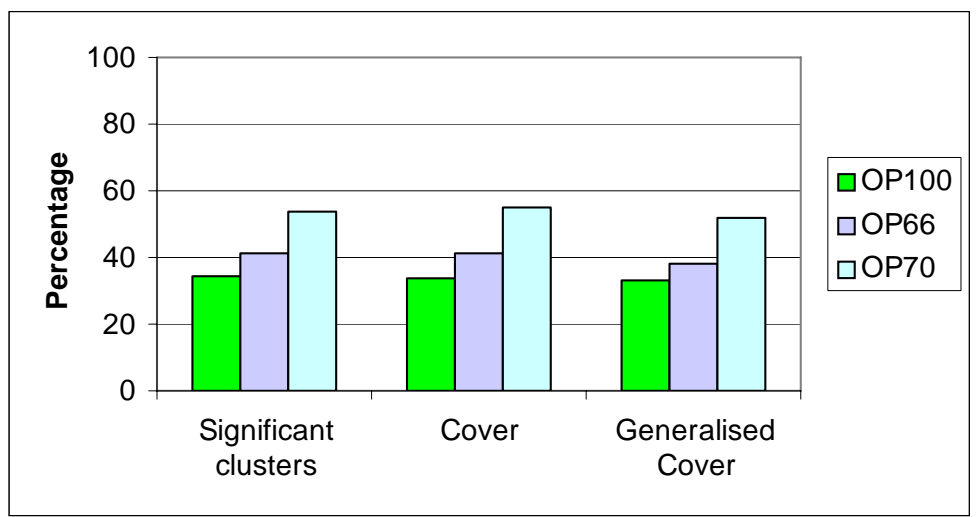

Figure 4.17: Average number of retained rules' words

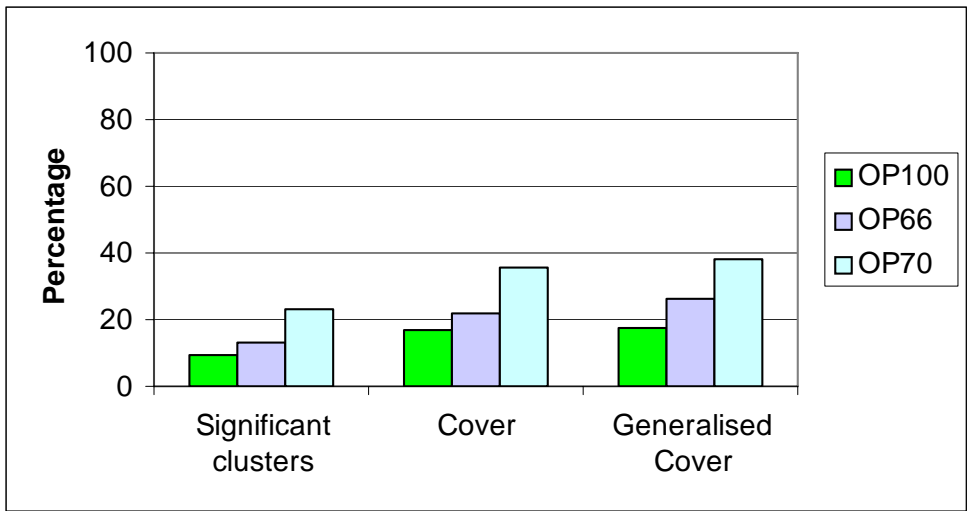

Figure 4.18: Average number of new rules' words 


\section{The words per rule}

Figure 4.19 shows the changes to the number of words per rule when the genes are replaced by operons.

On average, when using the strictest approach OP100, the number of words per rule when clustering operons is less than that when clustering genes. When using the less strict approach OP66, on average, the number of words per rule when clustering operons is almost the same as that when clustering genes. When using weakest approach OP70, on average, the number of words per rule when clustering operons is more than that when clustering genes.

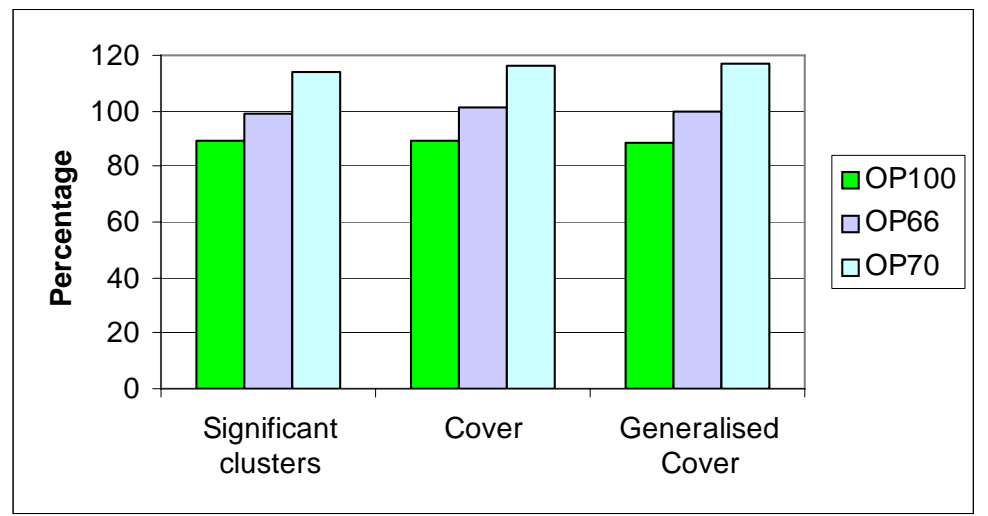

Figure 4.19: Average change to the number of words per rule 


\subsection{Analysis and discussion}

The following sections analyse and discuss the results shown in the results section.

\section{Clusters}

Figure 4.4 shows that the average number of the clusters is smaller when clustering operons than it is when clustering genes, specially the significant clusters.

As we mentioned at the beginning of this chapter and showed in the example of section 4.1, it is not the case when clustering genes that MAXCCLUS finds lots of clusters that correspond directly to operons because there are not many big operons (figure 4.2). However it is the case that some clusters have several genes belonging to the same operon, as the example illustrates. Therefore we have fewer clusters when clustering operons than when clustering genes because MAXCCLUS finds fewer significant clusters when clustering operons than when clustering genes. Notice that when clustering operons, especially when using the OP70 approach, MAXCCLUS did not retain any of the gene clusters, there were very few gene clusters subsumed by operon clusters, and almost all the operon clusters were new. Dropping all the clusters and replacing them with new ones could be because of using the union of the descriptor sets of the genes when creating the descriptor sets of the operons. Or it could be because accepting operons that contain genes whose categories are not clearly Up or clearly Down, will produce a new set of clusters. Some of these new clusters subsumed old clusters of the gene clustering (figure 4.5). 


\section{Instances}

For the instances, when clustering operons using OP100 or OP66, on average, MAXCCLUS is able to describe fewer genes than it can when clustering genes (figure 4.6). In contrast when using OP70, on average, MAXCCLUS is able to describe more genes than it can when clustering genes. Notice that with OP100, which is the strictest approach, MAXCCLUS describes fewer than the other approaches. This is because in OP100 we included in the operon microarray experimental data only the operons that are $100 \%$ pure, therefore we had fewer instances to cluster; in OP66 we included operons that are less than $100 \%$ pure, so there were more genes to be described.

An example is that we can have an operon of 10 genes of which nine genes have one category and the other one gene has a different category. By using the OP100 approach this operon (and the other similar operons) would be rejected. But by using the OP66 approach this operon would be accepted. Having a different category for the single gene could be a result of noise or wrong measurements while carrying out the microarray experiment. Therefore it is possible this gene would have the same category as the other genes in the operon if there was no noise or the measurements were right.

Including these extra operons, when using the OP66 approach, added extra genes that were not categorised as clearly Up or clearly Down genes (and so were not included in the gene microarray experimental data) although they might have been nearly Up or nearly Down. Including these extra operons helps to correct the noise or the wrong measurements in the microarray experimental data, and to predict that those genes whose categories were not clearly Up regulated should have been Up regulated, and predict that the ones that were not clearly Down regulated should have been Down regulated.

When using OP66, on average, about 8\% of the genes that MAXCCLUS can describe are predicted genes (figure 4.9). About $45 \%$ of these predicted 
genes are correctly predicted (figure 4.10). Correctly predicted genes are the genes whose real categories are near Up or near Down, when MAXCCLUS predicted them to be Up or Down respectively.

By being even less strict in selecting operons in the operon microarray experimental data, as in OP70, MAXCCLUS is able to describe more genes than it can describe using the stricter two approaches. Because with OP70 more operons are accepted than with OP66, the new included operons may have many more genes with categories that are neither Up nor Down. These categories can be in-between the Up and Down categories but not so close to Up or to Down. For each operon, MAXCCLUS predicts that the operon's genes that are not in the gene experimental data are regulated Up (or Down) on the basis that the operon has $70 \%$ or more of its genes that are clearly regulated Up (or Down). To feel how less strict is the OP70 approach we can have an example.

In this example, we have an operon that has 15 genes. Suppose only one of these genes is in the gene experimental data and this gene has the Up category. Then when creating the operon experimental data with the OP70 approach, this operon will be accepted and it will be assigned the Up category even though it has 14 other genes with unknown categories of which some may not be even near Up, and may even be near Down. In contrast, OP66 would reject this operon because only about $7 \%$ of its genes are Up regulated.

When MAXCCLUS predicts that the 14 other genes of the operon in the above example have Up categories, even though some of them are far away from being Up, this prediction is not at all reliable. It is probable that some of the 14 genes have categories that are near Up, but it is much less probable for all of them to have categories that are near Up. 
When using the OP70 approach in accepting operons in the operon microarray experimental data, on average, it seems that there is an increase in the number of genes that MAXCCLUS can describe when clustering operons than when clustering genes (figure 4.6). But this is not the case when we look only at the genes of the gene microarray experimental data (figure 4.11 OP70). In this result, when clustering operons MAXCCLUS covers fewer genes than when clustering genes (figure 2.4). In figure 4.6, the extra genes of OP70 described by MAXCCLUS are predicted genes (figure 4.9). Although, when using OP70 MAXCCLUS is able to predict many more genes than when using OP66 (figure 4.9), on average, the correctly predicted genes when using OP70 are much fewer than when using OP66 (figure 4.10). So, on average, when using OP70 most of the genes that MAXCCLUS predicts to have Up or Down categories are the result of incorrect prediction (on average, only $3 \%$ of the predicted genes are correctly predicted). In contrast, when using OP66 about 45\% of the predicted genes are correctly predicted by MAXCCLUS, which make using the OP66 approach more sensible than using the OP70 approach in accepting operons in the operon microarray experimental data.

We have considered MAXCCLUS's predictions for genes when using OP66 or OP70, but not when using OP100 because for OP100 the operons are accepted in the operon microarray experimental data are 100\% pure and each operon contains genes that are all Up or are all Down. Therefore, there is no prediction issue when using OP100.

In summary, on average, when clustering genes, MAXCCLUS can explain about $37 \%$ of the genes (figure 2.4). But when grouping genes into operons and clustering these operons, on average, MAXCCLUS can explain only about $20 \%$ of the genes using the strictest approach OP100 (figure 4.11). This percentage increases a little by using the less strict approach OP66, and again increases by using the least strict approach OP70. This increase is because of accepting more operons that will introduce more genes; these 
genes were not present in the operons accepted using the strictest version OP100.

\section{Comparing clusters results with instances results}

When clustering with operons the number of clusters was lower than when clustering with genes. At the same time, the number of genes went down but not as much as the number of clusters. That means when grouping genes into operons and then clustering these operons MAXCCLUS finds bigger clusters that have more genes.

\section{Rules}

The number of rules is smaller when clustering operons than when clustering genes. That means when grouping genes into operons and clustering these operons, MAXCCLUS has got rid of some of the very specific rules. These rules had lots of words that probably had not very many instances. These rules were significant when clustering genes, but they are not significant when clustering operons because these rules represent clusters that are too small to be considered significant. Some of these rules represent groups of genes that were mostly operons. Therefore the rules that MAXCCLUS creates when clustering operons are really significant, especially when using the OP100 approach that accepts pure operons, so we get pure rules from this approach. But not all the rules that MAXCCLUS creates when clustering genes are significant because some of them result from groups of genes that belong to operons. 


\section{Comparing instances results with rules results}

For OP100 and OP66, on average, between 57\% and $72 \%$ as many genes are being explained but only between $25 \%$ and $49 \%$ as many rules are explaining those genes (figures 4.6 and 4.12). So there are fewer rules that each explains more genes. This implies that when clustering operons MAXCCLUS creates rules which are bigger and better. MAXCCLUS has retained rules that explain many more genes than when clustering genes. When clustering operons, MAXCCLUS has got rid of the useless rules that explain groups of genes that in reality belong to operons.

\section{Words per rule}

For OP70 (figure 4.19), the results showed that the number of words per rule for the significant cluster set is less than that of the cover cluster set, which in turn is slightly less than that of the generalised cover cluster set. This implies that when creating the cover clusters and generalised cover clusters, MAXCCLUS is creating rules that have more words to describe the instances covered by these clusters. These words come from the descriptors of the extra instances described by the rules.

\subsection{Conclusion}

As described earlier in this chapter, some genes are related to each other. Each group of the related genes is called an operon. The operon's genes are instances that have shared dependency (the operon's genes functionally depend on each other). By clustering genes, MAXCCLUS ignores the dependency between operon's genes during the significance test. This yields clusters that are statistically big enough to be considered as significant clusters. 
When grouping genes into operons, then using MAXCCLUS to cluster the operons, MAXCCLUS could not describe as many genes as when we used it to cluster genes. This drop in the number of genes that MAXCCLUS can describe is because MAXCCLUS is now considering the dependency between some of the genes, which represent operons, during its significance test. But the rules it did obtain were larger (describing more genes) and perhaps more informative. Also, with OP66 and OP70, it is able to make predictions about genes that had ambiguous regulation levels.

\subsection{Recommendations}

MAXCCLUS significance test needs to be modified to consider the dependency of operons' genes, so that a cluster will not be considered significant only because it is statistically big enough, but also because it consists of enough number of independent instances. 


\section{Chapter 5}

\section{Deleting Descriptors}

This chapter describes a clustering experiment designed to study the effects on MAXCCLUS clustering after deleting some of the descriptors of the instances, and comparing its results with the reference clustering experiment where no descriptors are deleted. The deleted descriptors are the non-domain words that we expect to carry little meaning for characterising the clusters. The result of this chapter is to show that deleting the non-domain descriptors can improve MAXCCLUS clustering by allowing MAXCCLUS to create clusters that are no longer characterised by the non-domain words and to generate the clusters faster. Instead of the non-domain words, the domainrelevant words appear in the rules that characterise the clusters. For the reference clustering experiment, these domain-relevant words are frequently excluded from the rules that characterise the clusters because of the presence of the non-domain words in the descriptors of the instances.

\subsection{The experiment}

To carry out this chapters clustering experiment, we first prepared the new textual data (new descriptor table file), then we ran MAXCCLUS on the microarray experimental data sets using the new textual data. 
The normal textual data (the one that has no descriptors deleted from it) that the reference clustering experiment uses is prepared by cleaning the text extracted from the Swiss-Prot database, and deleting the 15 most common function words (stop words): "a", "an", "and", "are", "at", "by", "for", "from", "in", "is", "of", "on", "the", "to", and "with". These function words were removed because they are not informative in the rules that describe the clusters, and because they are so common, they may mask more informative words to be included in the rules.

In this chapter's clustering experiment we choose to clean the textual data further, for the same reason that we deleted the stop words above. We expect that further cleaning of the textual data will improve the scientific relevance of the rules by including more informative words. We also expect cleaning the textual data further will increase the efficiency of the clustering process; because there are fewer descriptors, the clustering execution time will be reduced and the computer memory needed for the clustering process will also be reduced. However, we expected that the number of clusters would not reduce very much so that MAXCCLUS should not lose important clusters.

The project chose two approaches to deleting descriptors from the original descriptor set of each instance:

- Deleting descriptors that exist in a relatively small dictionary. We chose words in the bible for this purpose.

- Deleting descriptors that exist in a relatively large dictionary. We chose WordNet for this purpose.

\subsubsection{The bible-words dictionary}

The bible-words dictionary contains all the words from a "Basic English" version of the bible [Bible in Basic English]. We chose the bible-words dictionary because we expect its words to be far from the domain of biology 
and genetics where we apply our clustering experiments, and because it is relatively small dictionary. We reduced the size of the bible-words dictionary further by excluding all capitalised nouns (the names), for two reasons. First, we expect these names to not occur in the Swiss-Prot data, and second to make the process of deleting descriptors more efficient. The final version of the bible-words dictionary, used in deleting descriptors, contains 3561 words (about 1000 root words).

\subsubsection{WordNet}

WordNet is a lexical database for the English language from Princeton University [Princeton University, 2003]. We chose WordNet as an alternative to the bible-words dictionary because WordNet is much bigger than the biblewords dictionary (WordNet contains 144,309 words). At the same time, we still expect WordNet not to contain many domain-relevant words from genetics. WordNet contains only open class words (nouns, verbs, adjectives and adverbs). Note that because WordNet does not contain function words (such as the word "this"), WordNet is not a strict superset of the bible-words dictionary.

\subsection{The algorithm for deleting descriptors}

To delete the descriptors that exist in the dictionary, we created a program using the Perl computer-programming language.

Figure 5.1 shows the algorithm that attempts to reduce the size of the descriptor set of each instance in the descriptor table file, by going through each descriptor in the instance's descriptor set, and checks if this descriptor exists in dictionary. If the descriptor is in the dictionary, the algorithm deletes the descriptor from the instance's descriptor set. If the resulting descriptor set is not empty, the algorithm saves the instance and the new version of the 
instance's descriptor set to the new descriptor table file, which MAXCCLUS will use in this chapter's clustering experiment. Checking whether a word exists in the bible-words dictionary is straight forward, but because WordNet is not a simple list of words, the program uses a module to interface with the WordNet database [Rennie, J. 2002].

The output data will be the new descriptor table file, in which each line contains an instance id (gene id) followed by a set of descriptors (words that describe the instance). This set of descriptors will lack some descriptors that were in the instance's original descriptor set. A possible consequence is that some instances may be lost from this table if their descriptor sets contained only words in the dictionary. Practically, none of the instances were lost, neither by using the bible-words dictionary nor by using WordNet.

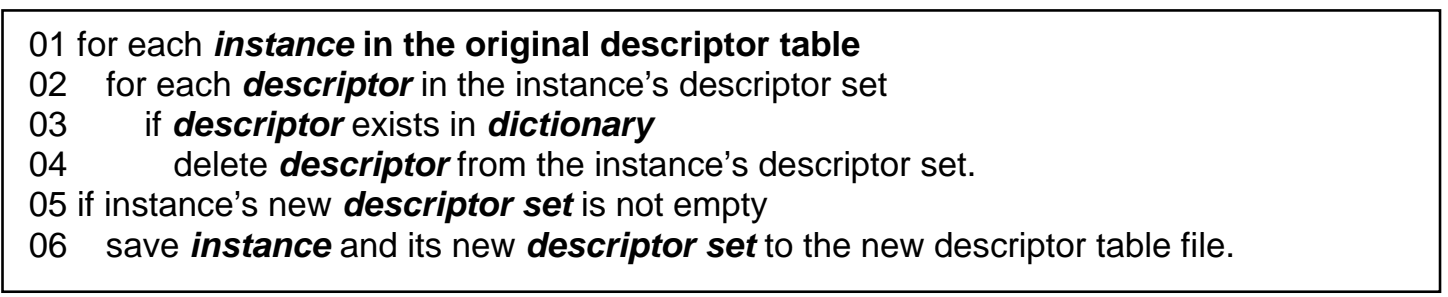

Figure 5.1: The algorithm for deleting descriptors. 


\subsection{Experimental results}

This section and its figures show the results for running MAXCCLUS on 23 of the microarray experimental data sets (data set: $3,6,7,8,10,11,12,13,16$, $18,20,21,22,23,24,25,27,28,29,30,35,36$ and 40) first, after deleting descriptors using the bible-words dictionary, and second, after deleting descriptors using WordNet. We will refer to the two sets of results by $B \boldsymbol{W}$ and $W N$ respectively in the sections and figures below. This section also compares these results with each other and with the results produced by the reference clustering experiment (the one before deleting descriptors). Figures 5.2 to 5.17 show the results for the number of clusters, the number of instances, the number of rules, the number of rules' words, the number of words per rule, and the execution time.

The set of all-descriptors - the union of the descriptor sets of all instances in the descriptor table file - contains 14429 words before deleting descriptors. After deleting descriptors from each instance's descriptor set, the size of the set of all-descriptors reduces also.

There are 394 words from the bible-words dictionary in the set of alldescriptors before reducing the size of the set of all-descriptors. Therefore the set of all-descriptors after deleting from it the descriptors that exist in the bible-words dictionary shrank to approximately $97 \%$ of its original size.

On the other hand there are 2566 words from WordNet in the set of alldescriptors. Therefore the set of all-descriptors after deleting from it the descriptors that exist in WordNet shrank to approximately $82 \%$ of its original size. 


\section{The clusters}

Figures 5.2 to 5.5 show the changes to the number of clusters when some descriptors are deleted from the descriptor sets of the instances. The figures show the changes to the total number of clusters, the number of retained clusters, the number of clusters subsumed by new clusters, and the number of new clusters.

When using BW or WN, on average, there are fewer clusters when clustering after deleting descriptors than when clustering before deleting descriptors (figure 5.2). However, the change in the BW experiment is small: there is almost no change to the number of the generalised cover clusters of BW.

For any of the cluster sets, significant, cover, or generalised cover cluster set, on average, there are more clusters when using BW than when using WN.

When using BW, on average, the significant cluster set has a slightly greater change in the number of clusters, than the cover cluster set, which in turn, has greater change than the generalised cover cluster set. In contrast, when using $\mathrm{WN}$, on average, the significant cluster set has a significantly greater change than the cover and the generalised cover cluster sets.

When clustering after deleting descriptors, on average, the clusters retained from the clusters found when clustering before deleting descriptors, are many more when using BW than when using WN. Between $47 \%$ and $59 \%$ of clusters are retained when using BW, but only between $2 \%$ and $6 \%$ of clusters are retained when using WN. For both BW and WN the generalised cover cluster set retained more clusters than the cover cluster set (figures 5.3).

When clustering after deleting descriptors, on average, the new clusters found are fewer when using BW than when using WN. Between only $39 \%$ and $51 \%$ of clusters are new when using BW, but between $91 \%$ and $95 \%$ of clusters are new when using WN (figure 5.5). 
On average, more clusters found from clustering before deleting descriptors, are subsumed by new clusters found from clustering after deleting descriptors using BW than that using WN. Between $1 \%$ and $19 \%$ of clusters are subsumed when using BW, but only between $0 \%$ and $9 \%$ of clusters are subsumed when using WN. Significant cluster set has noticeable number of subsumed clusters especially when using BW. The significant cluster set has more clusters subsumed than the cover cluster set, which in turn has more clusters subsumed than the generalised cover cluster set, which in turn has almost no clusters subsumed using BW or has no clusters subsumed using WN (figure 5.4).

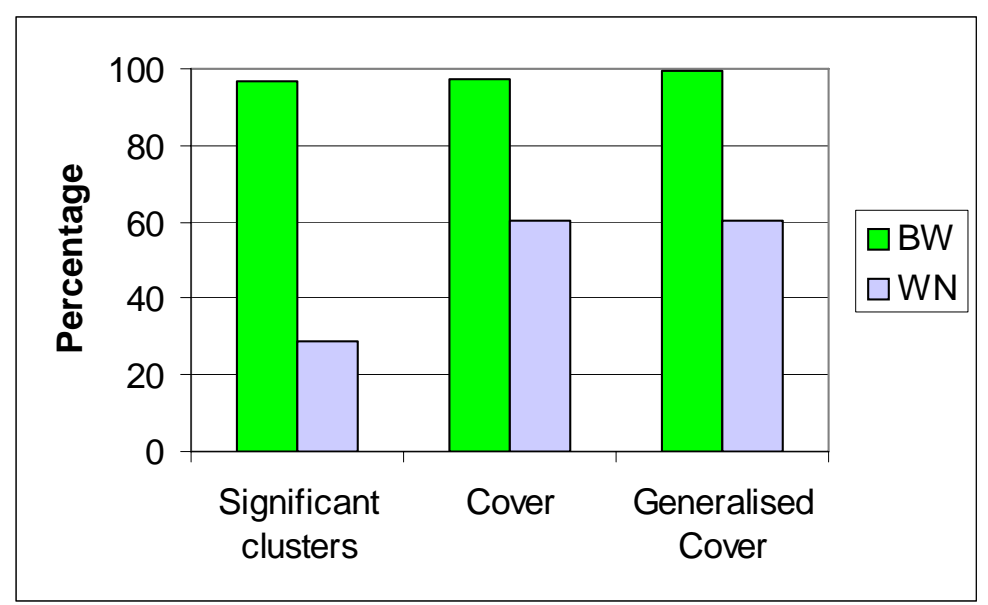

Figure 5.2: Average change to the total number of clusters 


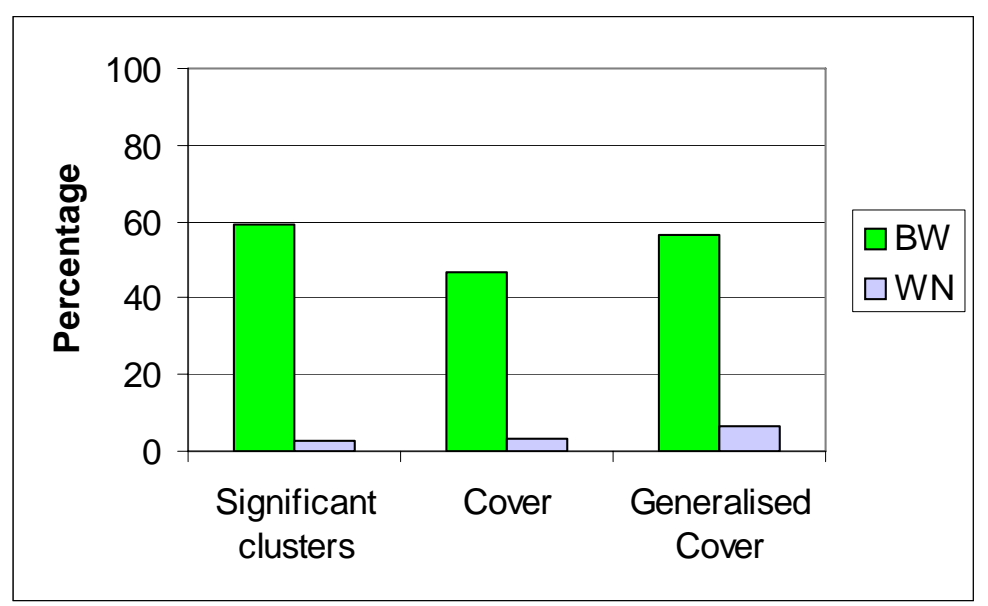

Figure 5.3: Average number of retained clusters

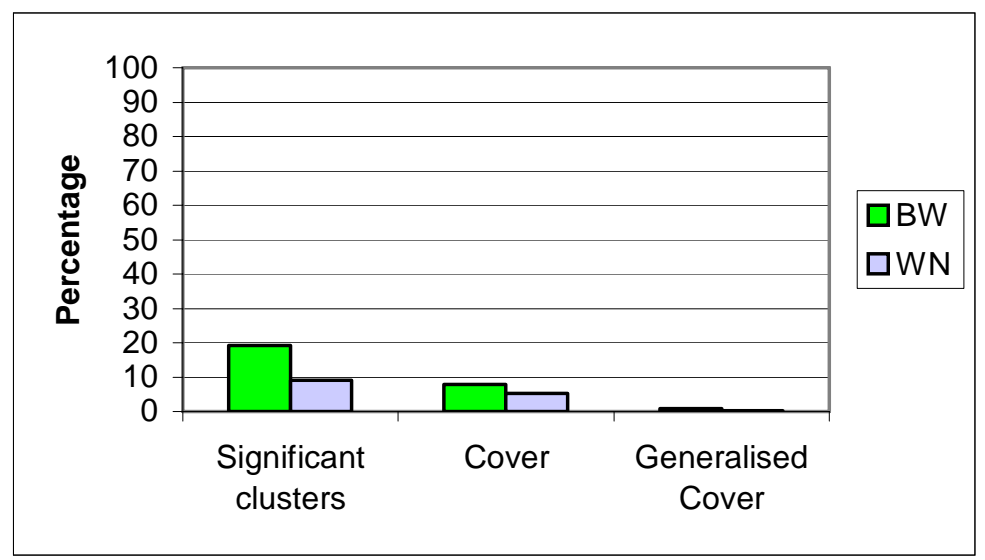

Figure 5.4: Average number of clusters subsumed by new clusters

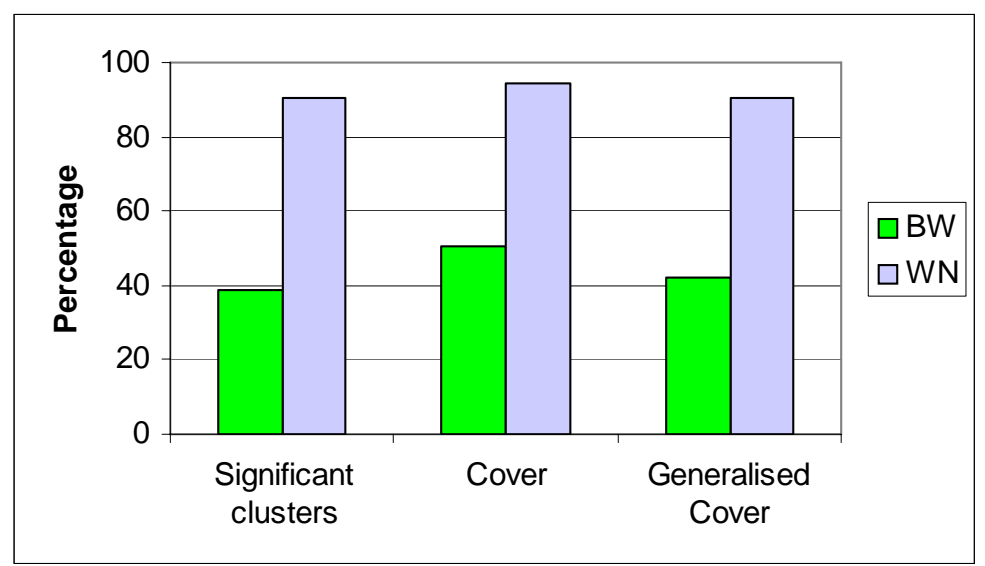

Figure 5.5: Average number of new clusters 


\section{Significant clusters minimum size}

Figure 5.6 shows the changes to the significant clusters' minimum size for each category when some descriptors are deleted from the descriptor sets of the instances.

When clustering after deleting descriptors using BW or WN, on average, the significant clusters' minimum size for each category is less than that when clustering before deleting descriptors. On average, the reduction in the significant clusters' minimum size, when clustering using BW, is less than that when using WN; with almost no change in the minimum size for Down category when using BW. When using any of BW or WN, the reduction in the significant clusters' minimum size for the Up category is more than that for the Down category.

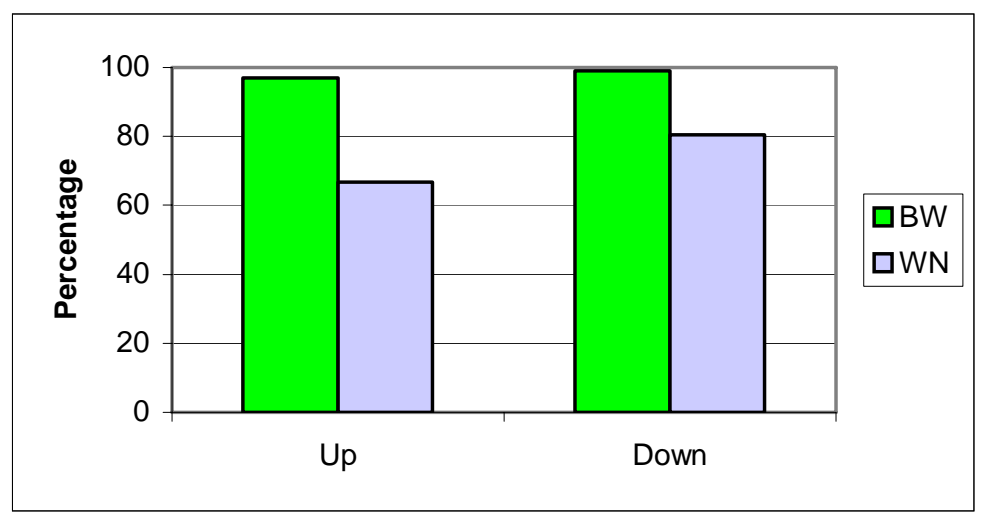

Figure 5.6: Average change to the significant clusters' minimum size for each category 


\section{The instances}

Figures 5.7 to 5.9 show the changes to the number of instances when some descriptors are deleted from the descriptor sets of the instances. The figures show the changes to the total number of instances, the number of retained instances, and the number of new instances.

On average, when using BW, there is almost no change to the number of instances when clustering after deleting descriptors than when clustering before deleting descriptors. In contrast, when using WN, there are fewer instances when clustering after deleting descriptors than when clustering before deleting descriptors, with between only $55 \%$ and $70 \%$ as many instances found when clustering after deleting descriptors than when clustering before deleting descriptors (figure 5.7). When using $\mathrm{WN}$, on average, the generalised cover cluster set has the highest decrease in the number of instances.

When clustering after deleting descriptors, on average, the instances retained from the clusters found when clustering before deleting descriptors, are more when using BW than when using $\mathrm{WN}$, about $94 \%$ of instances are retained when using BW, but only between $45 \%$ and $60 \%$ of instances are retained when using $W N$. For $W N$ the generalised cover cluster set retained fewer instances than the other cluster sets (figures 5.8).

When clustering after deleting descriptors, on average, very few instances found are new. The new instances found are fewer when using BW than when using WN, with about only 5\% of instances are new when using BW, but about $12 \%$ of instances are new when using WN (figure 5.9). 


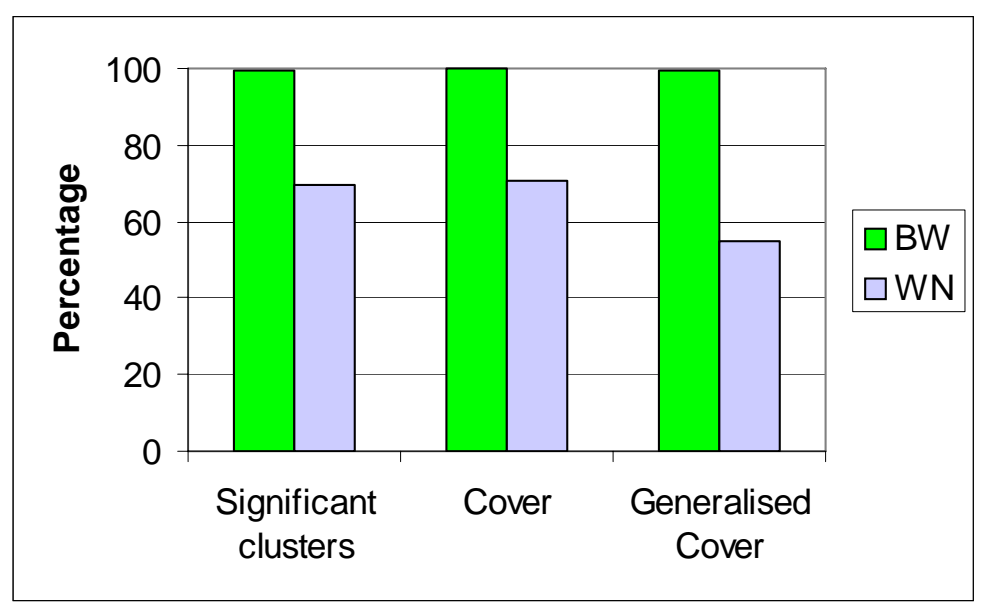

Figure 5.7: Average change to the total number of instances

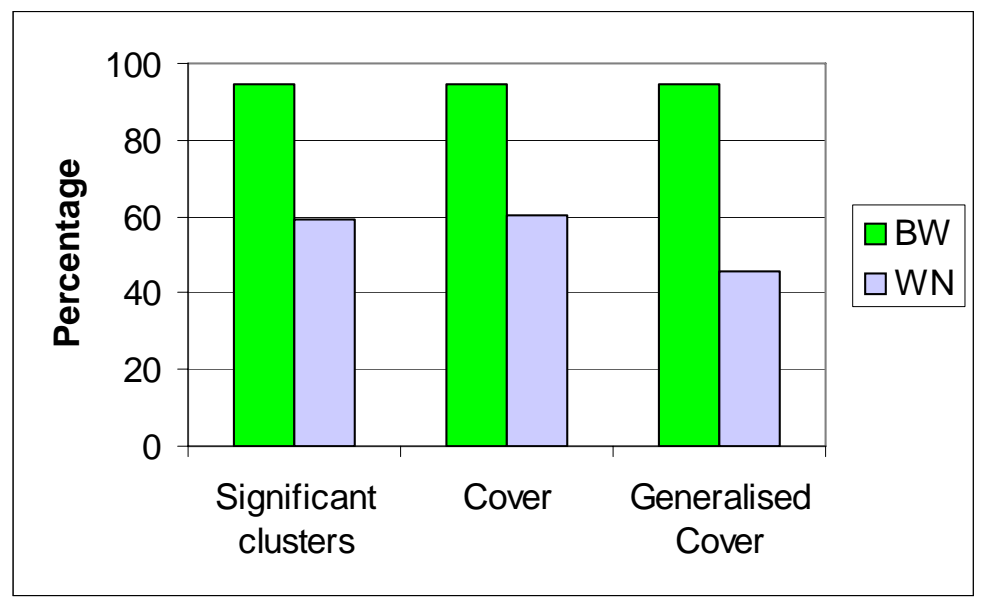

Figure 5.8: Average number of retained instances

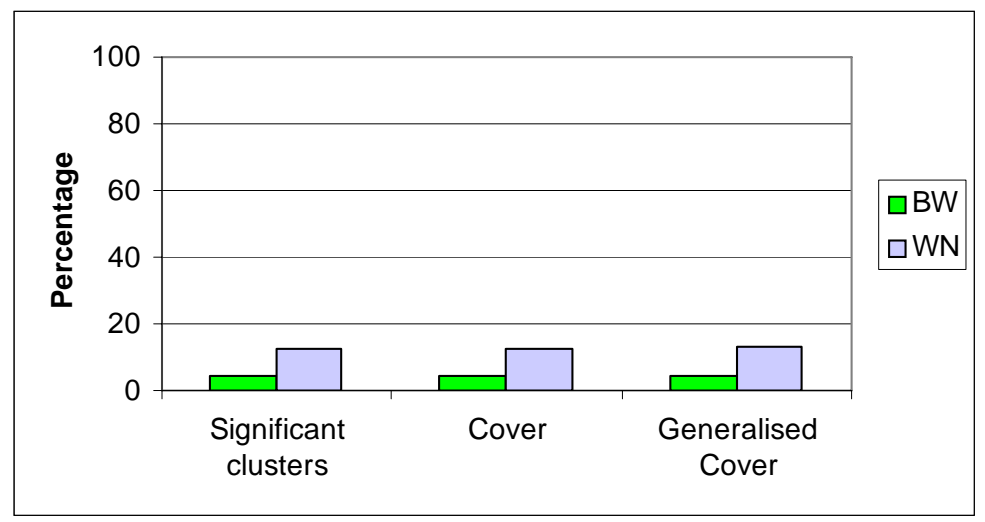

Figure 5.9: Average number of new instances 


\section{The rules}

Figures 5.10 to 5.12 show the changes to the number of rules when some descriptors are deleted from the descriptor sets of the instances. The figures show the changes to the total number of rules, the number of retained rules, and the number of new rules.

When using BW or WN, on average, there are fewer rules when clustering after deleting descriptors than when clustering before deleting descriptors (figure 5.10). On average, there are more rules when using BW than when using WN, between $67 \%$ and $90 \%$ as many rules when using BW, but only between $17 \%$ and $53 \%$ as many rules when using $\mathrm{WN}$.

When using BW or WN, on average, the significant cluster set has greater decrease, in the number of rules, than the cover cluster set, which in turn, has greater decrease than the generalised cover cluster set.

When clustering after deleting descriptors, on average, the rules retained from the clusters found when clustering before deleting descriptors, are many more when using BW than when using WN. Between $38 \%$ and $46 \%$ of rules are retained when using BW, but only about $4 \%$ of rules are retained when using WN. For BW, the significant cluster set retained more rules than the generalised cover cluster set, which in turn, retained more rules than the cover cluster set (figures 5.11).

When clustering after deleting descriptors, on average, the new rules found are fewer when using BW than when using WN. Between only $30 \%$ and $47 \%$ of rules are new when using BW, but between $81 \%$ and $91 \%$ of rules are new when using WN (figure 5.12).

Almost no rules found from clustering before deleting descriptors, are subsumed by new rules found from clustering after deleting descriptors using BW or WN. 


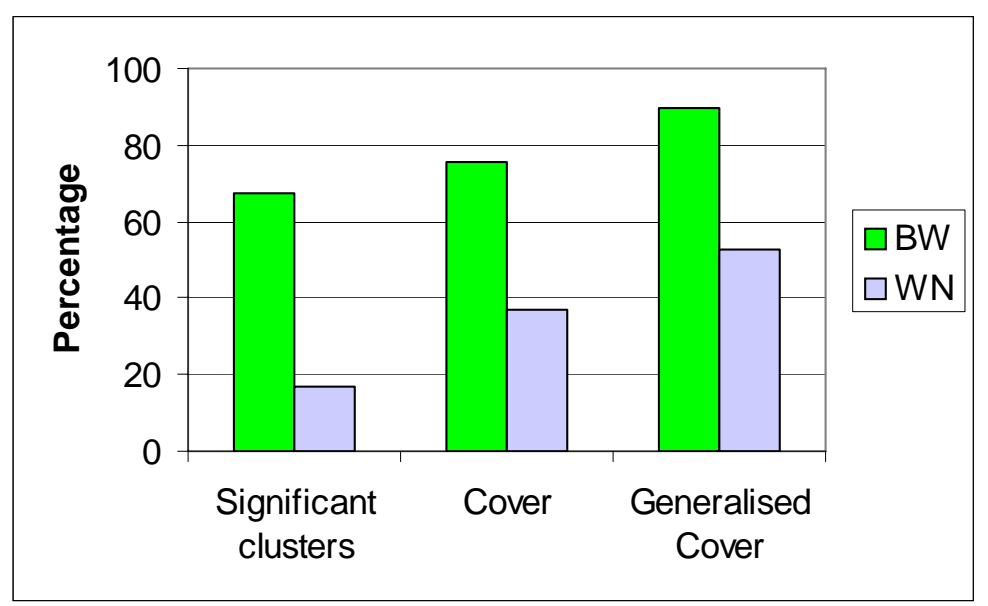

Figure 5.10: Average change to the total number of rules

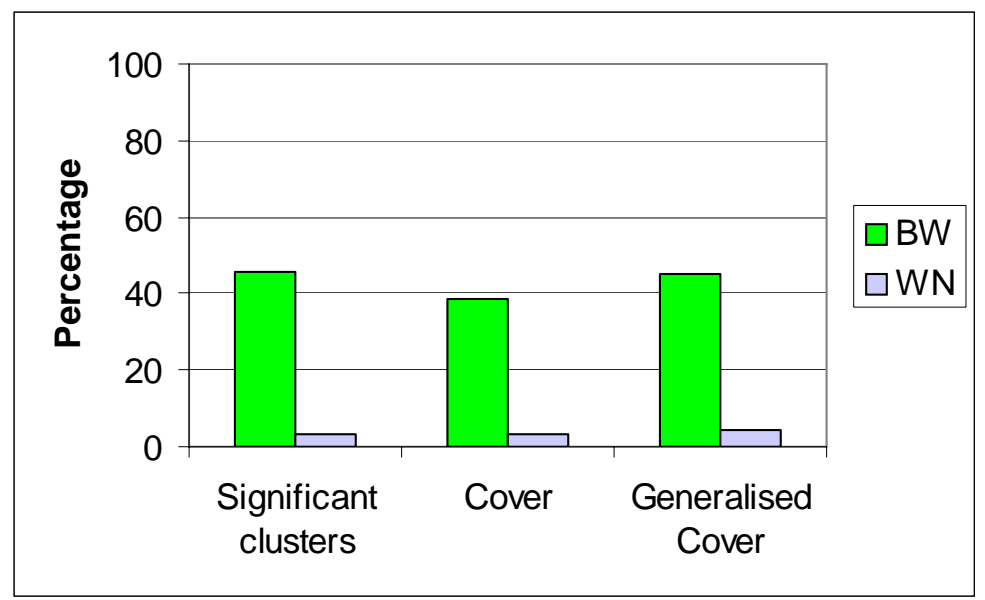

Figure 5.11: Average number of retained rules

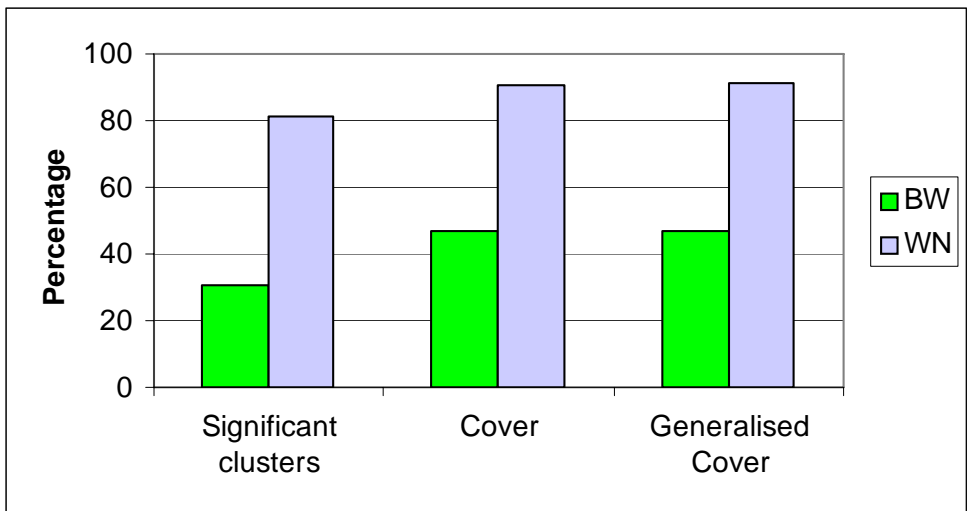

Figure 5.12: Average number of new rules 


\section{The rules' words}

Figures 5.13 to 5.15 show the changes to the number of rules' words when some descriptors are deleted from the descriptor sets of the instances. The figures show the changes to the total number of words, the number of retained words of the rules, and the number of new words.

When using BW or WN, on average, there are fewer rules' words after deleting descriptors than before deleting descriptors (figure 5.13). On average, there are more rules' words when using BW than when using WN; between $89 \%$ and $93 \%$ as many rules' words when using BW, but only between $46 \%$ and $50 \%$ as many rules' words when using WN.

When using BW or WN, on average, the significant cluster set has greater decrease, in the number of rules' words, than the cover cluster set, which in turn, has greater decrease than the generalised cover cluster set.

When clustering after deleting descriptors, on average, the rules' words retained from the clusters found when clustering before deleting descriptors, are many more when using BW than when using WN. Between $74 \%$ and $77 \%$ of rules' words are retained when using BW, but only between $21 \%$ and $25 \%$ of rules' words are retained when using WN. For BW, the significant cluster set retained more rules' words than the generalised cover cluster set, which in turn, retained more rules' words than the cover cluster set. In contrast, for WN, the significant cluster set retained more rules' words than the cover cluster set, which in turn, retained more rules' words than the generalised cover cluster set (figures 5.14).

When clustering after deleting descriptors, on average, the rules' words that are new found are fewer when using BW than when using WN. Between only $13 \%$ and $20 \%$ of the rules' words are new when using BW, but between $44 \%$ and $56 \%$ of the rules' words are new when using WN (figure 5.15). The significant cluster set has fewer rules' words that are new than the cover 
cluster set, which in turn, has fewer rules' words that are new than the generalised cover cluster set.

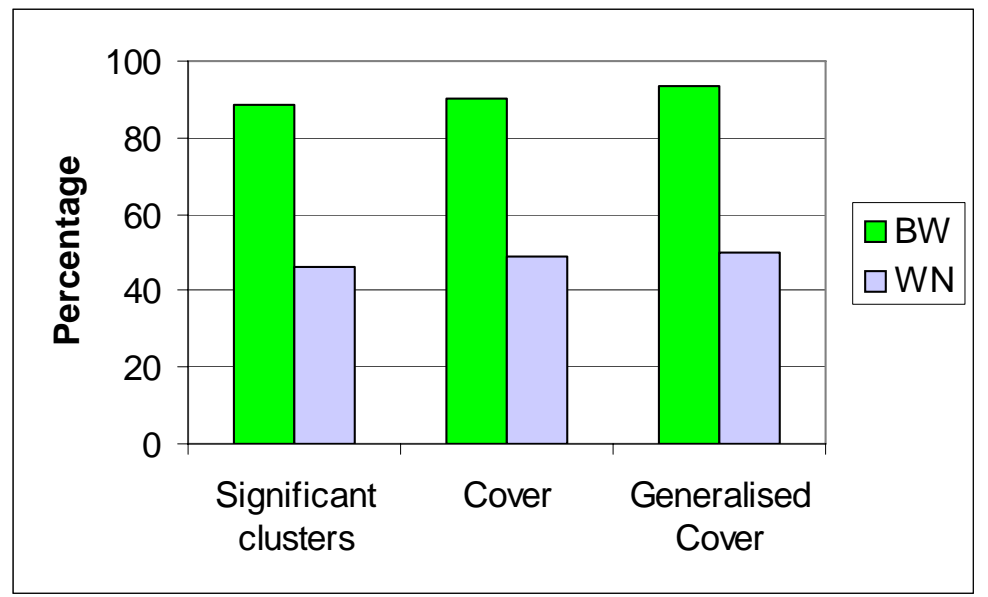

Figure 5.13: Average change to the total number of rules' words

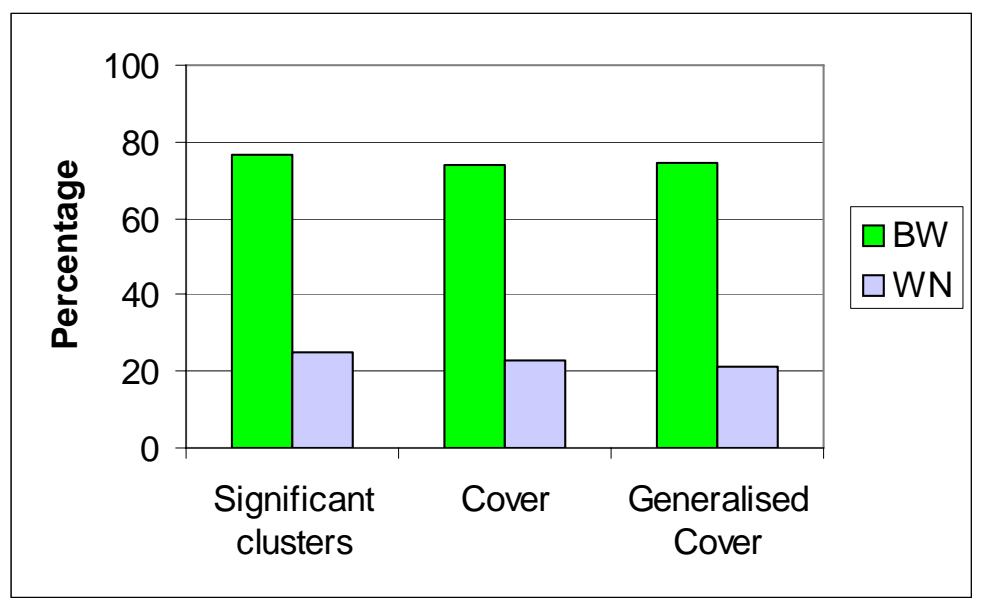

Figure 5.14: Average number of retained rules' words

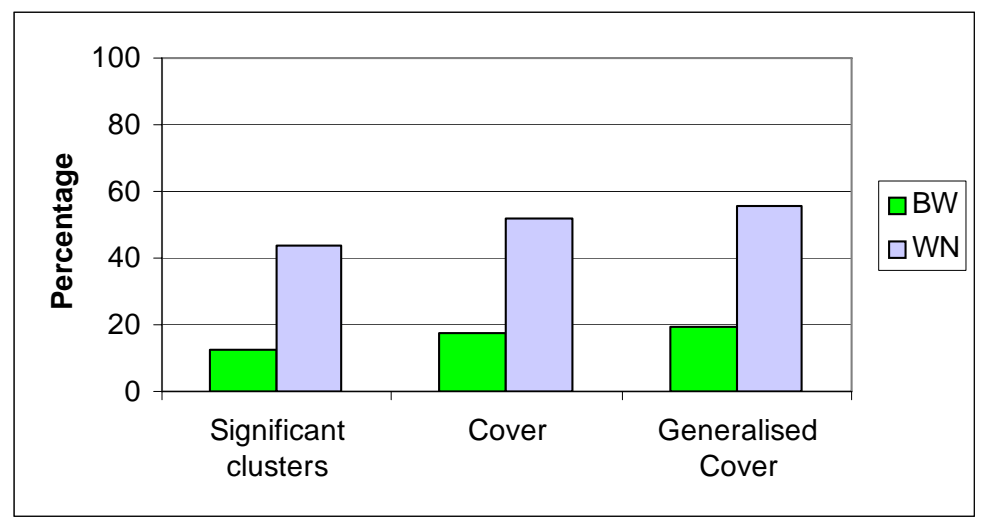

Figure 5.15: Average number of new rules' words 


\section{The words per rule}

Figure 5.16 shows the changes to the number of words per rule when some descriptors are deleted from the descriptor sets of the instances.

When using BW or WN, on average, there are fewer words per rule when clustering after deleting descriptors than when clustering before deleting descriptors. On average, there are more words per rule when using BW than when using WN. Between 96\% and 97\% as many words per rule when using BW, but only between $63 \%$ and $65 \%$ as many words per rule when using WN, compared with words per rule when clustering before deleting descriptors.

On average, for each of $\mathrm{BW}$ and $\mathrm{WN}$, the difference in change in the number of words per rule is very little between cluster sets.

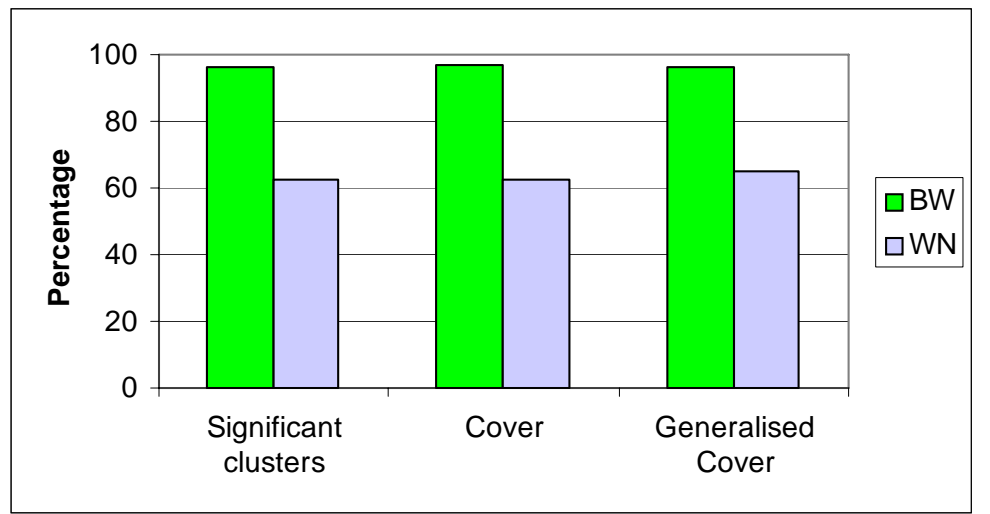

Figure 5.16: Average change to the number of words per rule 


\section{The execution time}

Figure 5.17 shows the changes to the clustering execution time when some descriptors are deleted from the descriptor sets of the instances.

When clustering after deleting descriptors using BW or WN, on average, the execution time is less than that when clustering before deleting descriptors. On average, the execution time, when clustering using BW, is more than that when using WN; about $74 \%$ as much time when using BW, but only about $11 \%$ as much time when using WN, compared with the execution time when clustering before deleting descriptors.

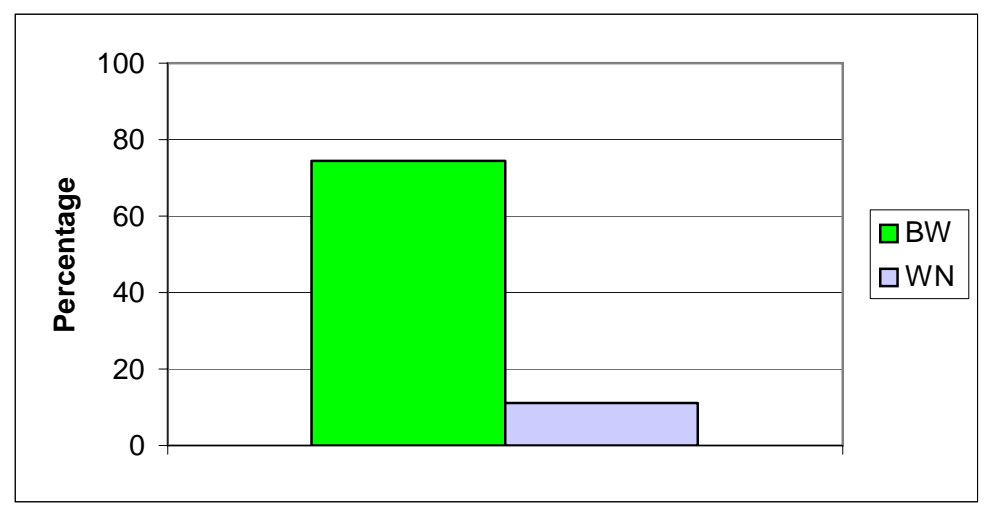

Figure 5.17: Average change to the clustering execution time 


\subsection{Analysis and discussion}

The following sections analyse and discuss the results shown in the results section.

\section{Clusters and instances}

Figure 5.2 shows that, on average, when clustering after deleting descriptors using BW, there is almost no change to the number of clusters found by MAXCCLUS, compared to that when clustering before deleting descriptors. The small loss in the number of significant clusters (about 3\%) does not have an important effect on the number of instances that MAXCCLUS can describe (figure 5.7). When clustering after deleting descriptors using BW, MAXCCLUS is able to describe almost the same number of instances as when clustering before deleting descriptors. However, MAXCCLUS needs less execution time for clustering (figure 5.17): on average, MAXCCLUS needs only about $75 \%$ of the time that it needs for clustering before deleting descriptors.

The reduction in execution time MAXCCLUS needs to cluster instances is because of the reduction in the sizes of the descriptor sets of the instances using BW. MAXCCLUS performs clustering by creating clusters of instances depending on the descriptor sets of these instances. Therefore, when there are fewer descriptors there will be fewer combinations of descriptors to create the clusters of these combinations. Although figure 5.2 showed that the significant cluster set did not change much, the total number of clusters created (the set of all-clusters) was much smaller. This reduces the time required for the permutation test. Note that the clusters that were lost were not significant clusters.

In contrast, when clustering after deleting descriptors using $\mathrm{WN}$, the observations mentioned in the previous sections become more noticeable. There is a large reduction in the number of clusters, especially the significant 
cluster set (figure 5.2). This reduction in the number of clusters is accompanied by a large reduction in the number of instances that MAXCCLUS can describe (figure 5.7). Although the execution time reduces dramatically to an average of $11 \%$, using WN is not preferable because of the loss in the number of instances that MAXCCLUS is able to describe.

\section{Rules and rules' words}

Figure 5.10 shows that, on average, when clustering after deleting descriptors using BW or WN, MAXCCLUS finds fewer rules than when clustering before deleting descriptors. On the other hand, figure 5.13 shows that the number of rules' words reduces too, but not as much as the number of rules. The reduction of the number of rules and the number of rules' words is due to the reduction of the sizes of descriptor sets of the instances because of deleting descriptors from these sets. The smaller change for BW than for WN is because of the smaller reduction of the sizes of the descriptor sets of the instances when using BW than when using WN.

Figure 5.4 shows that there are clusters of instances from clustering before deleting descriptors that are subsumed by new clusters from clustering after deleting descriptors (a considerable number of significant clusters when using BW). The subsumed clusters found by MAXCCLUS represent sets of instances that MAXCCLUS clustered using non-domain (uninformative) descriptors. When clustering after deleting descriptors, the instances that belong to the subsumed clusters, lack the non-domain words; therefore MAXCCLUS clusters these instances using their descriptors that are now more domain-relevant. Therefore MAXCCLUS finds clusters that describe more instances including the instances that belong to the subsumed clusters. These clusters that MAXCCLUS finds after deleting descriptors are bigger and scientifically more informative. 
By looking at the actual rules generated, we observed that words like: "as", "family", "it", "property", "step", "that", "this" are lost from the BW rules that characterise the clusters. Instead of these words, new words that are more domain-relevant appeared in the rules: "coli", "two-dimensional", "gel", "transcription", "transmembrane", "catalyze".

For example, figure 5.18 shows a cluster of instances before deleting descriptors. Using the same data set, after deleting descriptors using BW (figure 5.19), the same cluster lost the word "family" (which is in the biblewords dictionary and is not informative) from its Sufficient set of words. The loss of the word "family" made the word "belong" (which is not in the biblewords dictionary) become a word of the Necessary set of words instead of the Sufficient set of words, causing the Sufficient set of words to became empty. The effect of this was to reduce the number of rules from 2 to 1 in this example, which is a reduction of $50 \%$. It also made the remaining rule relatively more informative by not including the word "family" in it. This is because the word "family" is closely connected to the word "belong": they appear together in the textual database implying a relationship between them. Some of the examples of the relation between the words "family" and "belong" from the Swiss-Prot database textual data are:

"BELONGS TO THE TRANSALDOLASE FAMILY"

"BELONGS TO THE GHMP KINASE FAMILY"

"BELONGS TO THE SODIUM:ALANINE SYMPORTER FAMILY"

Note, the word "BELONGS" was stemmed to "belong" in the pre-processing.

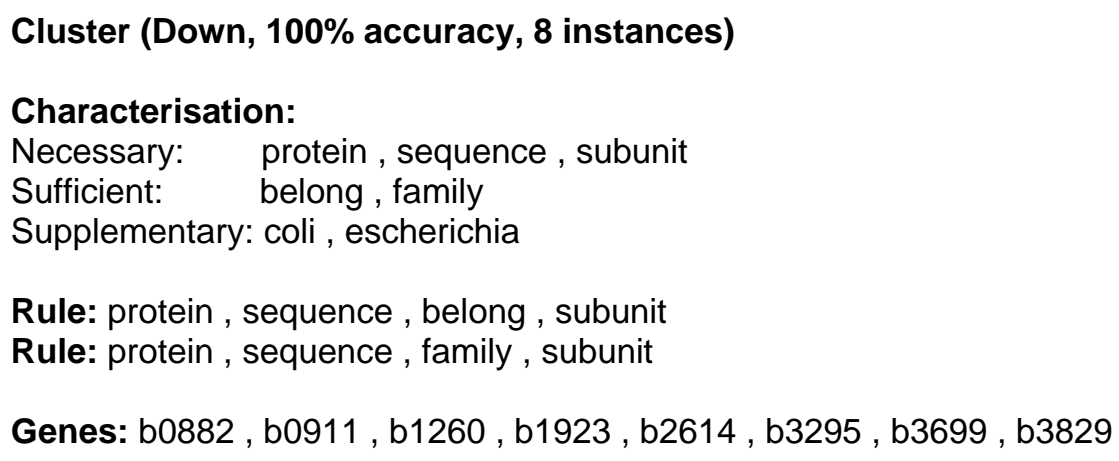

Figure 5.18: A cluster before deleting descriptors 


\section{Cluster (Down, 100\% accuracy, 8 instances)}

\section{Characterisation:}

Necessary: protein, sequence, belong, subunit

Supplementary: coli , escherichia

Rule: protein , sequence, belong, subunit

Genes: b0882 , b0911 , b1260 , b1923 , b2614 , b3295 , b3699 , b3829

Figure 5.19: The same cluster of figure 5.18, but after deleting descriptors using BW

In contrast, after deleting descriptors using WN, the same cluster of instances is lost; MAXCCLUS is not able to construct this cluster at all because of the severe loss of descriptors in the instances' descriptor sets.

Figures 5.3 to 5.5 show that clustering with $\mathrm{WN}$ generates a very different set of clusters, much more so than for BW. This is because the major change in the descriptor sets of the instances after deleting descriptors using $W N$, including many descriptors that are relevant to the domain. Losing lots of domain-relevant descriptors when using WN cause MAXCCLUS to find clusters that are missing important scientific knowledge (information). Therefore it seams that using WN is a bad thing to do, in spite the fact that the remaining words are the uncommon domain-relevant descriptors, which may lead one to expect a good result.

Some of the words that are retained when using BW are lost when using WN. Examples of these words are: "escherichia", "hypothetical", "salmonella", "nucleotide", "biosynthesis", "expression", "cytoplasmic". These are common domain-relevant words.

Instead of the lost words, when clustering using WN, new words appear in the rules. Examples of these new words are: "ec@2", "kda", "acetyl-coa", 
"transmembrane", "atp-binding", "dna-binding", "phosphoenolpyruvate", "allosteric", "3-phosphate", "homotetramer", "synthetase", "I-glutamate". These are less common domain-relevant words than the words in the previous paragraph.

Figure 5.20 shows an example from another data set, of a cluster after deleting descriptors using WN. The word "phosphoenolpyruvate", which is the only word in the Necessary set of words, does not exist in WordNet, also it does not exist in any word set of any cluster when clustering before deleting descriptors or when clustering after deleting descriptors using BW. This cluster contains only one rule that has only one word, which is an uncommon domain-relevant word.

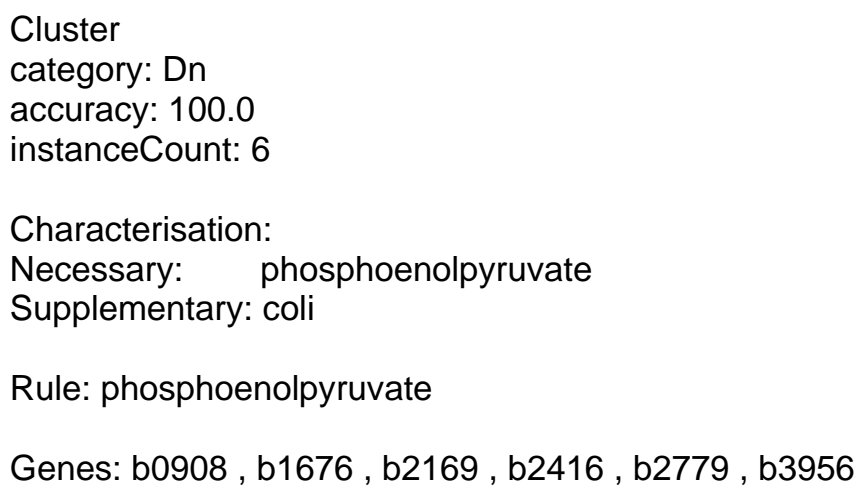

Figure 5.20: A cluster after deleting descriptors using WN 


\subsection{Conclusion}

Deleting the non-domain descriptors in the Bible-words dictionary improves the rules that characterise the clusters created by MAXCCLUS, making the rules more scientifically informative. MAXCCLUS is still able to describe almost the same number of instances compared with what it can cover without deleting descriptors. Also, deleting the non-domain descriptors reduces the clustering time.

However, deleting more descriptors, including common domain-relevant descriptors, using the WordNet dictionary gives bad results, because MAXCCLUS is not able to describe as many instances or create as many clusters as before deleting descriptors.

\subsection{Recommendations}

These experiments demonstrate that it is advantageous to delete descriptors that are not relevant to the applied domain, because they do not carry any significant scientific information related to the domain. On the other hand, it is important not to delete too many descriptors, especially domain-relevant ones, because it removes essential information needed for the clustering process. 


\section{Chapter 6}

\section{Adding Descriptors}

This chapter describes a clustering experiment designed to study the effects on MAXCCLUS clustering after adding more descriptors to the descriptor sets of the instances, and comparing its results with the reference clustering experiment where no descriptors are added. The added descriptors are the synonyms and hypernyms of the already existing descriptors. We expected this to help MAXCCLUS to form clusters of instances that have semantic relationships but were not previously clustered because they did not share any literal descriptors. Unexpectedly, the result of this chapter is to show that adding descriptors does not improve MAXCCLUS clustering; MAXCCLUS is not able to describe more instances and it generates the clusters much more slowly.

\subsection{The purpose of the experiment}

Some genes can have different descriptor sets and they may not share any descriptor, although some of these descriptors have a very similar meaning. For example, suppose that there are two instances that do not share any descriptor. If the first instance has the word "expand" as one of its descriptors, and the second instance has the word "enlarge" as one of its descriptors, then these instances may belong to the same cluster because the words "expand" and "enlarge" have the same meaning. In this case, we expect that adding 
synonyms will enable MAXCCLUS to describe more instances by being able to see the similarity in their descriptor sets.

\subsection{Adding descriptors}

We chose three approaches to add more descriptors:

- adding synonyms ( $\mathrm{x}$ is a synonym of $\mathrm{y}$, if $\mathrm{x}$ and $\mathrm{y}$ have the same meaning). An example: the word "enlarge" is a synonym of the word "expand".

- adding hypernyms ( $x$ is a hypernym of $y$, if $y$ is a kind of $x$ ). An example: the word "activity" is a hypernym of the word "play".

- and adding both synonyms and hypernyms

To ensure that any descriptors we add are relevant to the textual data set, we only add a word that is a synonym or hypernym of a descriptor if that word already exists in the set of all descriptors (which is the union of the descriptor sets of all instances). This project uses WordNet to find synonyms and hypernyms of a descriptor.

\subsubsection{WordNet}

WordNet is a lexical database for the English language from Princeton University [Princeton University, 2003]. WordNet contains 144,309 of the open class words (nouns, verbs, adjectives and adverbs) in English. We created a program using the Perl computer-programming language, which uses a special module [Rennie, J. 2002] to access the WordNet database to retrieve all synonyms and hypernyms of descriptors. 


\subsubsection{The algorithm for adding descriptors}

Algorithm 6.1 (figure 6.1) attempts to increase the size of the descriptor set of each instance in the descriptor table file (the input to this algorithm) by going through each descriptor in an instance's descriptor set, and attempting to retrieve the relevant senses of this descriptor from WordNet (if any senses exist). In WordNet, the senses of a word include the synonyms and hypernyms of the word. For each synonym or hypernym retrieved, it checks whether the word exists in the set of all-descriptors, and then adds the word to this instance's descriptor set. The set of all-descriptors is the union of the descriptor sets of all instances. The algorithm writes each instance's new descriptor set to the new descriptor table file, which MAXCCLUS will use in this chapter's clustering experiment.

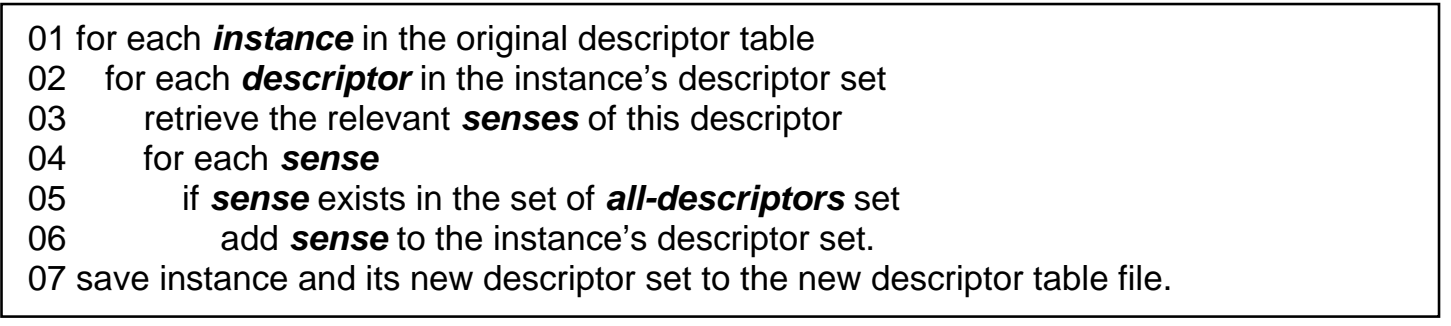

Figure 6.1: Algorithm 6.1: Adding senses to instances' descriptor sets.

Figure 6.2 shows the increase in the average size of the descriptor sets for the three cases. Adding hypernyms gives in lowest increase value, on the other hand, adding synonyms and hypernyms together gives in the highest increase value.

\begin{tabular}{|l|c|}
\hline Added senses & Increase \\
\hline Synonyms & $69 \%$ \\
\hline Hypernyms & $50 \%$ \\
\hline $\begin{array}{l}\text { Synonyms and } \\
\text { Hypernyms }\end{array}$ & $117 \%$ \\
\hline
\end{tabular}

Figure 6.2: The increase in the average size of the descriptor sets for the three cases. 


\subsection{Experimental results}

This section presents the results of running MAXCCLUS on 23 of the microarray experimental data sets (data set: $3,6,7,8,10,11,12,13,16,18$, $20,21,22,23,24,25,27,28,29,30,35,36$, and 40) first after adding synonyms, second, after adding hypernyms, and third after adding both synonyms and hypernyms. We will refer to these three sets of results by $\boldsymbol{S} Y$, $\boldsymbol{H Y}$, and $\mathbf{S H}$ respectively in the rest of the chapter. This section also compares these results with each other and with the results produced by the reference clustering experiment (the one before adding descriptors). Figures 6.3 to 6.18 show the results for the number of clusters, the number of instances, the number of rules, the number of words in the rules, the number of words per rule, and the execution time.

\section{The clusters}

Figures 6.3 to 6.6 show the changes to the number of clusters when more descriptors are added to the descriptor sets of the instances. The figures show the changes to the total number of clusters, the number of retained clusters, the number of clusters subsumed by new clusters, and the number of new clusters.

When using SY, HY or $\mathrm{SH}$, on average, there are fewer clusters in the significant cluster set when clustering after adding descriptors than when clustering before adding descriptors, but there are more clusters in the cover and the generalised cover cluster sets (figure 6.3).

For the significant cluster set and the generalised cover cluster set, on average, there are more clusters when using $\mathrm{HY}$ than when using $\mathrm{SH}$, which in turn, there are more clusters than when using SY. For the cover cluster set, there are more clusters when using SH than when using HY, which in turn, there more clusters than when using SY. 
When clustering after adding descriptors, on average, the clusters retained from the clusters found when clustering before adding descriptors, are more when using SY than when using $\mathrm{HY}$, which in turn has more retained clusters than when using $\mathrm{SH}$. Between $26 \%$ and $36 \%$ of clusters are retained when using SY, between $14 \%$ and $21 \%$ of clusters are retained when using $\mathrm{HY}$, but only between $8 \%$ and $14 \%$ of clusters are retained when using SH. For SY, $\mathrm{HY}$ and $\mathrm{SH}$, the significant cluster set retained more clusters than the cover cluster set or the generalised cover cluster set (figures 6.4).

When clustering after adding descriptors, on average, the new clusters found are fewer when using SY than when using HY, which in turn has fewer new clusters than when using $\mathrm{SH}$. Only between $59 \%$ and $74 \%$ of clusters are new when using SY; between $75 \%$ and $85 \%$ of clusters are new when using $\mathrm{HY}$, and between $84 \%$ and $91 \%$ of clusters are new when using SH (figure 6.6).

For the significant cluster set, on average, more clusters found from clustering before adding descriptors, are subsumed by new clusters found from clustering after adding descriptors using $\mathrm{HY}$ than that using $\mathrm{SH}$, which in turn has more clusters subsumed than when using SY. For the cover cluster set, on average, more clusters are subsumed using $\mathrm{SY}$ than that using $\mathrm{SH}$, which in turn has more clusters subsumed than when using HY. For the generalised cover cluster set, on average, more clusters are subsumed using HY than that using SY, which in turn has more clusters subsumed than when using $\mathrm{SH}$. The significant cluster set has more clusters subsumed than the generalised cover cluster set, which in turn has more clusters subsumed than the cover cluster set (figure 6.5). 


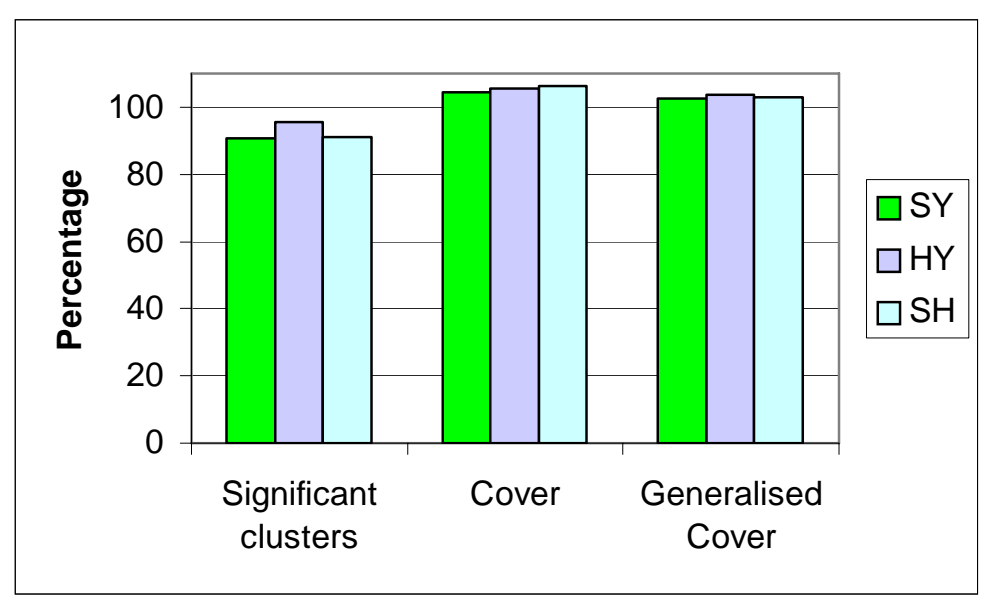

Figure 6.3: Average change to the total number of clusters

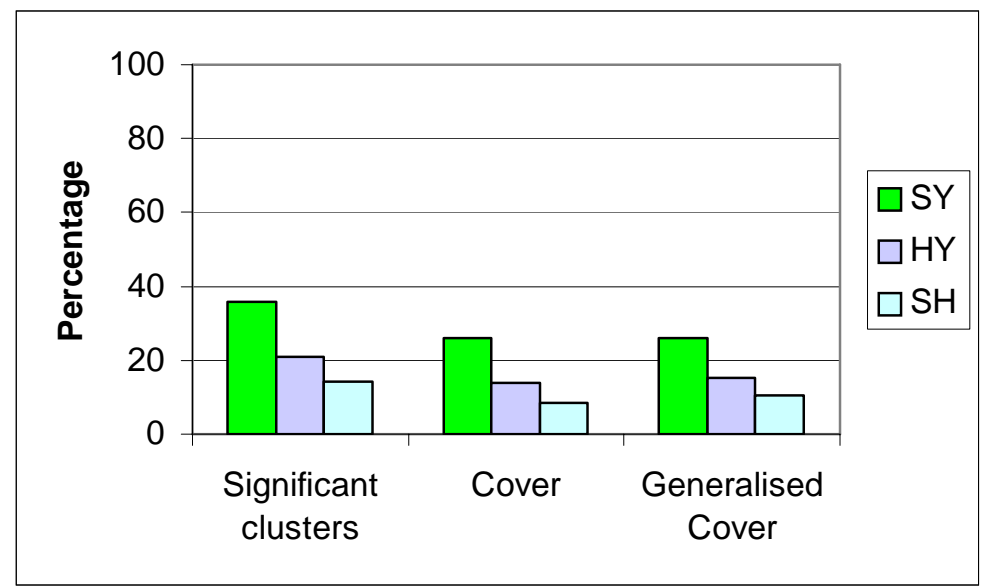

Figure 6.4: Average number of retained clusters

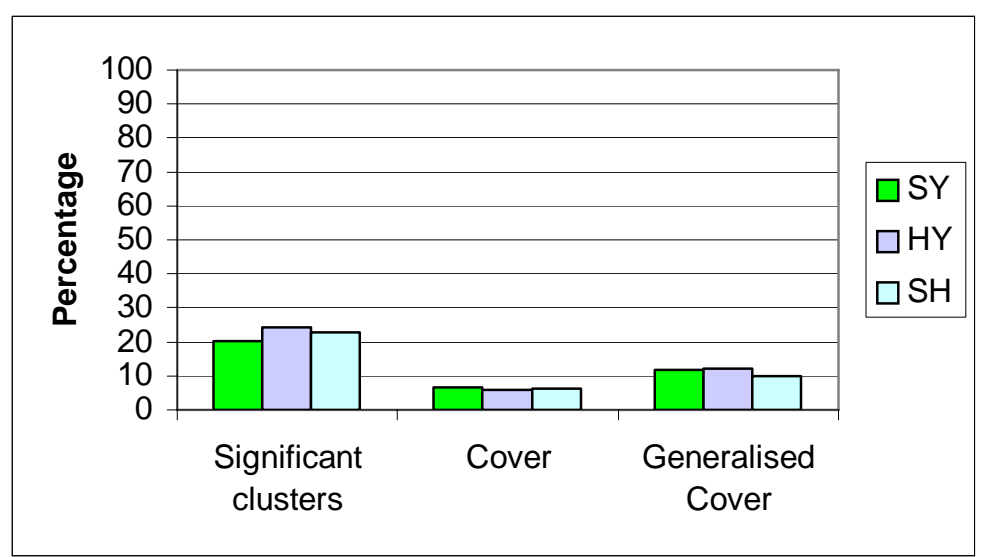

Figure 6.5: Average number of clusters subsumed by new clusters 


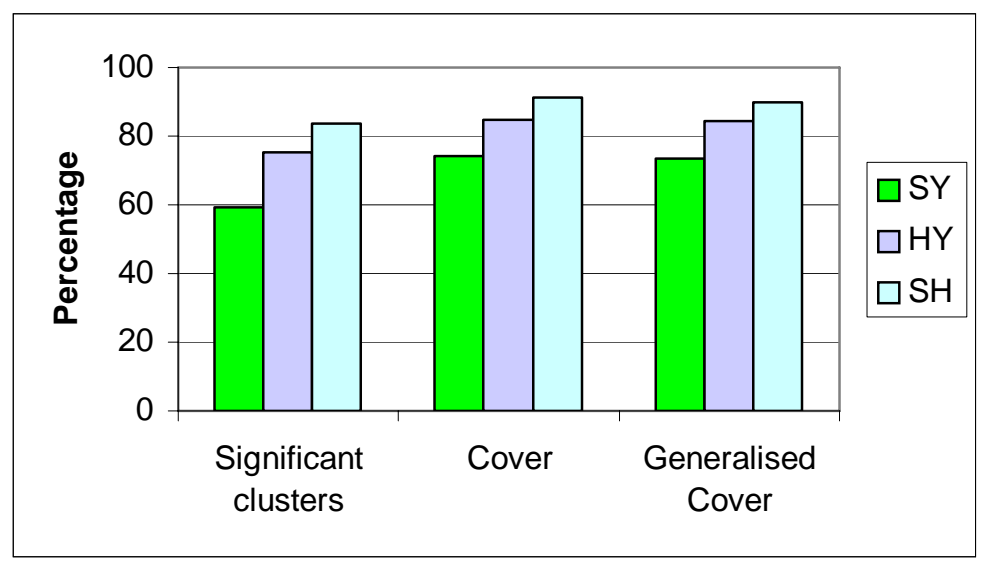

Figure 6.6: Average number of new clusters

\section{Significant clusters minimum size}

Figure 6.7 shows the changes to the significant clusters' minimum size for each category when more descriptors are added to the descriptor sets of the instances.

When clustering after adding descriptors using $\mathrm{SY}, \mathrm{HY}$, or $\mathrm{SH}$, on average, the significant clusters' minimum size for each category is more than that when clustering before adding descriptors. On average, the increase in the significant clusters' minimum size, when using SY, is less than that when using $\mathrm{HY}$, which in turn is less than that when using $\mathrm{SH}$. When using any of $\mathrm{SY}, \mathrm{HY}$, or $\mathrm{SH}$, the increase in the significant clusters' minimum size for the Up category is more than that for the Down category.

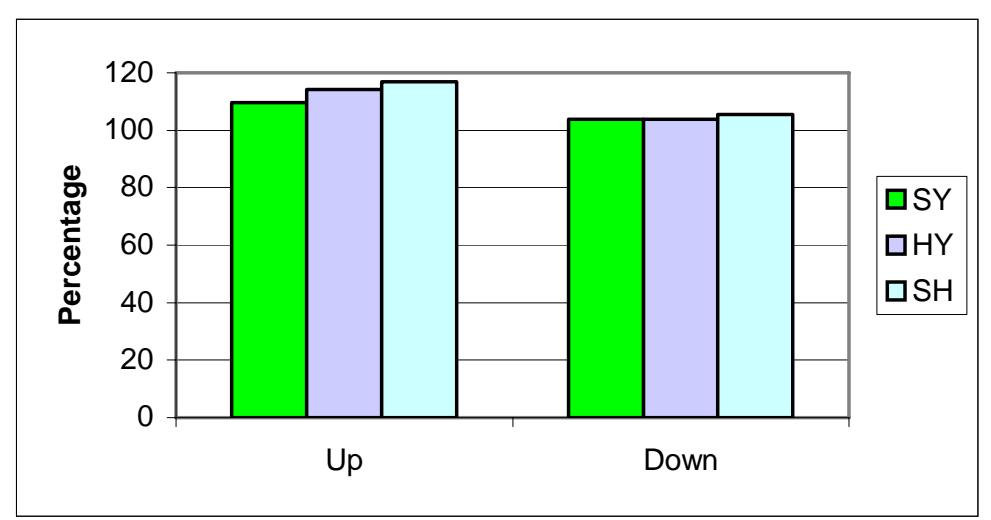

Figure 6.7: Average change to the significant clusters' minimum size for each category 


\section{The instances}

Figures 6.8 to 6.10 show the changes to the number of instances when more descriptors are added to the descriptor sets of the instances. The figures show the changes to the total number of instances, the number of retained instances, and the number of new instances.

In each figure, the results of the significant and the cover cluster sets are the same because both sets should cover the same instances.

When using SY, HY or $\mathrm{SH}$, on average, there are fewer instances in the significant, cover, and generalised cover cluster sets after adding descriptors than before adding descriptors, with almost no change in the number of instances in the significant cluster set using SY (figure 6.8).

For any of the cluster sets, significant, cover, or generalised cover cluster set, when using SY, on average, there are more instances than when using $\mathrm{HY}$ or $\mathrm{SH}$.

When clustering after adding descriptors, on average, the instances retained from the clusters found when clustering before adding descriptors, are more when using SY than when using $\mathrm{HY}$, which in turn has more retained instances than when using $\mathrm{SH}$. About $89 \%$ of clusters are retained when using SY, about $85 \%$ of instances are retained when using HY, but only about $83 \%$ instances are retained when using $\mathrm{SH}$. For $\mathrm{SY}, \mathrm{HY}$ and $\mathrm{SH}$, the generalised cover cluster set retained fewer instances than the significant cluster set or the cover cluster set (figures 6.9).

When clustering after adding descriptors, on average, the new instances found are fewer when using SY than when using HY, which in turn has fewer new instances than when using $\mathrm{SH}$. Only about $8 \%$ of instances are new when using SY; about $9 \%$ of instances are new when using $\mathrm{HY}$, and about $11 \%$ of instances are new when using $\mathrm{SH}$ (figure 6.10). 


\section{Chapter 6: Adding Descriptors}

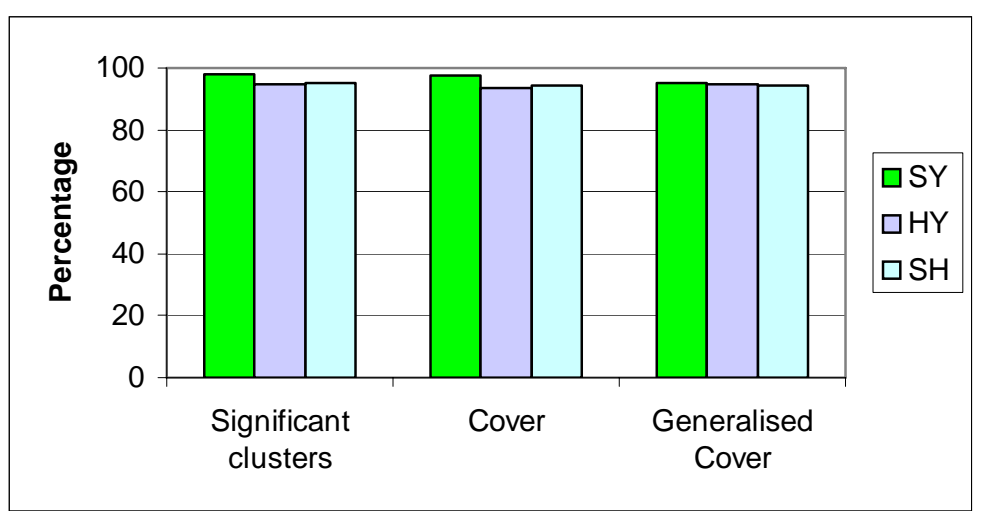

Figure 6.8: Average change to the total number of instances

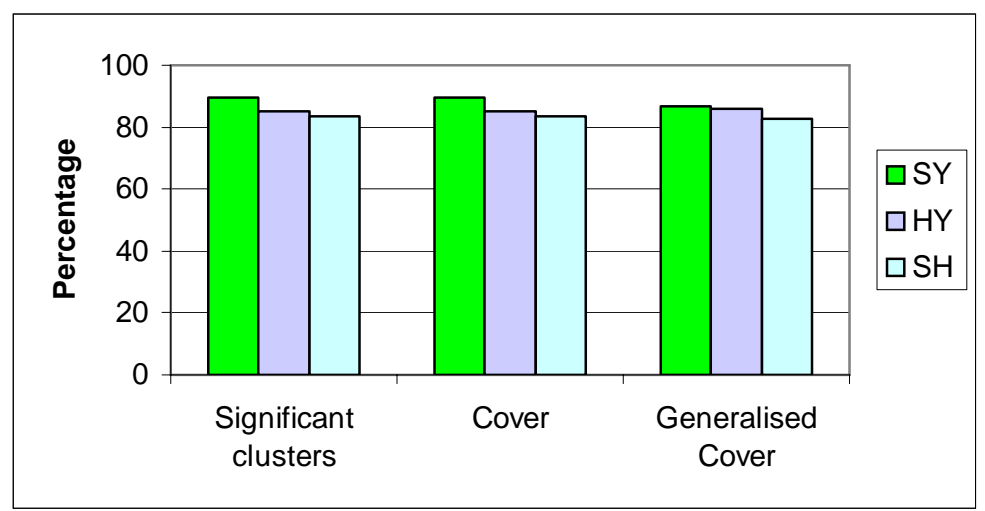

Figure 6.9: Average number of retained instances

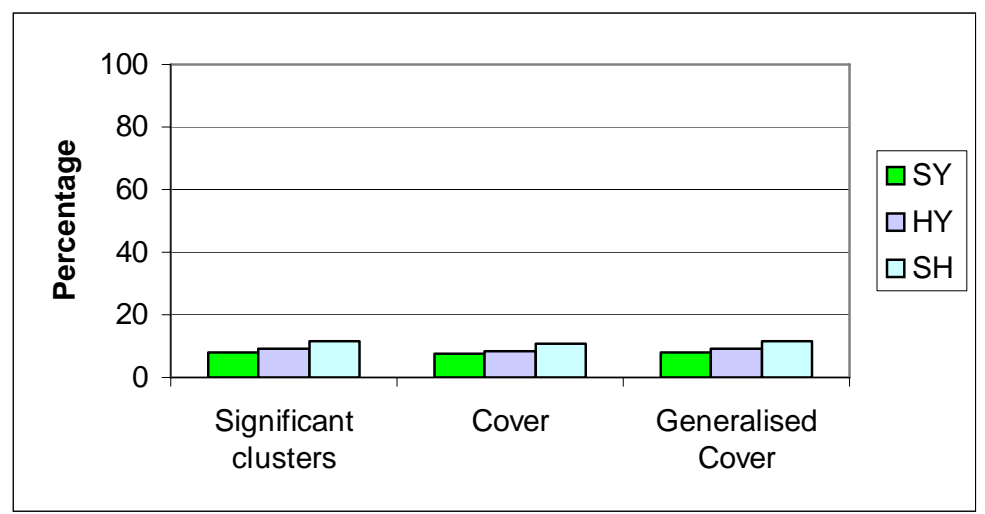

Figure 6.10: Average number of new instances 


\section{The rules:}

Figures 6.11 to 6.13 show the changes to the number of rules when more descriptors are added to the descriptor sets of the instances. The figures show the changes to the total number of rules, the number of retained rules, and the number of new rules.

When using SY, HY or SH, on average, there are many more rules when clustering after adding descriptors than when clustering before adding descriptors. On average, there are many more rules when using $\mathrm{SH}$ than when using SY, which in turn there are more rules than when using $\mathrm{HY}$ (figure 6.11).

The significant cluster set has a greater increase in the number of rules than the cover cluster set, which in turn has a greater increase than the generalised cover cluster set.

When clustering after adding descriptors, on average, the number of rules retained from the rules found when clustering before adding descriptors, is greater when using SY than when using HY, which in turn has more retained rules than when using $\mathrm{SH}$. Between $26 \%$ and $43 \%$ of rules are retained when using SY, between $14 \%$ and $27 \%$ of rules are retained when using $\mathrm{HY}$, but only between $7 \%$ and $17 \%$ of rules are retained when using SH. For SY, HY and $\mathrm{SH}$, the significant cluster set retained more rules than the cover cluster set, which returned more rules than the generalised cover cluster set (figures $6.12)$.

When clustering after adding descriptors, on average, the new rules found are more when using $\mathrm{SH}$ than when using SY or HY. Almost all the rules are new when using $\mathrm{SH}$, especially for the significant and the cover cluster sets (figure $6.13)$. 
On average, almost no rules found from clustering before adding descriptors, are subsumed by new rules found from clustering after adding descriptors using any of $\mathrm{SY}, \mathrm{HY}$ or $\mathrm{SH}$.

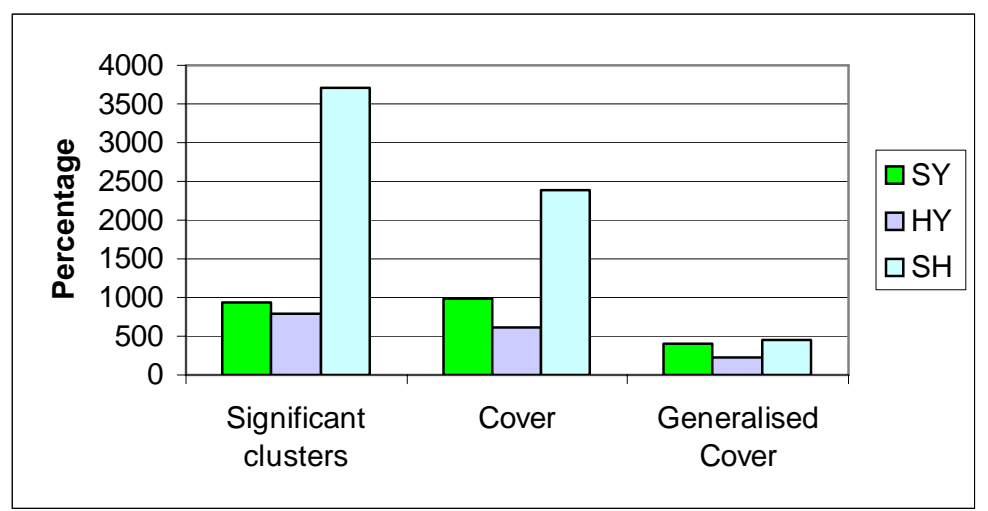

Figure 6.11: Average change to the total number of rules

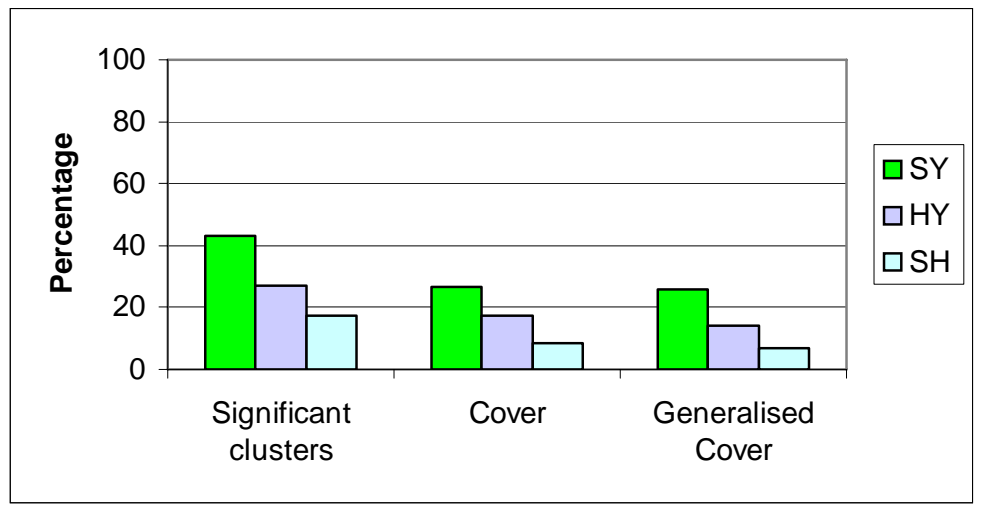

Figure 6.12: Average number of retained rules

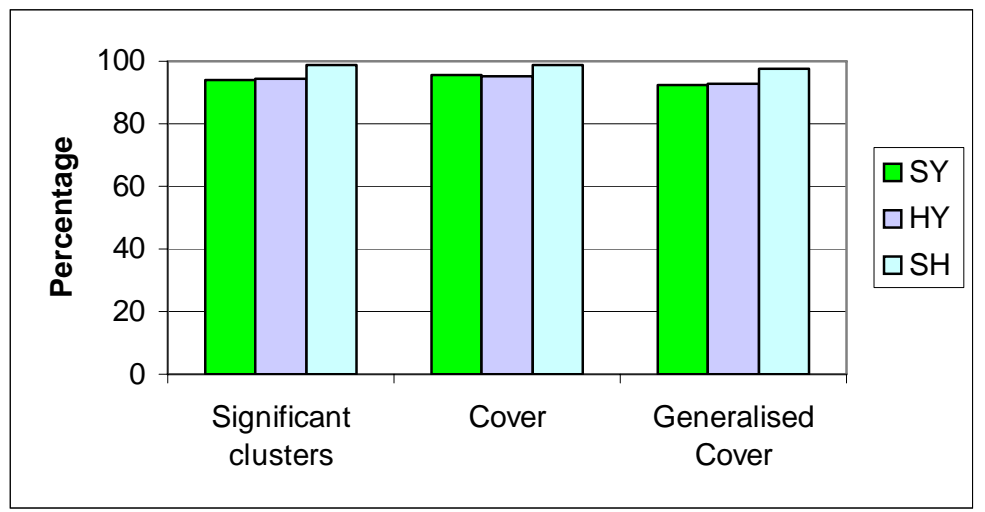

Figure 6.13: Average number of new rules 


\section{The rules' words}

Figures 6.14 to 6.16 show the changes to the number of rules' words when more descriptors are added to the descriptor sets of the instances. The figures show the changes to the total number, the number of retained words of the rules, and the number of new words.

On average, there are more rules' words after adding descriptors than before adding descriptors. When using $\mathrm{SH}$ there are more rules' words than when using SY, which in turn there are more rules words than when using HY. The cover cluster set has greater increase in the number of rules' words than the significant cluster set, which in turn, has greater increase than the generalised cover cluster set (figure 6.14).

When clustering after adding descriptors, on average, the number of rules' words retained from the clusters found when clustering before adding descriptors, is greater when using SY than when using $\mathrm{HY}$, which in turn has more retained rules' words than when using SH. Between $67 \%$ and $83 \%$ of rules' words are retained when using SY, between $57 \%$ and $73 \%$ of rules' words are retained when using HY, but only between $49 \%$ and $69 \%$ of rules' words are retained when using $\mathrm{SH}$. For $\mathrm{SY}, \mathrm{HY}$ and $\mathrm{SH}$, the significant cluster set retained more rules' words than the cover cluster set, which in turn retained more rules' words than the generalised cover cluster set (figures 6.15).

When clustering after adding descriptors, on average, the new rules' words found are fewer when using HY than when using SY, which in turn has fewer new rules' words than when using SH. Only between $51 \%$ and $57 \%$ of rules' words are new when using HY, between $52 \%$ and $57 \%$ of rules' words are new when using SY, and between $66 \%$ and $71 \%$ of rules' words are new when using $\mathrm{SH}$. The significant cluster set has fewer new rules' words than the generalised cover set, which in turn has fewer new rules' words than the cover cluster set (figure 6.16). 


\section{Chapter 6: Adding Descriptors}

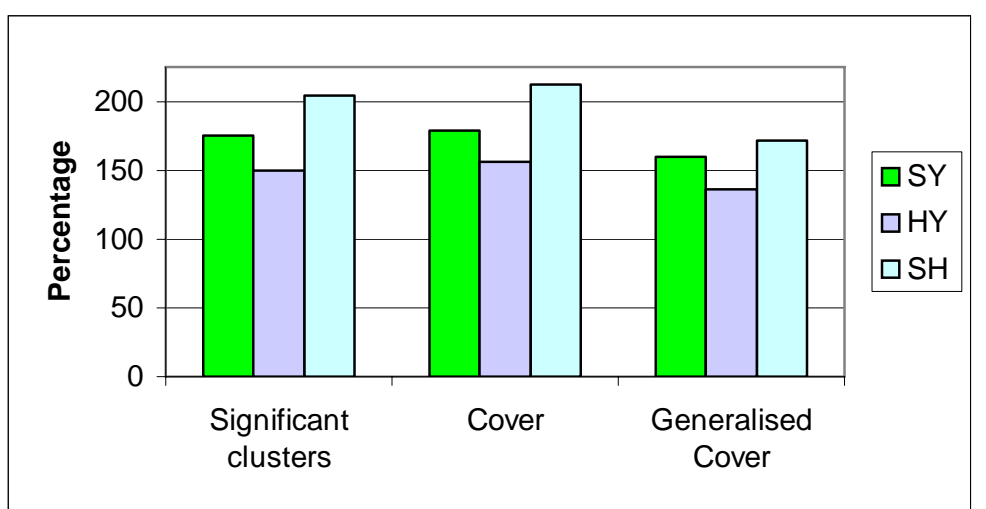

Figure 6.14: Average change to the total number of rules' words

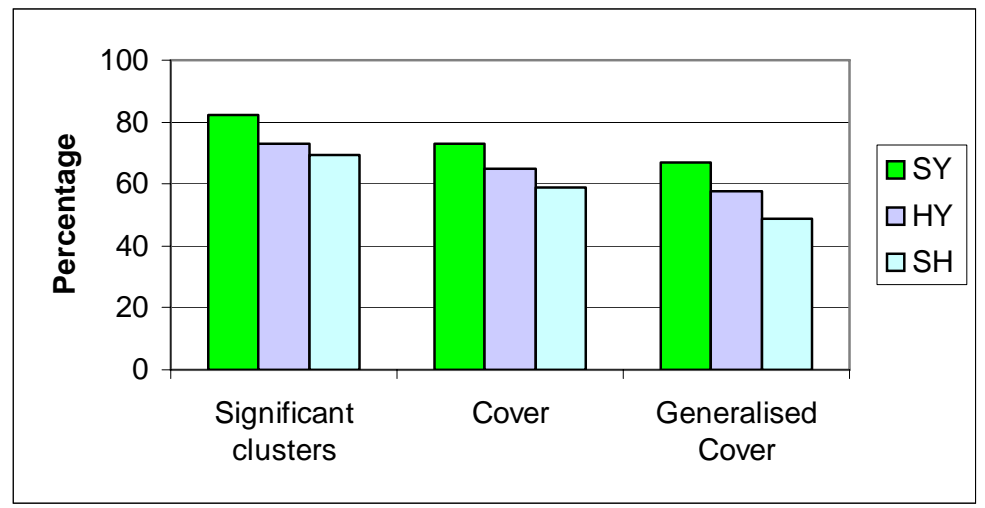

Figure 6.15: Average number of retained rules' words

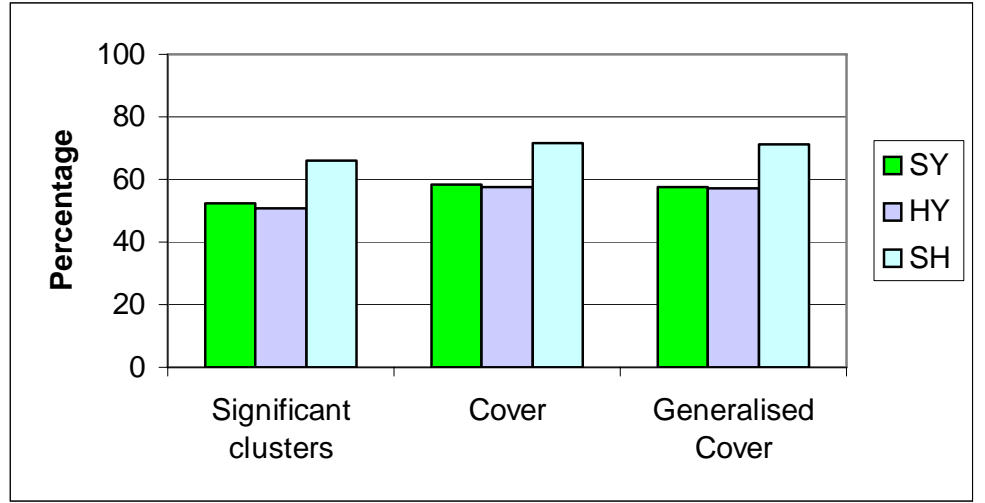

Figure 6.16: Average number of new rules' words 


\section{The words per rule}

Figure 6.17 shows the changes to the total number of words per rule when more descriptors are added to the descriptor sets of the instances.

On average, there are more words per rule when clustering after adding descriptors than when clustering before adding descriptors. On average, when using SY there are fewer words per rule than when using HY, which in turn generates fewer rules than when using $\mathrm{SH}$.

On average, when using SY or HY, the cover cluster set has greater increase, in the number of words per rule, than the generalised cover cluster set, which in turn, has greater increase than the significant cluster set. In contrast when using $\mathrm{SH}$, the cover cluster set has greater increase, in the number of words per rule, than the significant cluster set, which in turn, has greater increase than the generalised cover cluster set.

The statistical distributions in the figure show that almost all the data sets have more words per rule when clustering after adding descriptors than when clustering before adding descriptors. 


\section{Chapter 6: Adding Descriptors}

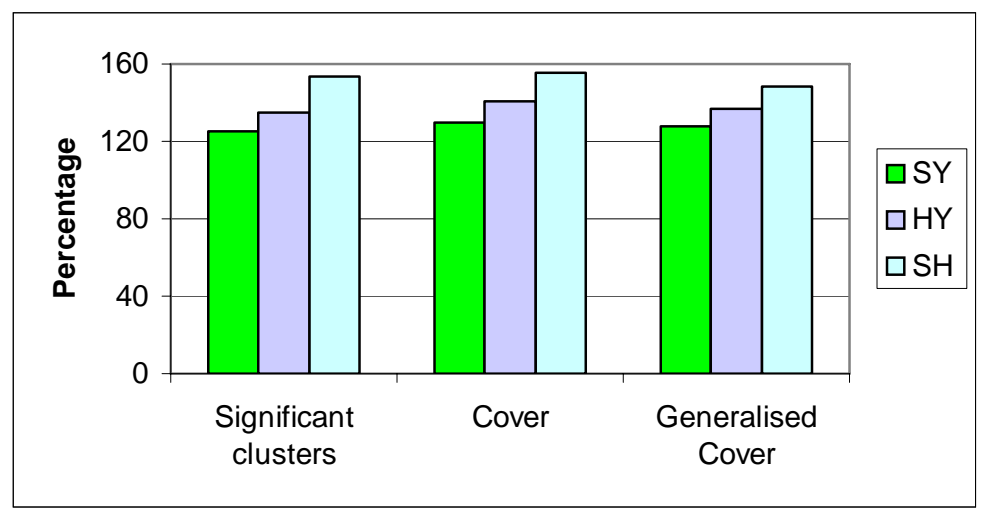

a: Average

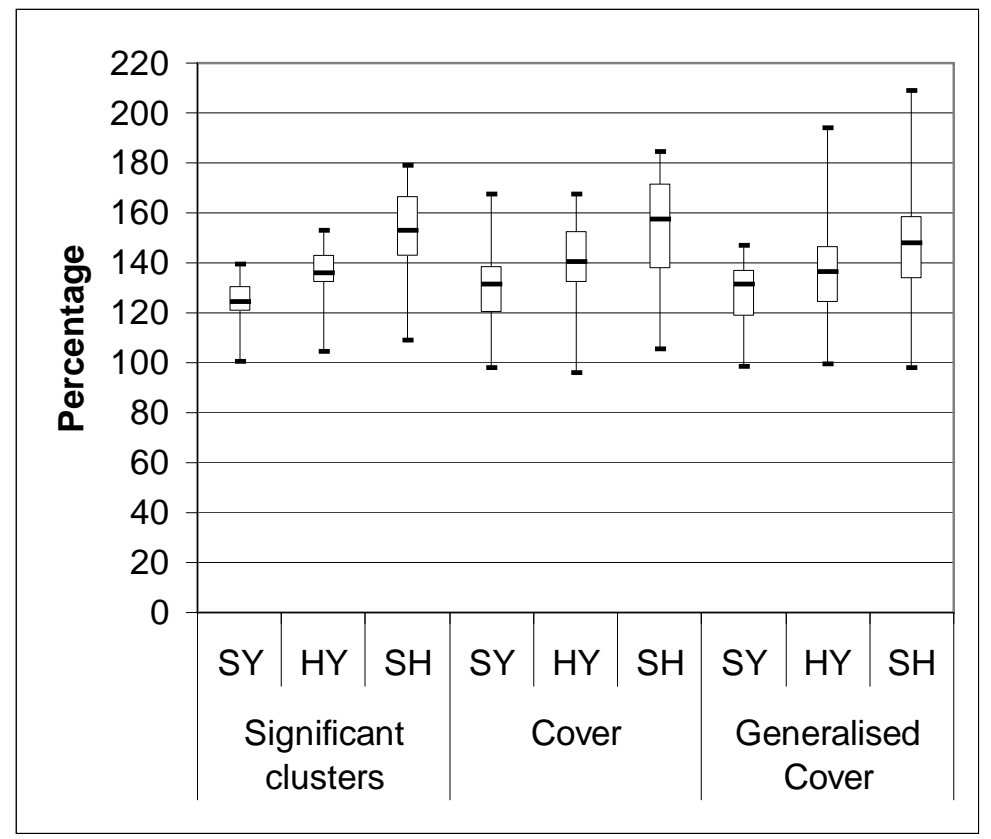

b: Distributions

Figure 6.17: Change to the number of words per rule 


\section{The execution time}

Figure 6.18 shows the changes to the clustering execution time when more descriptors are added to the descriptor sets of the instances.

When clustering after adding descriptors using SY, HY or SH, on average, the execution time is more than that when clustering before adding descriptors. On average, the execution time, when clustering using $\mathrm{SH}$, is more than that when using $\mathrm{HY}$, which in turn is more than that when using SY. About 7.8 times longer when using $\mathrm{SH}$, but only about 2.8 times longer when using $\mathrm{HY}$, and only about 2.6 times longer when using SY, compared with the execution time when clustering before adding descriptors.

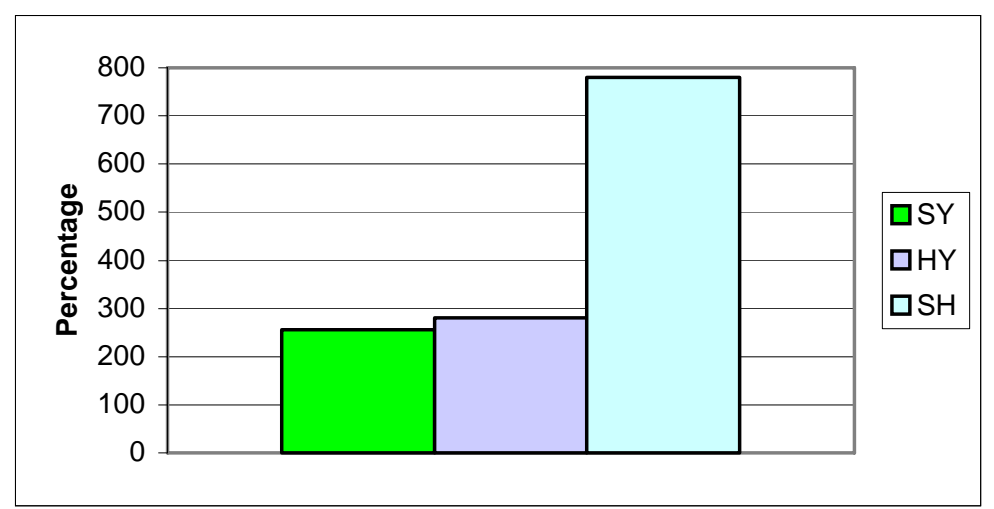

Figure 6.18: Average change to the clustering execution time 


\subsection{Analysis and discussion}

The following sections analyse and discuss the results shown in the results section.

\section{Clusters}

On average, there are fewer clusters in the significant cluster set after adding descriptors than before adding descriptors (figure 6.3). Although there is not a great reduction in the number of the significant clusters, the significant clusters when clustering after adding descriptors are quite different from when clustering before adding descriptors: there is a relatively low percentage of the retained significant clusters (figure 6.4). Noticing the relative high percentage of the subsumed significant clusters in the new ones (figure 6.5), this means that some of the new significant clusters, when clustering after adding descriptors, are bigger by subsuming other significant clusters from clustering before adding descriptors.

On average, after adding descriptors, the minimum size for significant clusters increased (figure 6.7). This means that some of the clusters from before adding descriptors are no longer considered significant.

This decrease in the number of significant clusters was a surprise, particularly given that the size of the set of all-clusters increased (from an average of about 43,000 clusters, before adding descriptors, to 71,000 for SY, 81,000 for $\mathrm{HY}$, and 96,000 for $\mathrm{SH}$ after adding descriptors). Usually for a clustering experiment if the size of the set of all-clusters increases then the size of the minimum size for the clusters to be considered statistically significant increases too.

We believe that the reason for the decrease in the number of significant clusters is that the minimum size for significant clusters also increased. Therefore, although MAXCCLUS found more clusters, fewer of them were 
counted as significant, and therefore, it was not able to explain as much of the data.

\section{Instances}

On average, after adding descriptors, there is a decrease in the number of instances that MAXCCLUS can describe (figure 6.8). The instances described are almost as the same as the ones described before adding descriptors: a high percentage of the instances are retained (figure 6.9), and a low percentage of the instances are new (figure 6.10). Therefore after adding descriptors MAXCCLUS is not describing more instances than before adding descriptors, instead it is describing fewer instances on average and these instances are almost the same instances that MAXCCLUS was able to describe without the added descriptors.

Having a different set of clusters describing almost the same set of instances means that adding descriptors cause MAXCCLUS to spend more time in clustering (figure 6.18) without being able to increase the number of instances that it is able to describe.

\section{Rules}

After adding descriptors, MAXCCLUS creates many more rules than before adding words, to characterise the clusters it found (figure 6.11). Note that these rules characterise almost the same set of instances as before. Having many more rules is confusing and does not help a user trying to make sense of the clustering results. The number of rules increased dramatically because MAXCCLUS can create many alternative rules using the added words that have the similar meaning. 
In some cases, the number of rules for a cluster does not change, but even then, the rules may be different. For example, figure 6.19 shows a significant cluster that MAXCCLUS creates when clustering before adding descriptors. This cluster has 278 instances that are described by the rule "protein, region". Using the same data set, when clustering after adding synonyms, MAXCCLUS creates the same cluster that has the same 278 genes, but with different rule, "protein, neighborhood", to describe the instances (figure 6.20).

What happened is that the previous version of the cluster has the words "protein" and "region" in the set of the Necessary words. After adding synonyms, the descriptors "area", "domain", "neighborhood", and "part" were added to all the instances that had the word "region" in their descriptor set. These synonyms were added because they exist in the set of all-descriptors (other synonyms of the word "region" and the synonyms of the word "protein" were not added because they do not exist in the set of all-descriptors). When clustering after adding synonyms, the word "neighborhood" replaced the word "region" in the set of the Necessary words, therefore the rule that describe the instances became "protein, neighborhood" (figure 6.20).

This seems ok but when comparing the frequency of the word "region" before adding descriptors, which is 2002, with the frequency of the word "neighborhood" which is 1 , the word "region" is more familiar than the word "neighborhood". Before adding descriptors, the word "region" appears in the descriptor sets of 2002 instances, in contrast the word "neighborhood" appears only in the descriptor set of 1 instance. Which make the word "region" more frequent in use than the word "neighborhood". It is not desirable to have a much less frequent word replacing a more frequent one in a rule, because the more frequent word is probably more familiar than its synonyms to the people that work in the domain (in this case the genetics scientists), and may have important connections with the domain. 
Cluster (Up, 95\% accuracy, 278 instances)

Characterisation:

Necessary: protein, region

Rule: protein, region

Figure 6.19: A cluster before adding synonyms

Cluster (Up, 95\% accuracy, 278 instances)

Characterisation:

Necessary: $\quad$ protein, neighborhood

Supplementary: region, part, area, domain

Rule: protein, neighborhood

Figure 6.20: The same cluster of figure A.1 but having different rule after adding synonyms.

On the other hand, in most of the cases the number of rules increases after adding descriptors. For example suppose that, when clustering before adding descriptors, MAXCCLUS finds a rule that has the following words: w1, w2, and w3. Suppose that $s 1$ is a synonym for $w 1, s 2$ is a synonym for $w 2$, and s3 is a synonym for w3. Therefore, when clustering after adding using SY, to characterise a cluster MAXCCLUS creates the rule "w1, w2, w3" and its alternatives, which are:

"s1, w2, w3"

"w1, s2, w3"

"w1, w2, s3"

"s1, s2, w3"

"s1, w2, s3"

"w1, s2, s3"

"s1, s2, s3" 
to characterise the same cluster. These alternative rules are likely to confuse the user who is trying to make sense of the cluster. Therefore, adding descriptors using SY is not useful. The same process happens when clustering using $\mathrm{HY}$, but the increase in the number of rules, although it is not as great as when using SY, is still a large increase. Suppose that h1 is a hypernym for the $w 1, h 2$ is a hypernym for the w2, and h3 is a hypernym for the w3.

The worst case is when using $\mathrm{SH}$ where the number of rules jumps very high for the significant clusters. This is because using $\mathrm{SH}$ is combining the use of the approaches SY and HY. Therefore, the number of rules when using $\mathrm{SH}$ worsens the rules' results, even more than when using SY or HY, by dramatically increasing the number of rules giving the worst results. Although it is still high, the number of rules of the cover cluster set is not as high as that of the significant cluster set, therefore covering the significant clusters with the cover cluster set is working fine. Even more, the generalisation is working fine because the number of rules of the general cluster set, although it is still a large increase, is the best case among the three sets of clusters, which reflects an enhancement of the generalised cover cluster set over the cover cluster set. 


\subsection{Conclusion and recommendations}

Adding synonyms, hypernyms (or both) to the descriptor sets of the instances does not help MAXCCLUS to describe more instances, instead MAXCCLUS describes fewer instances, takes more time for clustering, and produces clusters that are characterised with many alternative rules that can confuse the user who is trying to make sense of the clusters. Therefore adding synonyms, hypernyms or both of them is a bad idea. It may be possible to get better clustering results if we can add synonyms and/or hypernyms of the domain-relevant words but WordNet does not contain all of these. It might be better to replace the descriptors with their most frequent (relevant to the textual data) synonyms or hypernyms; this will prevent MAXCCLUS from creating alternative rules.

A topic for future work is to replace the domain-relevant descriptors with there frequent synonyms and/or hypernyms. 


\section{Chapter 7}

\section{Five Categories}

This chapter describes a clustering experiment designed to study the ability of MAXCCLUS to cluster instances that have five adjacent categories and comparing its results with the reference experiment where MAXCCLUS clusters instances that have two distinct categories only. Unfortunately, the result of this chapter is to show that MAXCCLUS is not as good for clustering instances that fall in multiple adjacent categories, as it is for clustering instances that belong to two clearly distinct categories.

This clustering experiment uses the same data that the reference clustering experiment uses, but with an important difference: the number of the categories and how close the categories are. The reference clustering experiment uses two categories to categorise instances (genes). The two categories are Up and Down. On the other hand, this chapter's clustering experiment uses five categories: Up, MidUp, Mid, MidDown, and Down. Of these five categories, the $U p$ and Down categories are the same $U p$ and Down categories as in the reference clustering experiment.

The actual raw experimental data allocates each gene a numeric expression ratio, which varies between approximately 0.001 and 1000.0. The discrete categories represent subranges of the expression ratio. In both the two categories and the five categories experiments, the Up and Down represent the ranges: greater than or equal to 2.0 , and less than or equal to 0.5 respectively (Figure 7.1). In the five categories experiment, the categories MidUp, Mid, and MidDown cover the remaining part of the range. It is significant that in the two categories experiment, all the genes in the range between Up and Down are excluded, so that the two categories are quite 
distinct. In the five categories experiment, all the genes are included, so that the categories are adjacent to each other and are less distinct. Also, the five categories experiment includes 3,548 genes, whereas, on average, only 921 genes are included in each data set of the two categories experiment.

\begin{tabular}{|l|l|}
\hline Category & Expression ratio $\mathrm{X}$ \\
\hline Up & $2.0 \leq \mathrm{X}$ \\
\hline MidUp & $1.4<\mathrm{X}<2.0$ \\
\hline Mid & $0.7 \leq \mathrm{X} \leq 1.4$ \\
\hline MidDown & $0.5<\mathrm{X}<0.7$ \\
\hline Down & $\mathrm{X} \leq 0.5$ \\
\hline
\end{tabular}

Figure 7.1: Categories versus their expression ratios.

\subsection{Textual data}

The textual data, for the five categories clustering experiment is the same as that of the reference clustering experiment; it represents the association of each gene's id with the gene's descriptors.

\subsection{Experimental results}

The results shown in the figures below are for running MAXCCLUS on 19 of the microarray experimental data sets (data set: $3,6,7,10,11,12,13,16,18$, $20,21,22,23,24,25,27,28,29$, and 30 ), using five categories instead of two categories. Figures 7.2 to 7.13 show the results for the number of clusters, the number of instances, the number of rules, the number of words in the rules, and the number of words per rule. 


\section{Chapter 7: Five Categories}

\section{The clusters}

Figure 7.2 shows the changes to the total number of clusters when MAXCCLUS clusters instances using five categories instead of two categories.

On average, there are fewer clusters when clustering instances using five categories, than when clustering instances using two categories (between $18 \%$ and $47 \%$ as many). The significant cluster set has greater decrease in the number of clusters than the cover cluster set, which in turn has greater decrease than the generalised cover cluster set.

The distributions show that the significant cluster set has the narrowest distribution (the maximum value is an outlier) of the cluster sets, the narrowest inter-quartile range, it has the lowest median, and is skewed to lower values. The cover cluster set is more spread in its distribution, has a higher median but is still skewed to the lower values. The generalised cover is has the most spread distribution and the highest median.

When clustering instances using five categories a completely different set of clusters was found: no clusters were retained from the clusters found when clustering instances using two categories, and therefore all the clusters are different from the clusters found when clustering instances using two categories.

No clusters found from clustering instances using two categories are subsumed by new clusters found from clustering instances using five categories. 


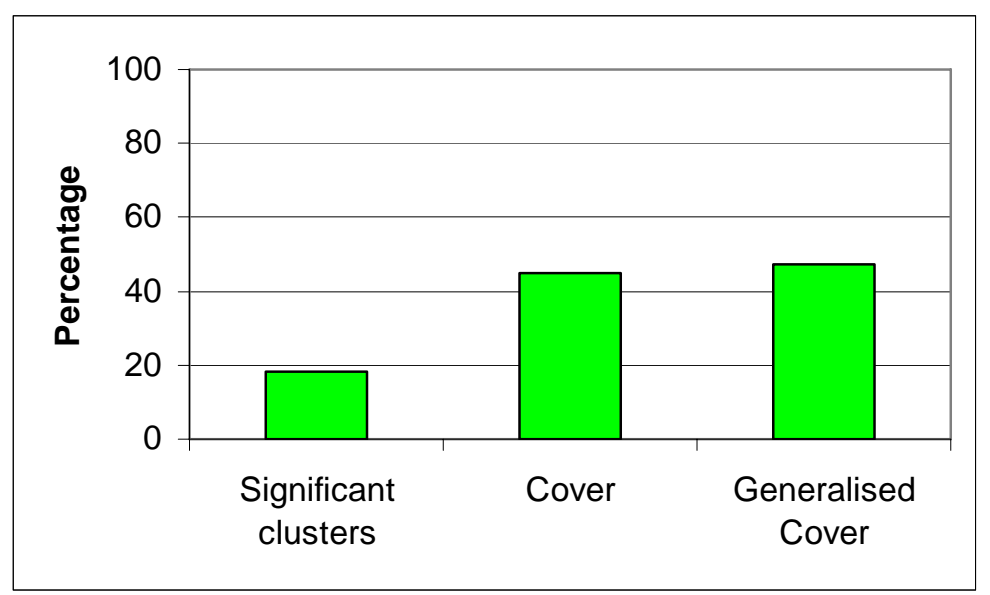

a: Average

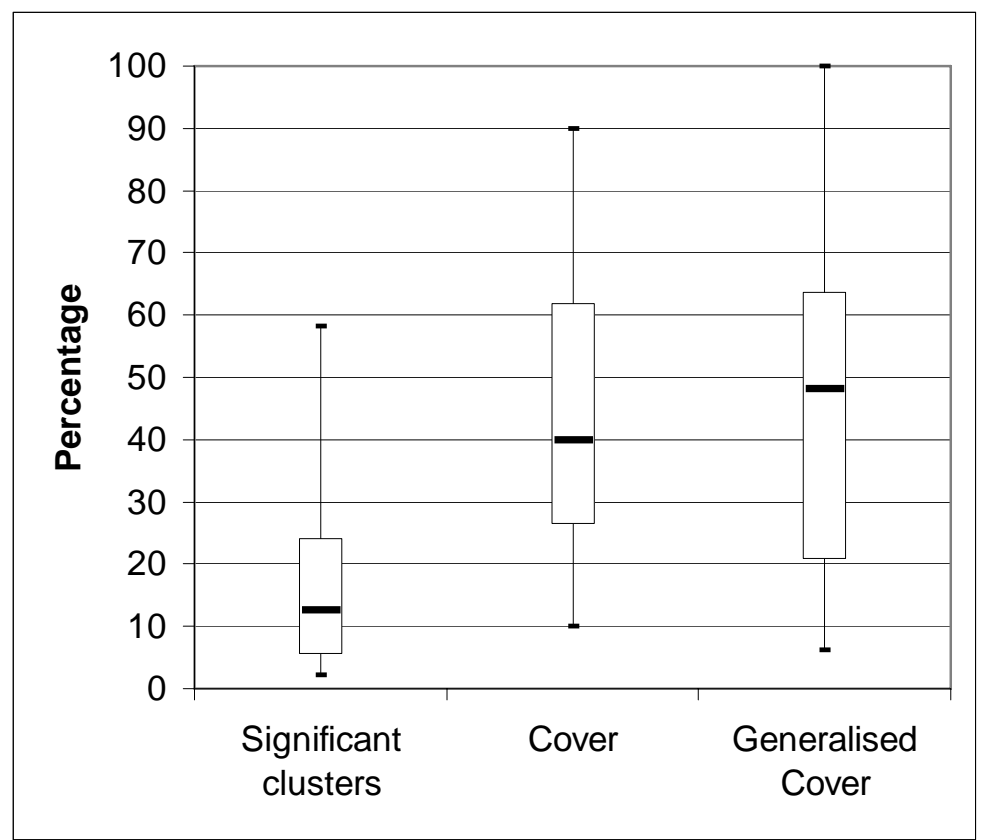

\section{b: Distributions}

Figure 7.2: Change to the total number of clusters

\section{Significant clusters minimum size}

Figure 7.3 shows the changes to the minimum size of the significant clusters for the Up and Down categories when clustering using five categories compared with when clustering using two categories. Only the minimum size for the Up and Down can be compared because there are no other categories available when clustering genes using two categories. 
When clustering instances using five categories, on average, the significant clusters' minimum sizes for the Up and Down categories are less than that when clustering instances using two categories.

On average, the significant clusters' minimum size for the Up category has greater decrease than that for the Down category.

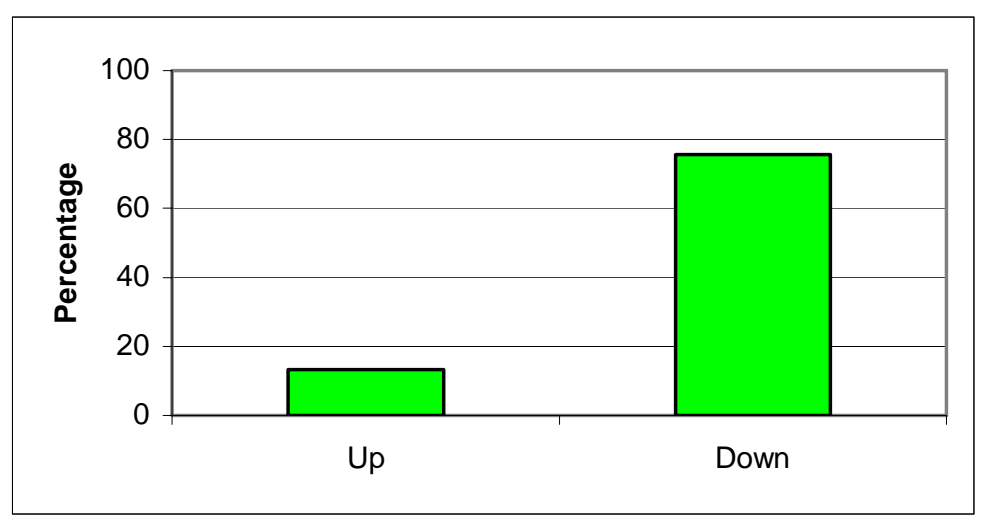

Figure 7.3: Average change to the significant clusters' minimum size for the Up and Down categories

On the other hand, Figure 7.4 shows the average of the significant clusters' minimum size for each of the five categories (not as a percentage value). The figure shows that the Mid category has higher minimum significant cluster size than the other categories.

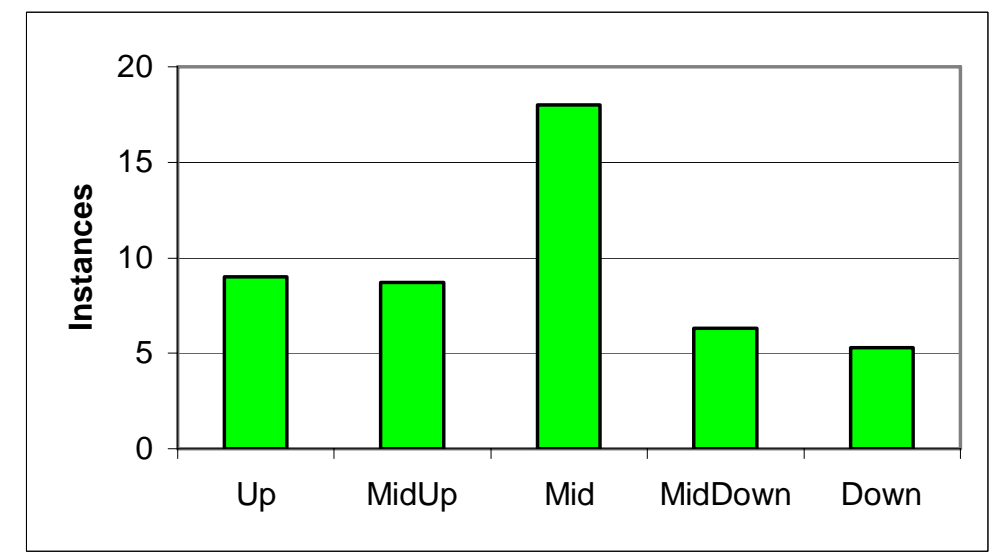

Figure 7.4: Average of the significant clusters' minimum size for each of the five categories (not as a percentage value) 


\section{The instances}

Figures 7.5 to 7.7 show the changes to the number of instances when MAXCCLUS clusters instances using five categories instead of two categories. The figures show the changes to the total number of instances, the number of retained instances, and the number of new instances.

In each figure, the results of the significant and the cover cluster sets are the same because both sets should cover the same instances.

On average, there are fewer instances when clustering instances using five categories, than when clustering instances using two categories (about 20\% as many) (figure 7.5), in spite of the fact that there are on average of four times as many instances in the experimental data sets.

Although the averages for all the cluster sets are the same, the distributions in show that the significant and the cover cluster sets have a narrower interquartile range and are more skewed towards the minimum values with slightly lower median than the generalised cluster set (figure 7.5). All the maximum values are outliers and they belong to one data set (data set 13), while the value next to the maximum value is about $45 \%$, which is not an outlier. Also, all the minimum values belong to another single data set (data set 20 ).

When clustering instances using five categories, on average, very few instances are retained from the clusters found when clustering instances using two categories (about 6\%) (Figure 7.6), and about 56\% of the instances are different from the instances found when clustering instances using two categories (Figure 7.7).

Although Figure 7.7 shows that the average of the number of new instances is almost the same for all the cluster sets, the distribution show that the median of the generalised cover cluster set is lower than that of the other cluster sets. Also this figure shows that all the cluster sets have a very widely spread distribution; all have a wide inter-quartile range especially the generalised 
cover. Three data sets (data set 3,13 , and 23 ) share all the maximum values, while two data sets (data sets 6 and 7) share all the minimum values.

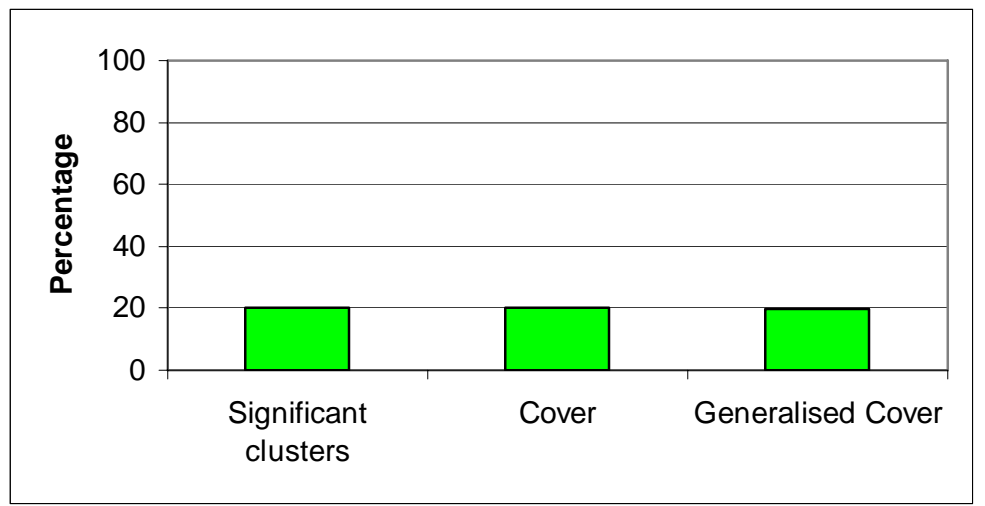

a: Average

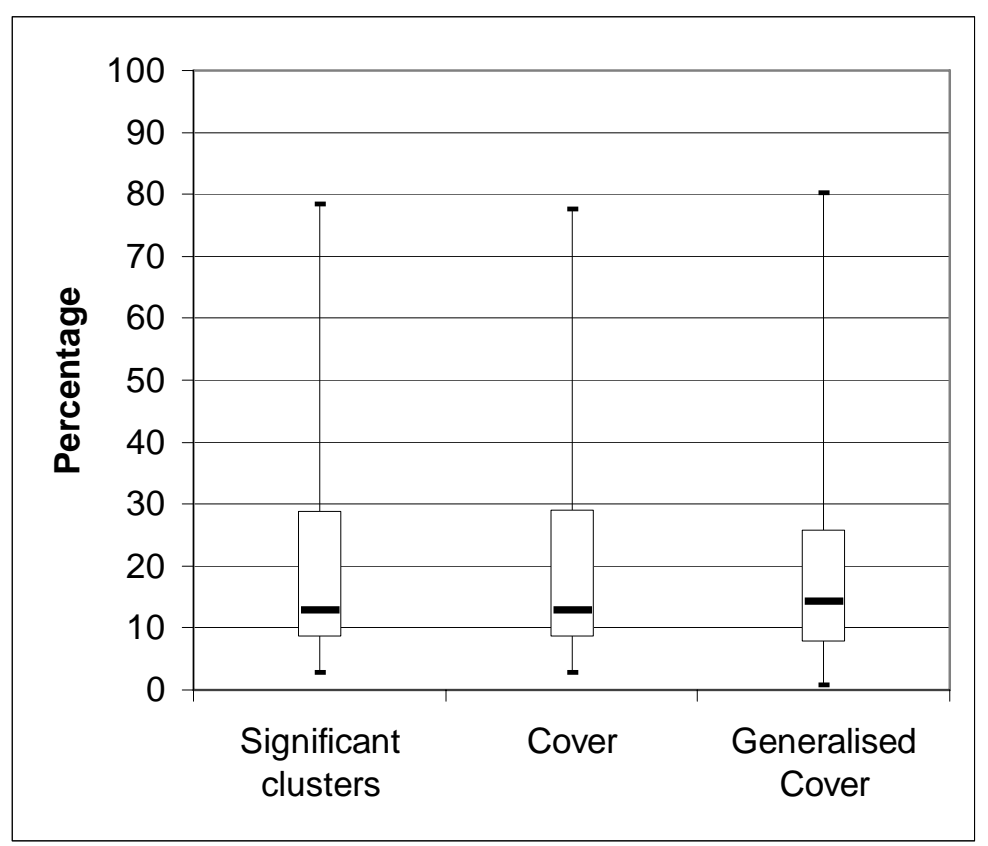

b: Distributions

Figure 7.5: Change to the total number of instances 
Chapter 7: Five Categories

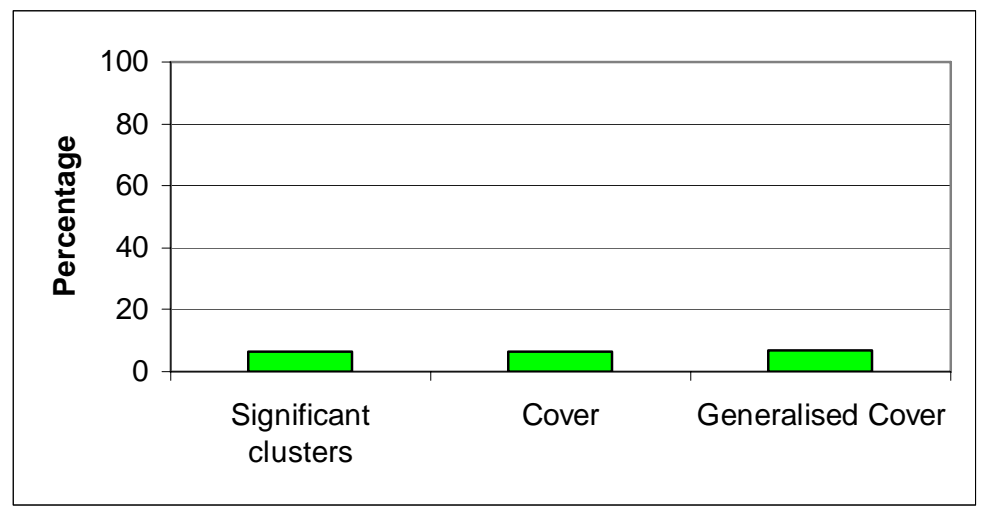

Figure 7.6: Average number of retained instances

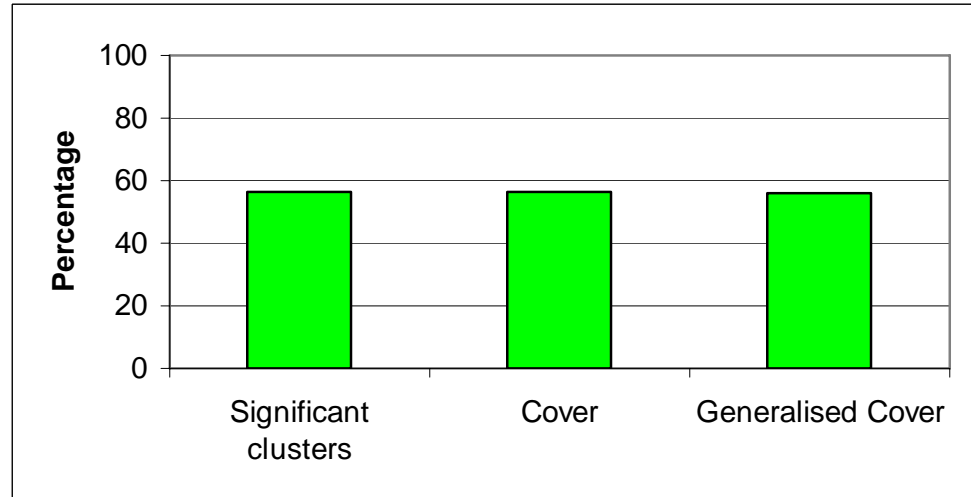

\section{a: Average}

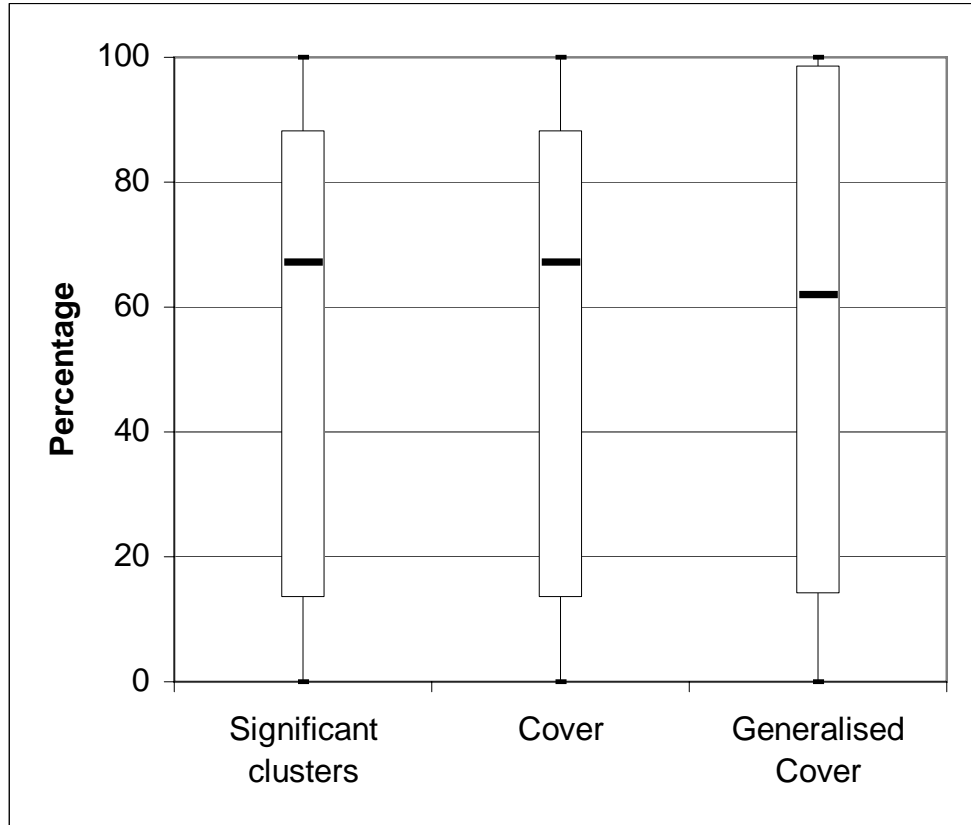

b: Distributions

Figure 7.7: Number of new instances 
Figure 7.8 shows the average of the number of instances per category for each of the five categories when clustering using five categories (not as a percentage value). The figure shows that the Mid category has the highest average number of instances per category.

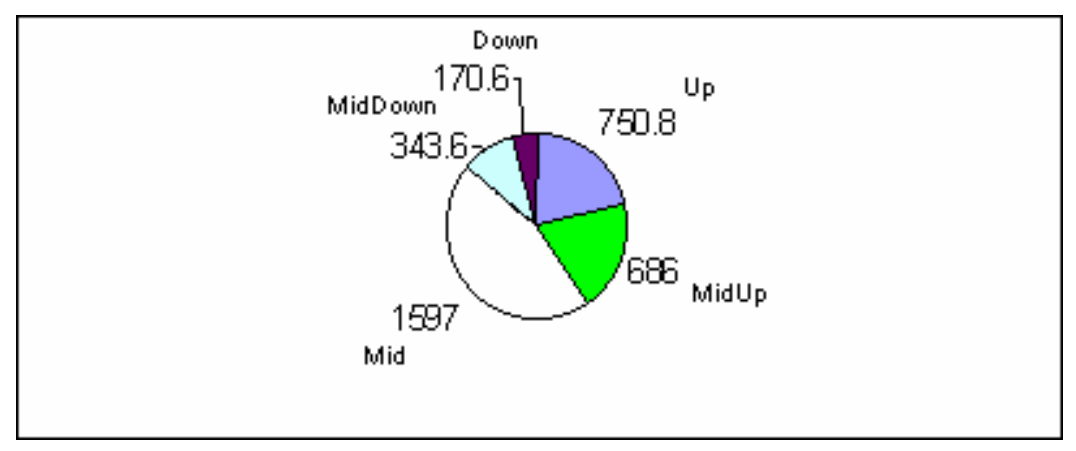

Figure 7.8: Average of the number of instances per category for each of the five categories when clustering using five categories (not as a percentage value)

\section{The rules}

Figure 7.9 shows the changes to the total number of rules when clustering instances using five categories instead of two categories.

On average, there are fewer rules when clustering using five categories, than when clustering using two categories (between $34 \%$ and $76 \%$ as many). The significant cluster set has greater decrease in the number of rules than the cover cluster set, which in turn has greater decrease than the generalised cover cluster set.

When clustering instances using five categories, no rules are retained from the rules found when clustering instances using two categories, and no rules found from clustering using two categories are subsumed by new rules found from clustering using five categories. 
The distributions show that the significant clusters have a narrower distribution than the other cluster sets. The maximum values of the cover and the generalised cover cluster sets belong to data set 12 , while the minimum values of the significant and the cover cluster sets belong to data set 24 .

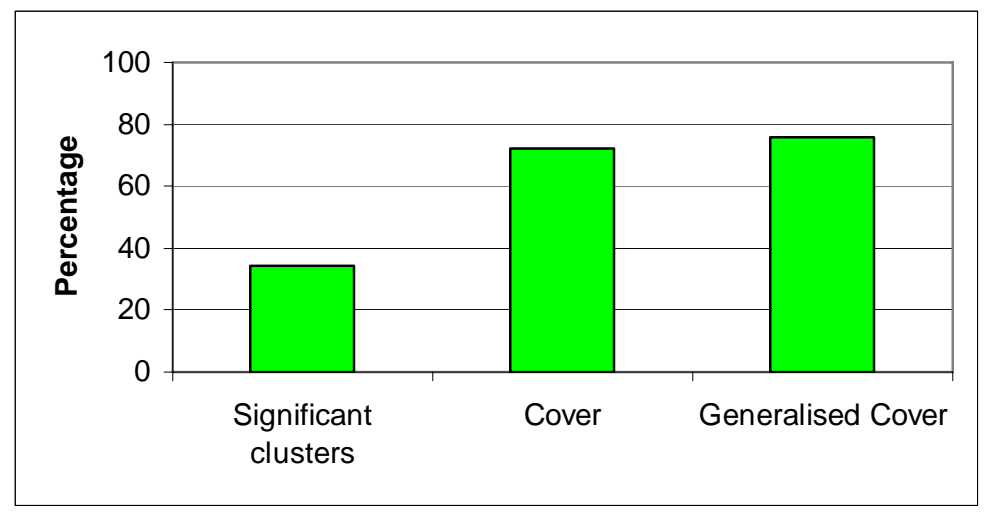

a: Average

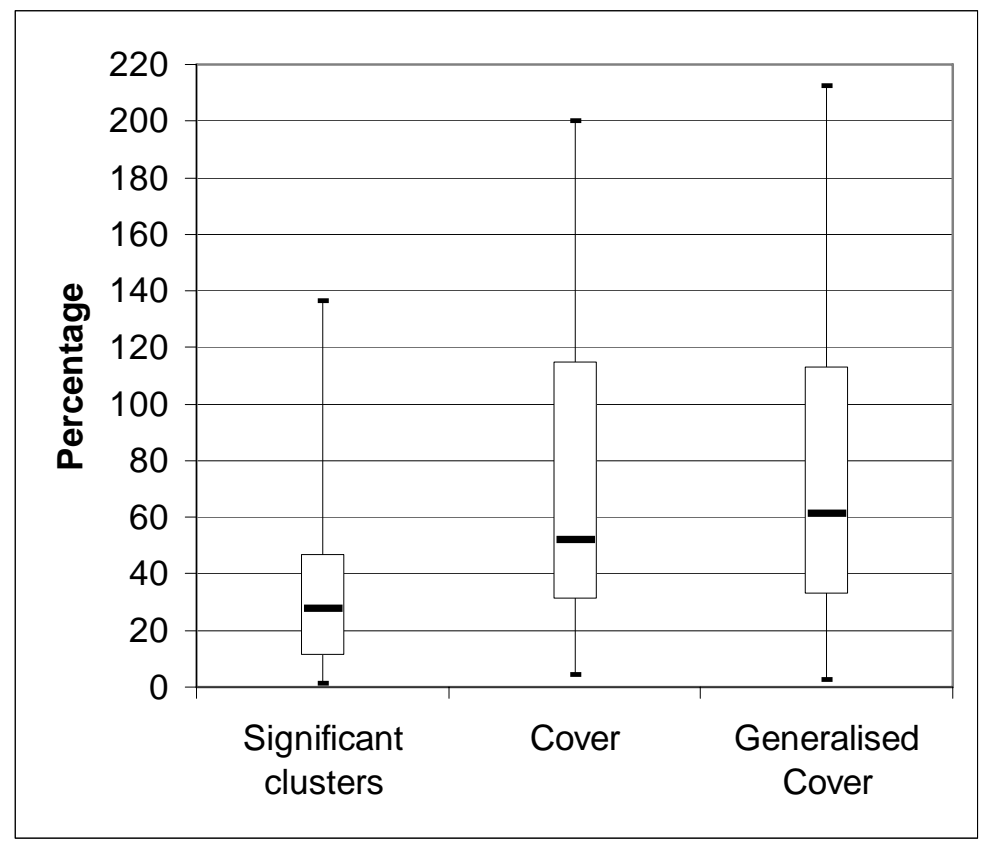

b: Distributions

Figure 7.9: Average change to the total number of rules 


\section{The rules' words}

Figures 7.10 to 7.12 show the changes to the number of rules' words when clustering using five categories instead of two categories. The figures show the changes to the total number of rules' words, the number of retained rules' words, and the number of new rules' words.

On average, there are fewer rules' words when clustering using five categories, than when clustering using two categories (between 57\% and $74 \%$ as many). The significant cluster set has greater decrease in the number of rules' words than the cover and the generalised cover cluster set (figure 7.10). The distributions show that there is a wide variation across the data sets.

When clustering using five categories, on average, between 33\% and $35.4 \%$ of the rules' words are retained from the rules found when clustering using two categories (figure 7.11). The average is almost the same for all the cluster sets.

When clustering using five categories, on average, between $42 \%$ and $53.5 \%$ of the rules' words are different compared with the rules' words found when clustering using two categories (figure 7.12). The significant cluster set has fewer different rules' words than the other cluster sets. 


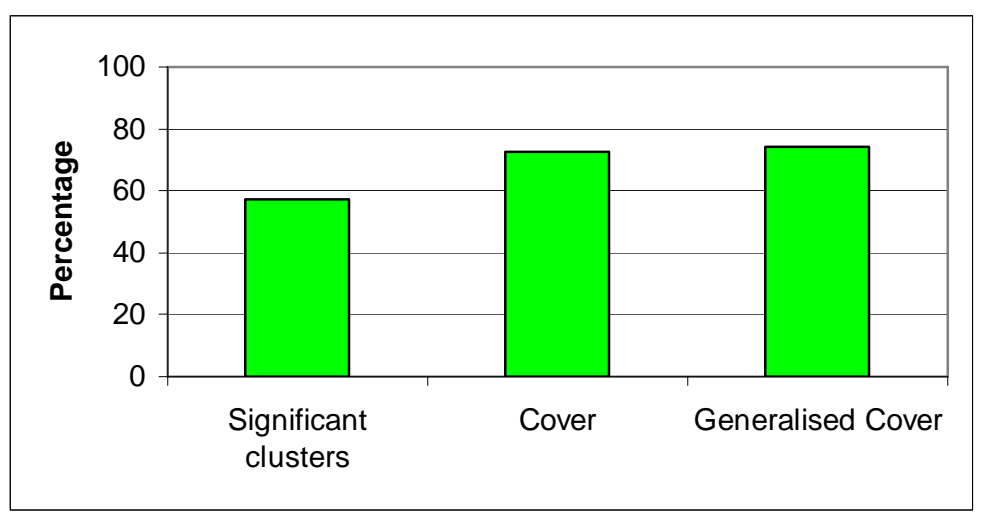

a: Average

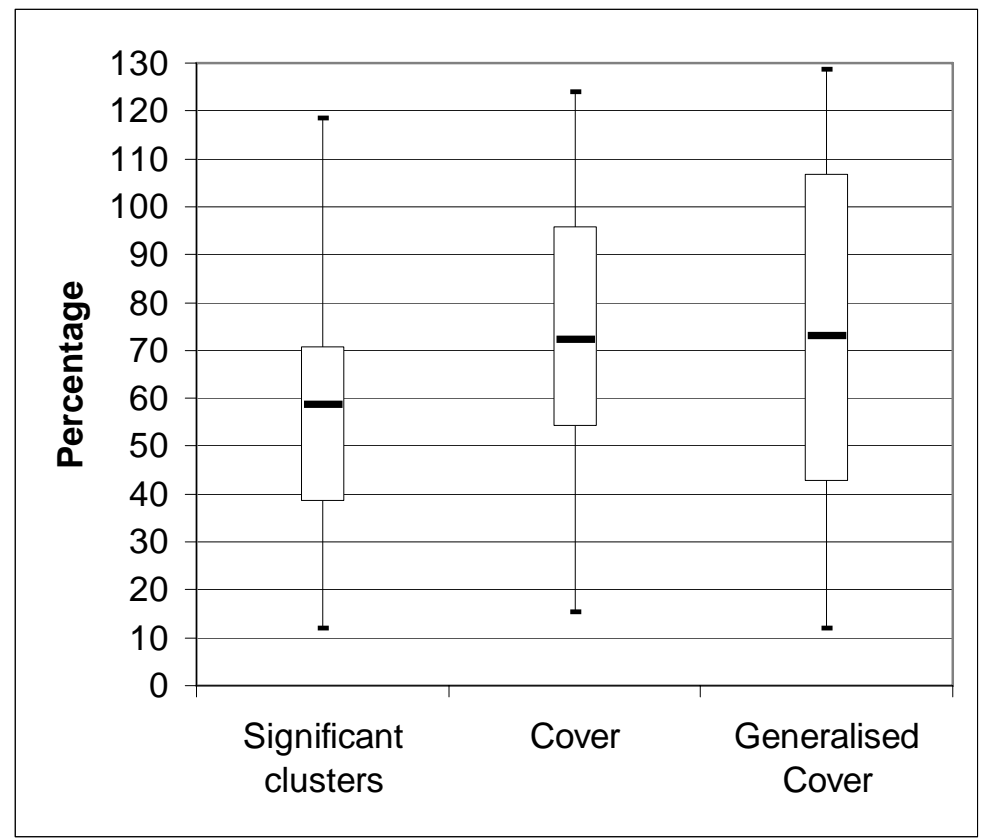

\section{b: Distributions}

Figure 7.10: Change to the total number of rules' words

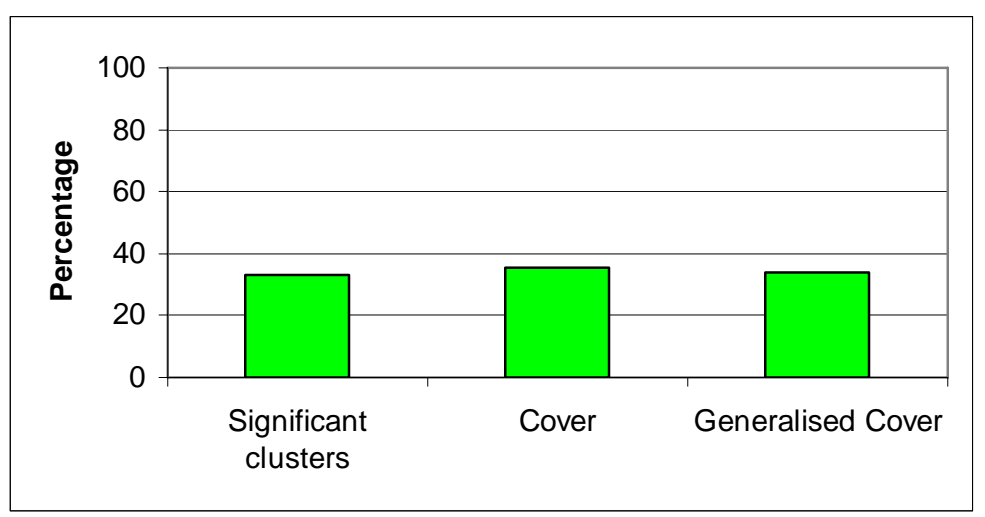

Figure 7.11: Average number of retained rules' words 


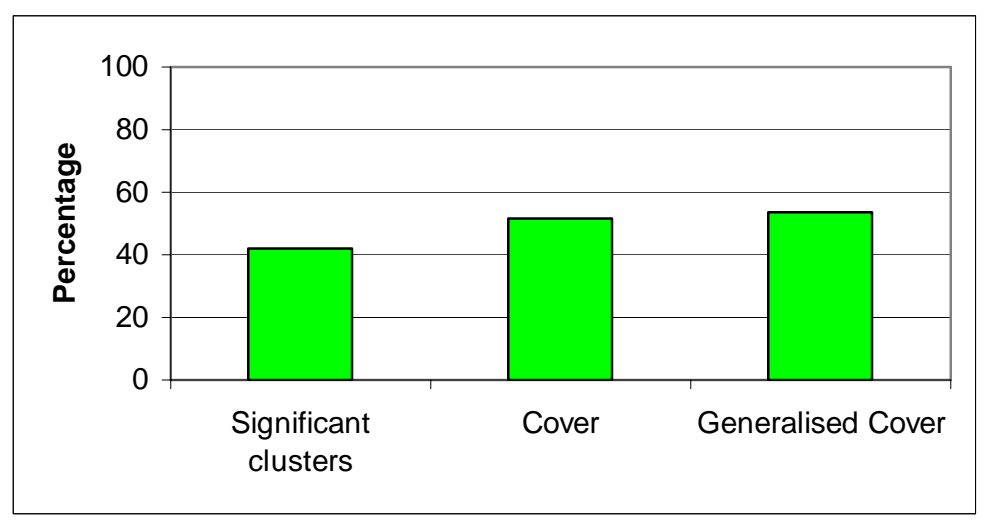

Figure 7.12: Average number of new rules' words

\section{The words per rule}

Figure 7.13 shows the changes to the number of words per rule when clustering using five categories instead of using two categories

On average, there are more words per rule when clustering using five categories, than when clustering using two categories (between 136\% and $151 \%$ as many). The generalised cover cluster set has a greater increase in the number of words per rule than the other cluster sets.

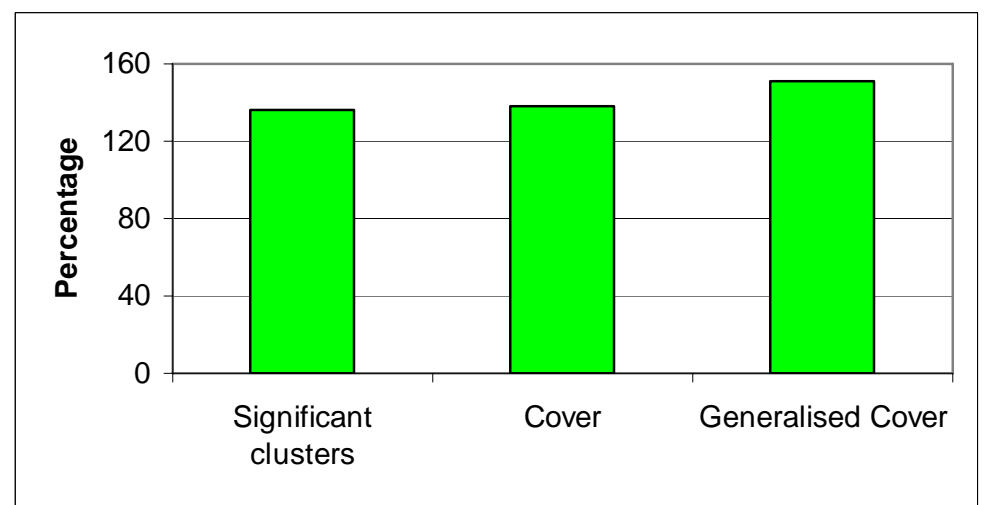

Figure 7.13: Average change to the number of words per rule 


\section{The execution time}

When clustering using five categories, on average, the execution time is much more than that when clustering using two categories - about 15 times longer when using five categories compared with the execution time when clustering using two categories.

\subsection{Analysis and discussion}

The following sections analyse and discuss the results shown in the experimental results section.

\section{Clusters}

On average, there are fewer clusters when clustering instances using five categories, than when clustering instances using two categories, especially the significant cluster set (figure 7.2). The clusters MAXCCLUS creates when clustering instances using five categories are completely different from the clusters it creates when clustering instances using two categories.

The difference in the clusters is the result of including many new instances (the ones with categories MidUp, Mid or MidDown) in the data sets in addition to the ones used in the reference clustering experiment (the ones with categories Up or Down).

When clustering instances using five categories, the minimum size of clusters to be considered statistically significant clusters by MAXCCLUS, decreases for the Up and Down categorised clusters compared to that for clustering instances using two categories (figure 7.3). The minimum size of the Down categorised clusters decreased from about 7 instances to about 5 instances, 
and the minimum size of the Up categorised clusters decreased from about 89 instances to about 9 instances.

The reason for the reduction in the minimum size for the Up and Down clusters is not because the size of the set of all-clusters decreased, since that increased to 450,000 clusters for all the data sets when clustering instances using five categories (it is on average only 35,000 clusters, when clustering instances using two categories). The set of all-clusters is the set of clusters that MAXCCLUS creates from the descriptor sets of the data set's instances. Usually for a clustering experiment if the size of the set of all-clusters increases then the size of the minimum size for the clusters to be considered statistically significant clusters increases too.

When MAXCCLUS clusters instances using only two possible categories (Up and Down) MAXCCLUS would have to have large clusters to be considered significant, because there is a good chance that most of some cluster's instances belong to one category when there are only two possibilities. On the other hand when MAXCCLUS clusters instances using five possible categories, a small pure cluster would be considered significant because it is very unlikely that, by chance, most of any cluster's instances belong to a single category when there are five possibilities.

Notice that, on average, the minimum size of a cluster to be considered statistically significant (figure 7.4), increases with the number of instances for this category in the data set (figure 7.8). The minimum size of the Mid categories clusters is more than that of any other category clusters, because the number of the instances categorised as Mid regulated instances is more than the number of any other category instances.

Although the minimum size of a cluster to be considered significant reduces when clustering using five categories, the number of significant pure clusters unexpectedly decreases considerably instead of increasing (figure 7.2). The reduction in the number of significant pure clusters means it is harder to find significant clusters that are pure in the real data when clustering using five 
categories than when clustering using two categories. To explain this, we give an example.

Suppose, when clustering using two categories, there is an Up cluster of size 7 , and the purity is required by the user to be $100 \%$, which means that a cluster can be pure only if all its instances have the same category; in other words MAXCCLUS excludes from the significant pure cluster set any Up cluster that has at least one instance not of Up category. On the other hand when clustering using five categories two more instances that have some words in common with the cluster were added to it to become a cluster of size 9. These two new instances are MidUp regulated. MAXCCLUS excludes this cluster from the significant pure cluster set even though the MidUp instances (which are not clearly Up regulated) could be in reality Up ones but their regulations were measured as MidUp because of microarray experimental noise. Therefore, the cluster becomes bigger but also becomes impure.

When clustering using two categories MAXCCLUS can easily create rules that distinguish between the two separated categories (Up and Down). But when using five categories MAXCCLUS has difficulty creating rules that can distinguish between any two adjacent categories that do not have a gap in between (Up and MidUp, MidUp and Mid, Mid and MidDown, or MidDown and Down). This is not the behaviour of MAXCCLUS only. In [Sahami, M; Dumais, S; Heckerman, D; and Horvitz, E. 1998] they mentioned the same behaviour with their Spam filtering system, where the system was able to distinguish between ordinary and Spam messages, but it behaved poorly when it attempts to distinguish between three kind of messages: ordinary messages, one kind of Spam messages and other kind of Spam messages. 


\section{Instances}

On average, when clustering instances using five categories, there is a large decrease in the number of instances that can be described - only $20 \%$, even though this includes the instances that have the categories MidUp, Mid, or MidDown which cannot be described when clustering using two categories (figure 7.5). This decrease in the number of described instances is despite the fact that all the 3548 instances, available from the microarray raw experimental data, are included in all the experimental data sets that MAXCCLUS used to cluster using five categories.

Furthermore, the individual instances that MAXCCLUS is able to describe when clustering using five categories are quite different from the instances it can describe when clustering using two categories: a high percentage of the instances are new, about 56\% (figure 7.7). The primary reason is the reduction in the number of significant pure clusters (as explained in the previous section), since only instances in significant pure clusters are explained.

The algorithm does not scale well to several thousands of instances; the cost of the search grows too fast. The time was much longer because of more instances for the data sets and more descriptors contributed by them. Note that it is the significance test, rather than the construction of the clusters that dominates the time.

\section{Rules and Words per rule}

On average, there are fewer rules when clustering instances using five categories, than when clustering instances using two categories, especially the significant cluster set (figure 7.9), and all the rules are different. The number of rules does not decrease as much as the number of clusters (figure 7.2), nor as much as the number of instances (figure 7.5). This means that each cluster must have more possible rules because there are many 
alternative rules due to adding new instances that contribute with their descriptors.

This is a consequence of having smaller clusters, which therefore are likely to have more words in common. If there are many words associated with the cluster, there are likely to be many possible combinations of these words (that is many rules) that will distinguish just the instances in the cluster.

The words per rule increased (figure 7.13) because of adding new instances (the ones that have categories MidUp, Mid, or MidDown) to the data sets for clustering using five categories. Because there are more instances altogether, MAXCCLUS needs to use more words in each rule to be able to exclude the instances that do not belong to the cluster that the rule characterise (describe).

\section{Rules' words}

In spite of more words per rule, there are still fewer words altogether in the rules when clustering instances using five categories, than when clustering instances using two categories, especially the significant cluster set (figure 7.10). The rules' words when clustering instances using five categories are quite different from the rules' words when clustering instances using two categories: on average, only about $34 \%$ of the rules' words are retained (figure 7.11 ), and between $42 \%$ and $54 \%$ of the rules' words are new (figure 7.12).

The difference in the rules' words is because of the difference in the rules that previous section describes. 


\subsection{Conclusion}

MAXCCLUS is not able to produce as good results when clustering using continues range of categories, as when clustering using two categories separated with a clear gap. It is not able to explain nearly as many instances, even though it constructs almost as many rules as when clustering using two categories. When using continues range of categories (Up, MidUp, Mid, MidDown, and Down) it is difficult for MAXCCLUS to distinguish between any two adjacent categories that do not have a gap in between (Up and MidUp, MidUp and Mid, Mid and MidDown, or MidDown and Down). With respect to the clustering time, the algorithm does not scale well to several thousands of instances as the cost of the search grows too fast.

\subsection{Recommendations}

If one needs to use MAXCCLUS to cluster instances that fall in more than the clearly Up or clearly Down categories, it is better to be sure that the categories are separated with a gap. Therefore one might be able to use MAXCCLUS to cluster instances that fall in three categories Up, Mid, and Down, where there is a gap between Up and, Mid, and between Mid and Down (figure 7.14). Or clustering by using two categories separated by a gap, one category is Up-MidUp (by merging Up and MidUp), and the other is Down-MidDown (by merging Down and MidDown), in this case the gap would be ignoring the instances that belong to the Mid category (figure 7.15). These two suggested clustering experiments are for future research. 


\section{Chapter 7: Five Categories}

\begin{tabular}{|l|l|}
\hline Category & Expression ratio $\mathbf{X}$ \\
\hline Up & $2.0 \leq \mathrm{X}$ \\
\hline Mid & $0.7 \leq \mathrm{X} \leq 1.4$ \\
\hline Down & $\mathrm{X} \leq 0.5$ \\
\hline
\end{tabular}

Figure 7.14: Recommended three categories versus their expression rations with gaps in between

\begin{tabular}{|l|l|}
\hline \multicolumn{1}{|c|}{ Category } & Expression ratio $\mathbf{X}$ \\
\hline Up-MidUp & $1.4<\mathrm{X}$ \\
\hline Down-MidDown & $\mathrm{X}<0.7$ \\
\hline
\end{tabular}

Figure 7.15: Recommended two categories versus their expression ration separated with a gap (it is different version than that we used in this project) 


\section{Chapter 8 Tools}

The focus of the project was on the experiments that investigated various modifications to MAXCCLUS and its data. However, the project also required the development of several tools to assist in the conduct of these experiments. This chapter briefly describes these tools.

\subsection{Modifications to MAXCCLUS and its data}

We modified MAXCCLUS by designing and implementing the exhaustive search generalisation (used in chapter 3) using the Java computer programming language. We modified the data that MAXCCLUS used using the Perl computer programming language.

\subsection{Comparison used in methodology}

To carry out the task of comparison of thousands of the objects in tens of XML files that MAXCCLUS produced as the results of its clustering throughout all the clustering experiments, we designed and implemented computer programs using the Java computer language with SAX (Simple API for XML) and Xerces parser from Apache, to parse the XML files, extract the knowledge, compare, and report the comparison results. 


\subsection{Rendering}

MAXCCLUS reports its clustering results for a microarray experiment data set as an XML file. To easily view these results, they need to be rendered to HTML (HyperText Markup Language) files. Rendering was originaly done by running a Java program on each of the XML files to create the respective HTML file. This approach was not flexible enough in case one needs to change how the HTML presents the data; furthermore this approach doubles the computer storage size needed to store the clustering results (because of having both the XML and the HTML files of the results), which can be considerable when running MAXCCLUS on many data sets (some XML and HTML files can exceed 34MB in size). We modified the rendering by using only one small (7KB) XSL (Extensible Stylesheet Language) file to render, on the fly, any XML file created by MAXCCLUS, to a HTML file by using Microsoft Internet Explorer to open the XML file.

\subsection{Web robot}

Some microarray experiment data sets have gene ids that are not in the same format of the Swiss-Prot textual database. To be able to use these gene ids, we need to map these ids to the format that Swiss-Prot uses. This can be done by accessing websites of some gene databases and submitting a query to get back a web page that contains different gene id formats from which we can select the id that has the Swiss-Prot format.

Doing this manually is not a problem for few genes, but for thousands of genes it is time consuming, error prone, and frustrating. Therefore we designed and implemented a web robot, using the Perl computer programming language, to carryout the task automatically. 
To retrieve the Swiss-Prot format gene ids of the non Swiss-Prot format gene ids, we chose the web site of the $\mathrm{NBCl}$ (National Center for Biotechnology Information) at "http://www.ncbi.nih.gov", which is a resource for molecular biology information and public databases.

In one run of the web robot program, the web robot access the web site of the $\mathrm{NBCl}$ thousands times (depending on the number of genes). In each access, it submits a query for one gene id (saved previously in a file of gene ids), waits for the web page that holds the results of the query, extracts the SwissProt gene id format from the results page, and save the gene id with its SwissProt id format into a gene ids mapping file. We can then use the gene ids mapping file when we extract the textual data from the Swiss-Prot database.

\subsection{Clusters visualisation}

We needed a way to visualise the clusters to get some idea about how the instances are distributed among the clusters of the results of MAXCCLUS clustering for a microarray experiment data set.

We designed and implemented a program using the Java computer programming language to automatically produce PNG (Portable Network Graphics) image files from the $X M L$ (Extensible Markup Language) clustering result files that MAXCCLUS produce. We chose the PNG format for the images because it is patent-free unlike, some other image formats.

The images produced are usually big. Figure 8.1 shows one of the relatively small images that it can be viewed clearly on an A4 size page. On the other hand Figure 8.2 shows another image, which is not clearly viewable on an A4 size page because the image was shrunk to fit the page size, but it can still give some idea about the clusters and their instances (if the reader is viewing 
a PDF - Portable Document Format - file of this thesis, the image can be viewed clearly by magnifying the document).

In the images, the $x$ and $y$ coordinates represent the clusters and the instances respectively. The clusters are sorted from left to right in ascending order with respect to the number of instances they have. The instances are sorted from bottom to top in an ascending order with respect to the number of clusters they are in.

The vertical coloured lines are for the different categories of the clusters. The horizontal coloured boxes represents the instances, we made them coloured to make it easy to distinguish between the adjacent instances. 


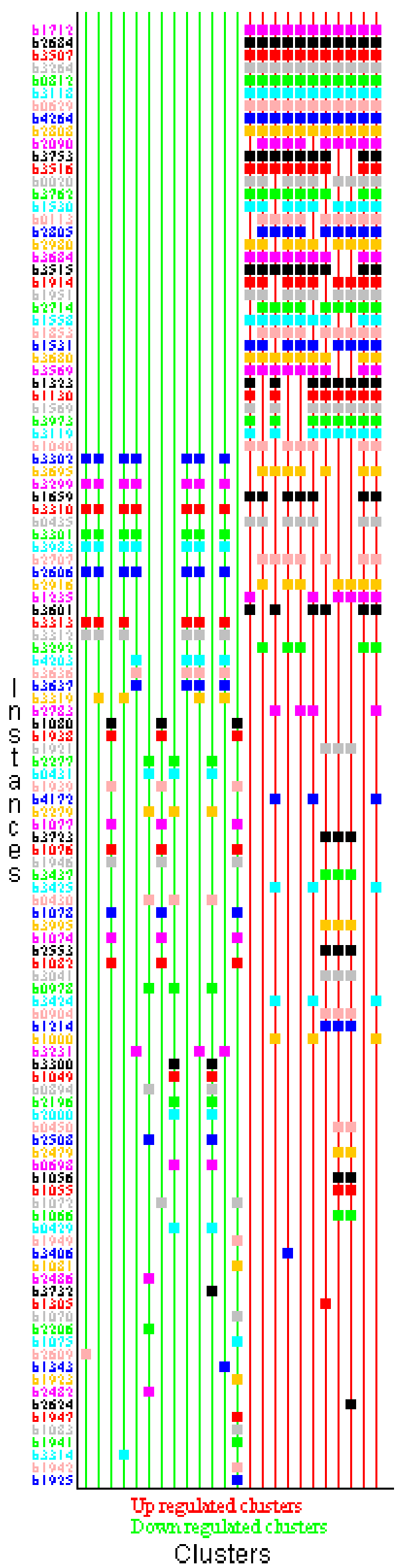

Figure 8.1: A visualisation for the clustering results of one data set 
Chapter 8: Tools

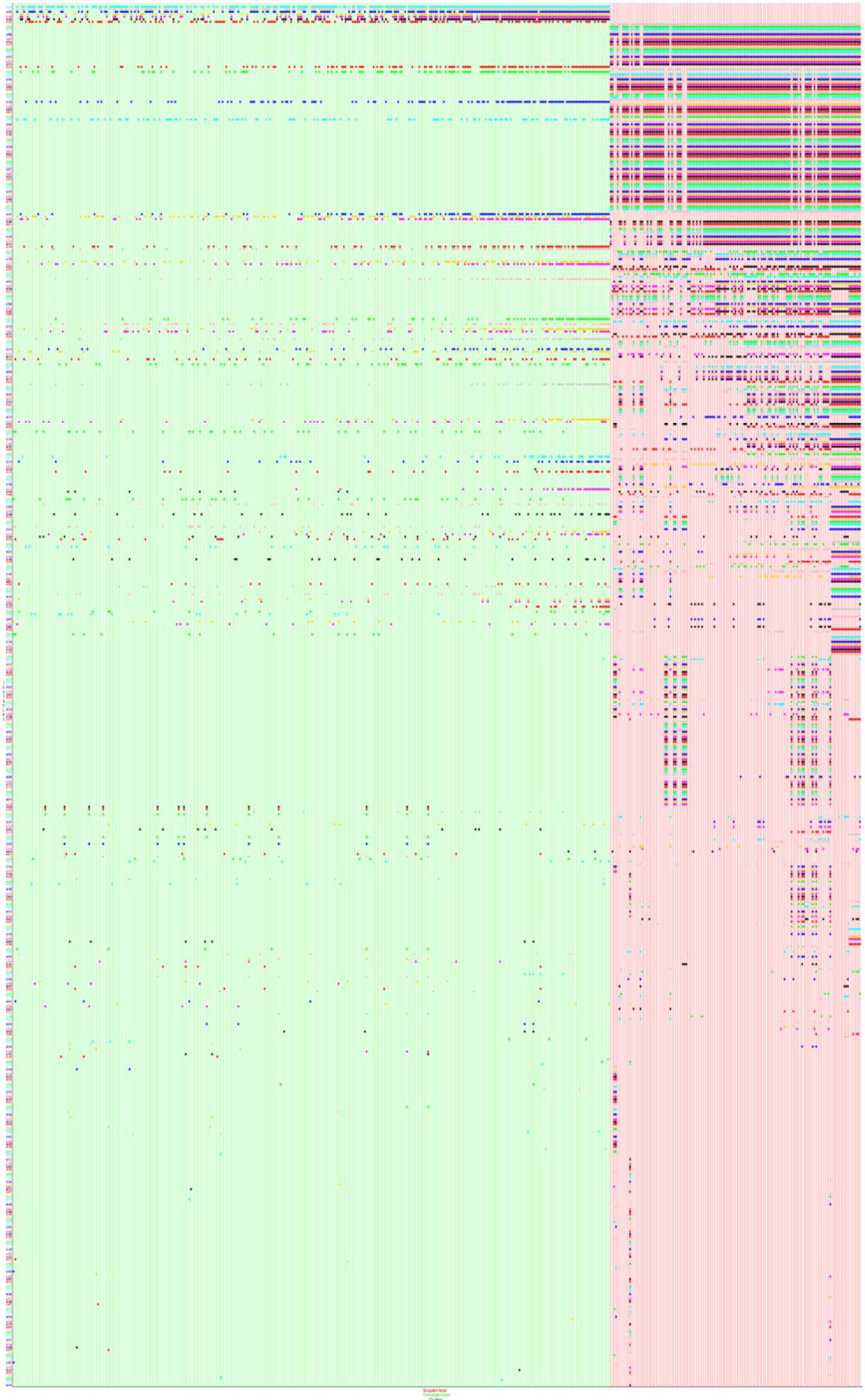

Figure 8.2: A visualisation for the clustering results of another data set 


\section{Chapter 9 Conclusion}

The clustering experiments we carried out through chapters three to seven showed that in general MAXCCLUS is doing a reasonably good job in clustering instances and characterising them to make it easy for the user (the scientist) to make sense of the microarray experimental data. The experiments also identified some limitations of MAXCCLUS, and showed that some improvements that we had expected would help did not in fact improve MAXCCLUS.

Although MAXCCLUS was able to describe, on average, only about $37 \%$ of the instances of the microarray experimental data (with a range between about $5 \%$ to about $79 \%$ ) using its simple generalisation approach (see section 2.3), we could not increase the number of instances described by MAXCCLUS by using an exhaustive search for generalisation. This could be because of the relatively high accuracy value (95\%) that was used in the clustering experiment (see Chapter 3). However, the exhaustive search generalisation was much more expensive than the simple generalisation.

MAXCCLUS depends on the significance test to select from the set of allclusters only the clusters that are statistically significant as specified by the user accuracy value (95\%). The clustering experiment of chapter four examined the significance test by using MAXCCLUS to cluster operons instead of genes. An operon is a group of genes that are functionally dependent on each other during a microarray experiment; therefore they have dependency relation. The result of the clustering experiment of chapter four showed that, when clustering genes, MAXCCLUS ignored the fact that some genes are dependent on other genes. Ignoring this fact led MAXCCLUS to 
select clusters that are big enough to be considered statistically significant but were not really significant. The MAXCCLUS significance test needs to be modified to consider the dependency of some genes on others, so that a cluster will be considered significant only if it consists of enough independent instances.

On the side of textual data, the clustering experiment of chapter five showed that it is advantageous to delete some of the descriptors of the instances providing that these descriptors are not domain-relevant ones. Deleting these descriptors let MAXCCLUS to create rules that are more scientifically informative, and to describe almost the same number of instances faster (only $74 \%$ as much time) compared with clustering with all the descriptors. On the other hand, the clustering experiment showed that when deleting more descriptors, including some domain-relevant ones, MAXCCLUS performed poorly, describing many fewer instances and finding fewer clusters than before deleting descriptors.

Also, on the side of textual data, the clustering experiment of chapter six showed that adding more descriptors (synonyms and/or hypernyms of already existing descriptors) to the descriptor sets of the instances did not improve MAXCCLUS clustering. After adding descriptors, MAXCCLUS behaved badly: it was not able to describe more instances than before adding descriptors, it created many alternative rules for clusters characterisations that can confuse the user who is trying to make sense of the clusters, and it took much longer to cluster. This result may be because the added synonyms and/or hypernyms retrieved from WordNet were for the non-domain descriptors only (because WordNet does not contain the domain-relevant words for genetics). It may be that MAXCCLUS would behave better if we could find the frequent synonyms and/or hypernyms for the domain-relevant descriptors. It might also be better to replace descriptors by the most frequent synonyms or hypernyms, rather than simply adding all synonyms or hypernyms, but this is a topic for future work. 
The clustering experiment of chapter seven showed that the instances that MAXCCLUS clusters need to have a clearly district categories with a gap between the categories. MAXCCLUS was not able to produce as good results when clustering using a continuous range of categories as when clustering using two categories separated with a clear gap. This behaviour is known for other clustering algorithms too. If one needs to use MAXCCLUS to cluster instances, one must be sure that these instances have clear gaps in between their categories.

The experiment also showed that there is a problem of scaling to larger number of instances with respect to clustering time. As MAXCCLUS attempts to cluster thousands of instances the time increases dramatically.

Note that it is the significance test, rather than the construction of the clusters that dominates the time. 


\section{Appendix}

\section{The words for the Bible in "Simple English"}

This appendix lists the words that were used to delete the descriptors using the first method in chapter 5 (deleting descriptors using the Bible words dictionary). There are 3561 words (about 1000 root words).

The list of words from WordNet (the second method used in chapter 5 for deleting descriptors) is too large to include (44,309 words), and would not be informative. 
Appendix: The words for the Bible in "Simple English"

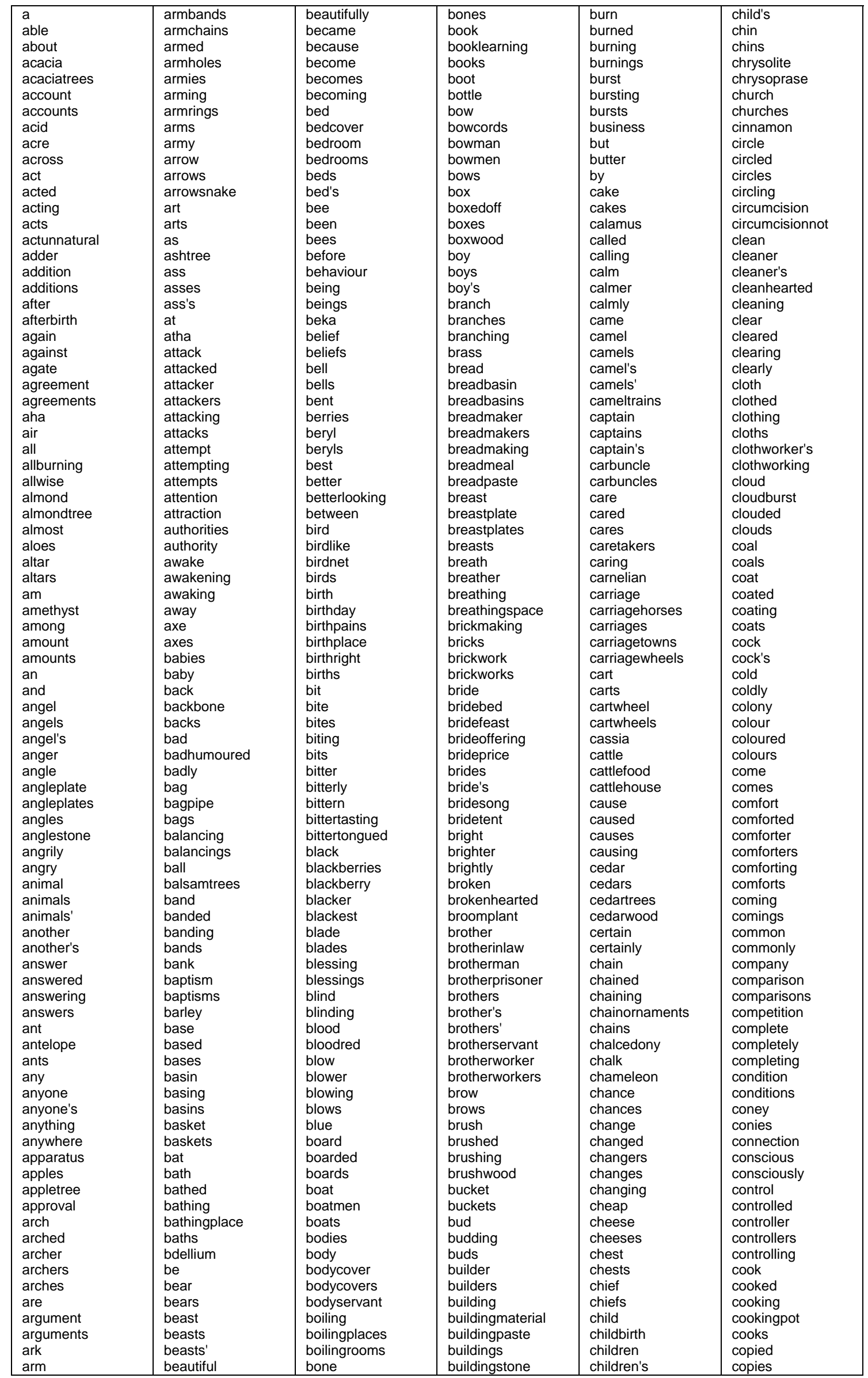


Appendix: The words for the Bible in "Simple English"

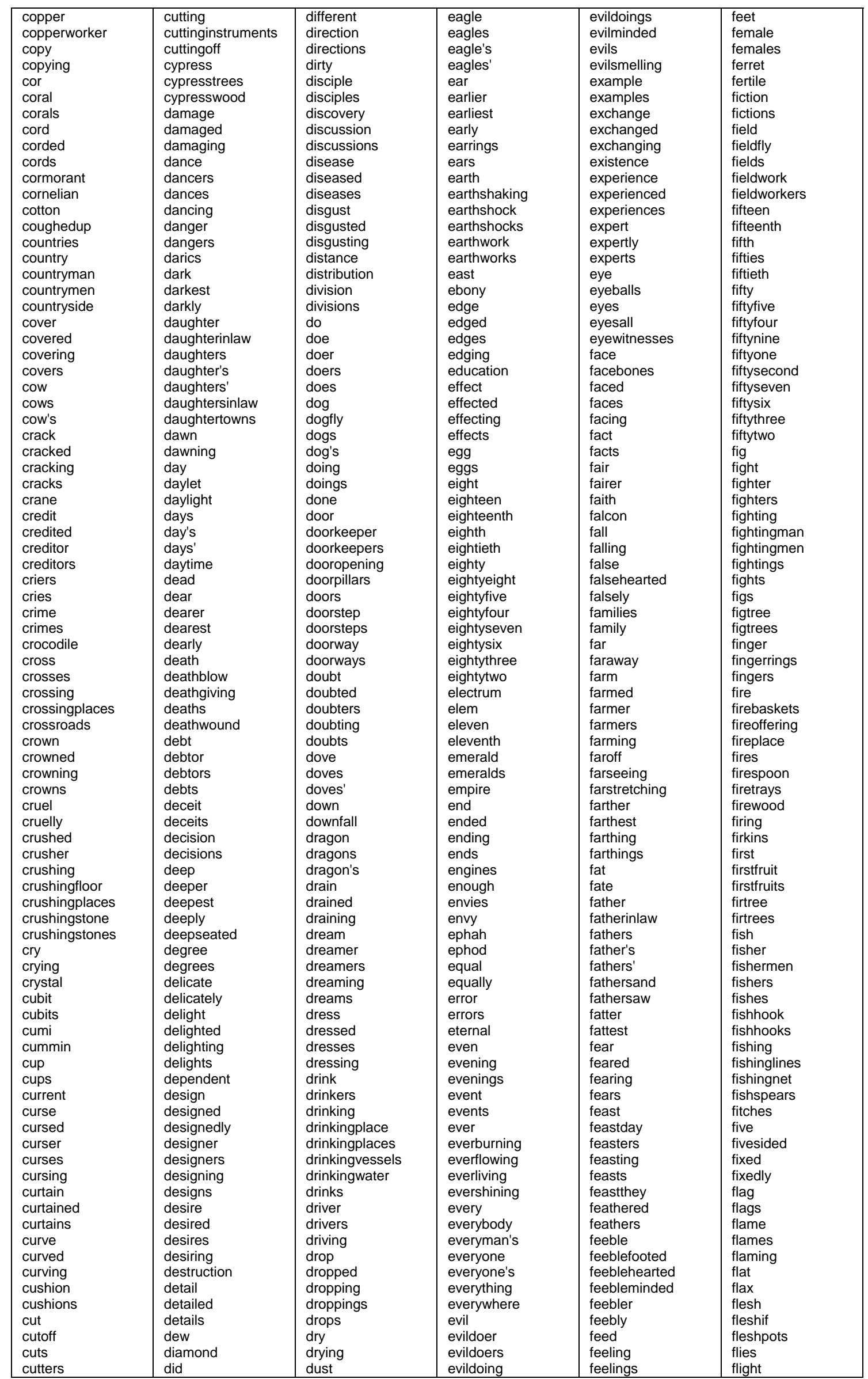


Appendix: The words for the Bible in "Simple English"

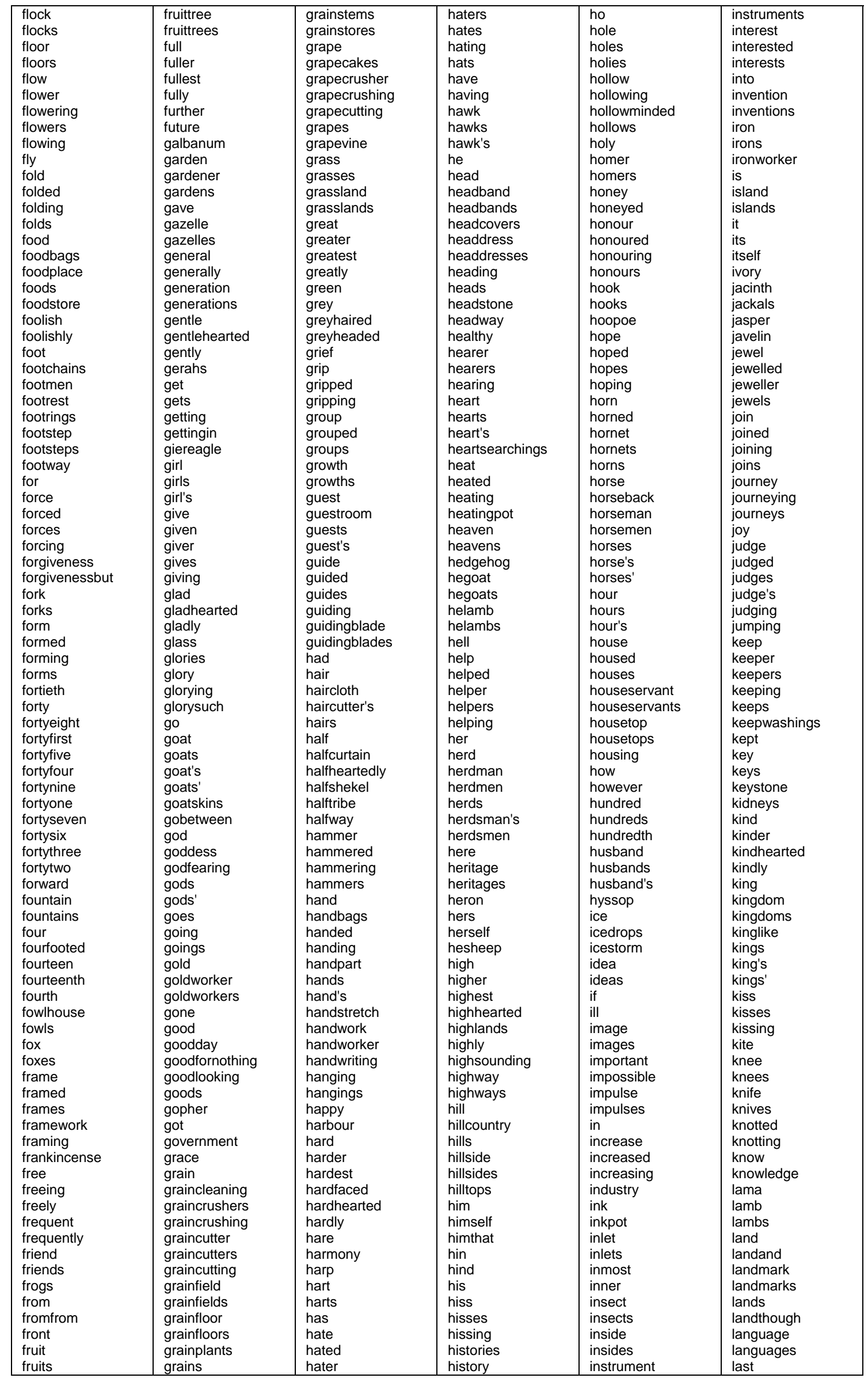


Appendix: The words for the Bible in "Simple English"

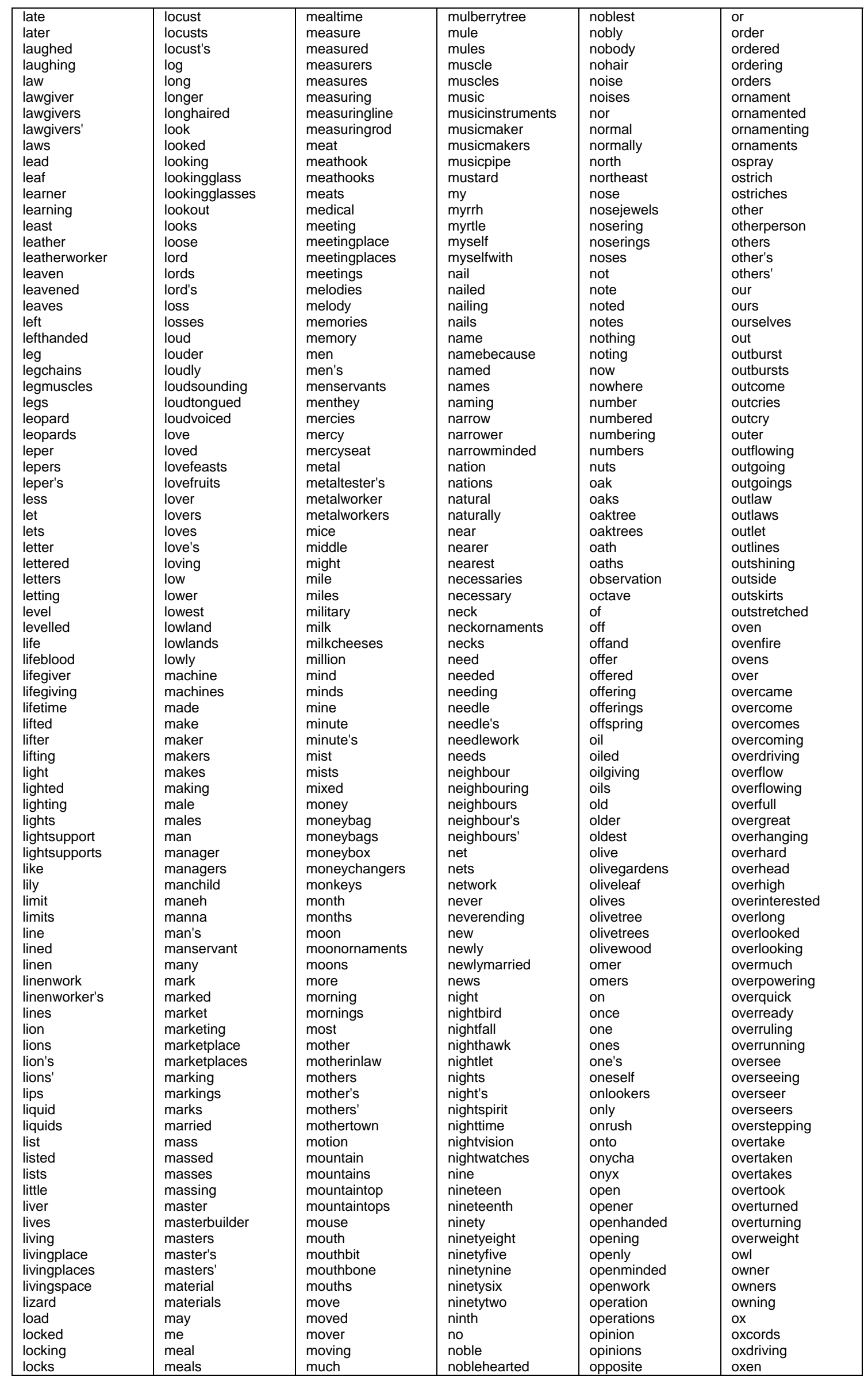


Appendix: The words for the Bible in "Simple English"

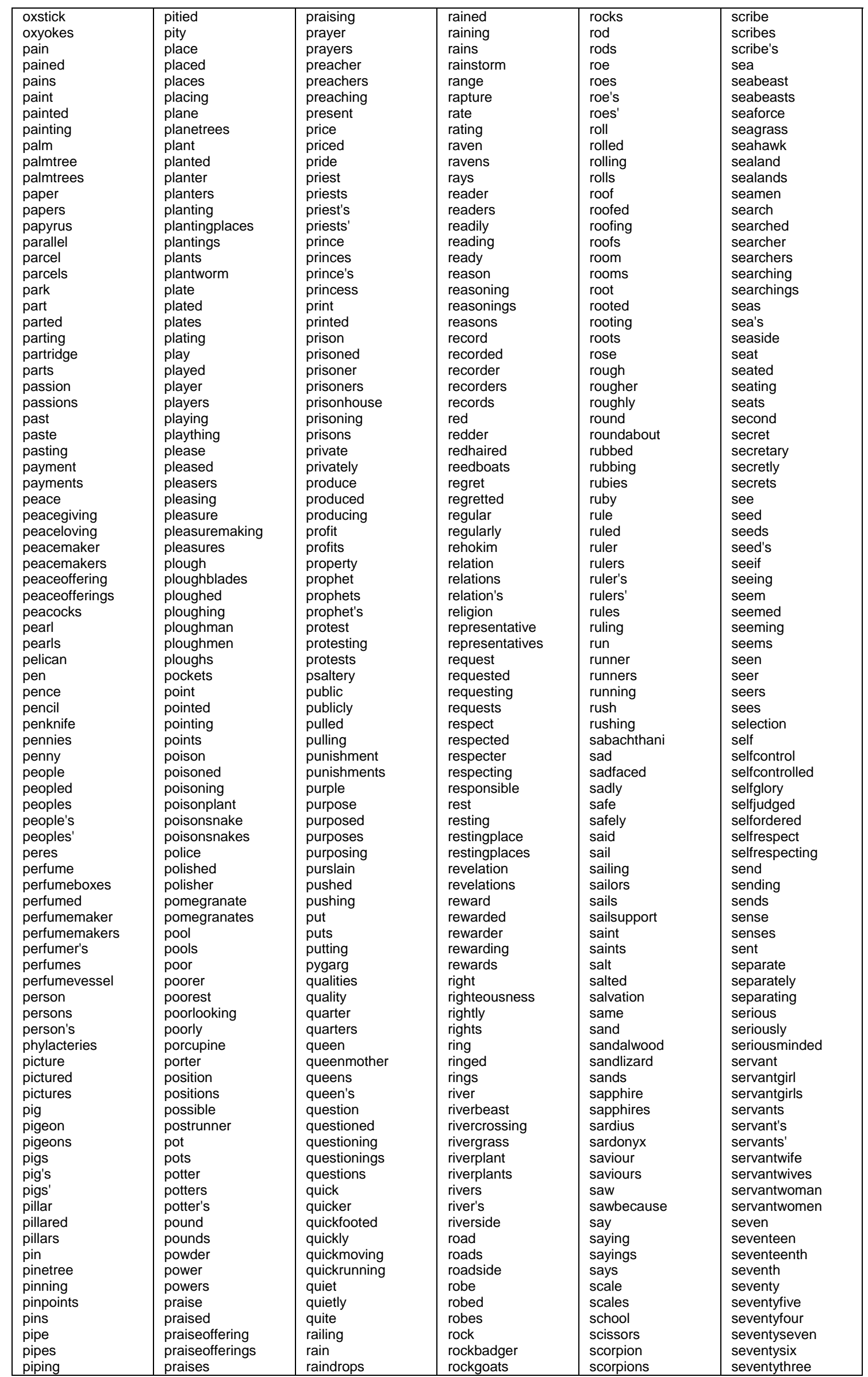


Appendix: The words for the Bible in "Simple English"

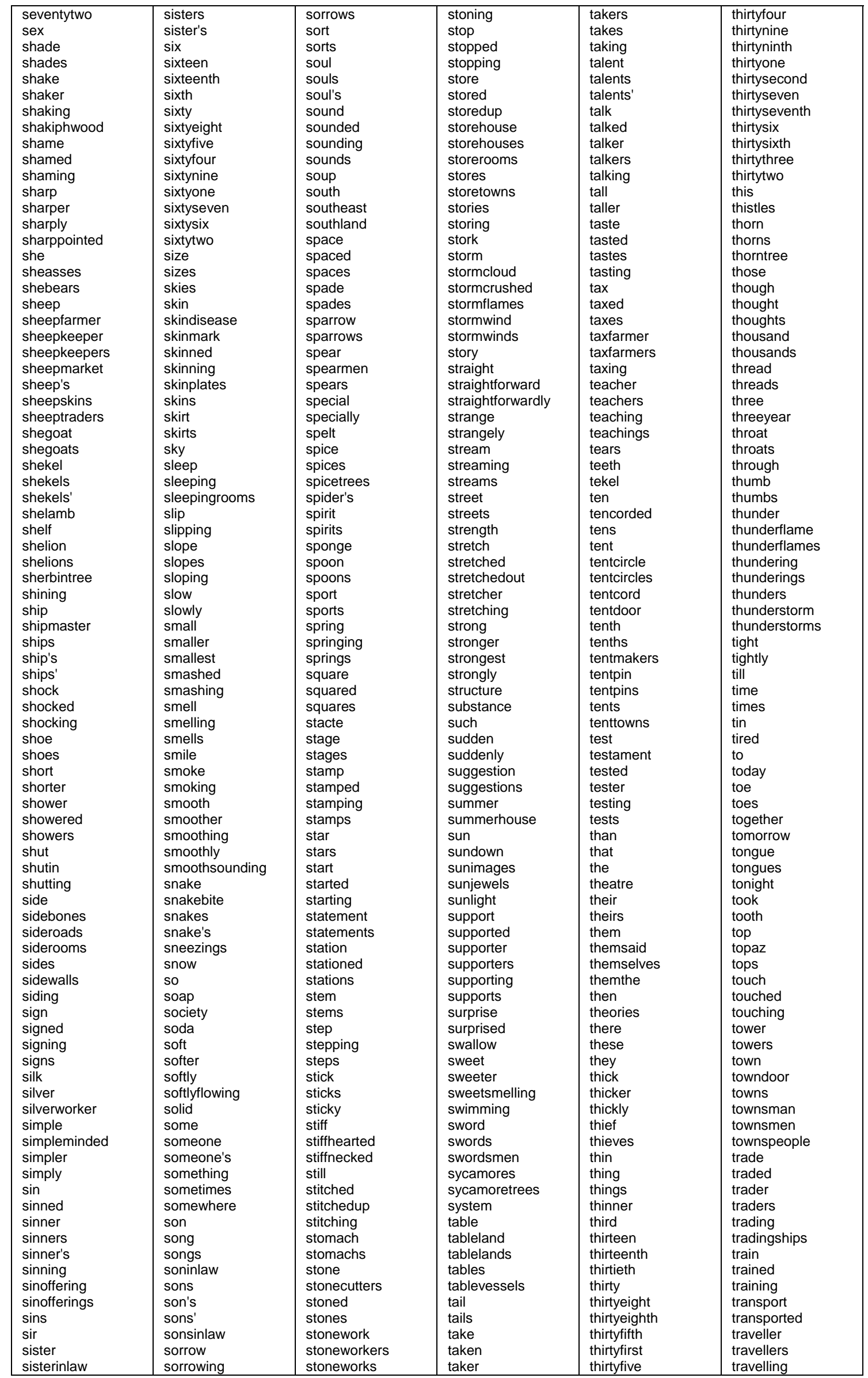


Appendix: The words for the Bible in "Simple English"

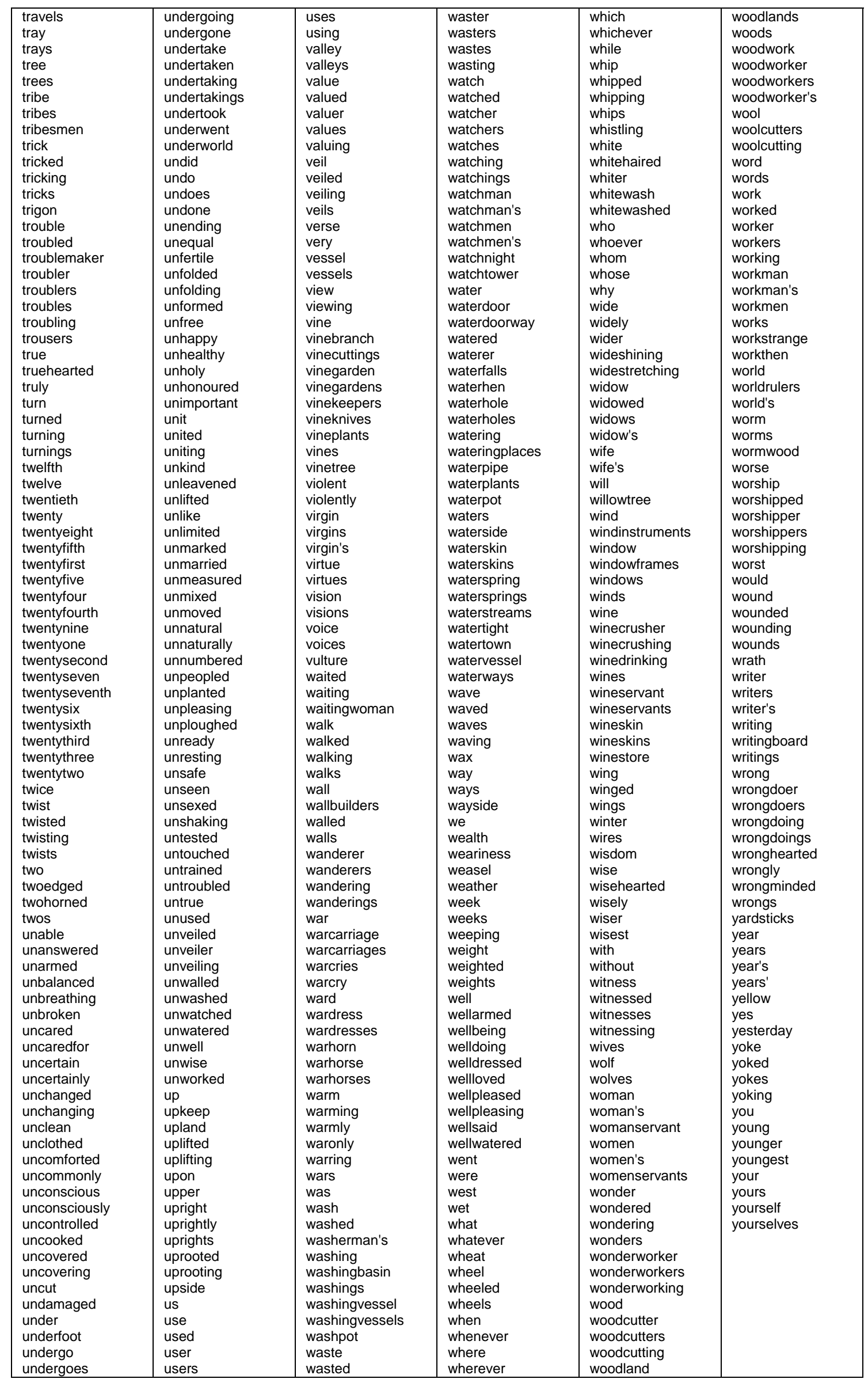


Appendix: The words for the Bible in "Simple English" 


\section{Bibliography}

Andreae, P; Shavlik, J; and Molla M. 2001

Characterising Genes From Gene-array Data.

A presentation talk.

http://www.mcs.vuw.ac.nz/ pondy/projects/maxcclus-talk.html

Bassett, D; Eisen, M; and Boguski, M. 1999

Gene expression informatics - it's all in your mine

nature genetics supplement, volume 21, january 1999

Bible in Basic English

http://www.biblestudytools.net/Information/BibleinBasicEnglish.html

Blaschke, C; Oliveros, J; and Valencia, A. 2001

Mining functional information associated with expression arrays

Funct Integr Genomics (2001) 1:256-268

Boeckmann, B; Bairoch, A; Apweiler, R; Blatter, M.C; Estreicher, A;

Gasteiger, E; Martin, M.J; Michoud, K; O'Donovan, C; Phan, I; Pilbout, S; and Schneider, M. 2003

The Swiss-Prot protein knowledgebase and its supplement TrEMBL in 2003.

Nucleic Acids Res. 31:365-370(2003).

Carr, D; Somogyi, R; and Michaels, G. 1999

Templates for Looking at Gene Expression Clustering

Statistical Computing \& Statistical Graphics Newsletter April 97, 20-29

Chen, Y; Bittner, M; and Dougherty, E. 1999

Issues associated with microarray data analysis and integration

Nature Genet. 22, 213-215; 1999

Claverie J-M. 1999

Computational Methods for the identification of

differential and coordinated gene expression

Human Molecular Genetics, 1999, Vol 8, No. 10, 1821-1832

D'haeseleer, P; Liang, S; and Somogyi, R. 1999

Gene Expression Data Analysis and Modeling

Tutorial

Session on Gene Expression and Genetic Networks

Pacific Symposium on Biocomputing, 1999

Hawaii , January 4-9, 1999

D'haeseleer, P; Liang, S; and Somogyi, R. 2000

Genetic network inference:

from co-expression clustering to reverse engineering

BIOINFORMATICS, Pages 707-726, Vol. 16, no. 8, 2000 
Falkenauer, E. and Marchand, A. 2001

Using k-Means? Consider ArrayMiner.

In Proceedings of the 2001 International Conference on Mathematics and

Engineering Techniques in Medicine and Biological Sciences

(METMBS'2001), Las Vegas, Nevada, USA, June 25-28, 2001.

Fasulo, D. 1999

An Analysis of Recent Work on Clustering Algorithms.

Technical Report \# 01-03-02

Department of Computer Science \& Engineering, University of Washington, Seattle, USA.

Komili, S. 2002

Clustering

Genomics \& Computational Biology, Section 6: October 29, 2002

Molla, M; Andreae, P; Glasner, J; Blattner, F. and Shavlik, J. 2002.

Interpreting Microarray Expression Data Using Text Annotating the Genes.

Information Scienses, 146, pp. 75-88.

Molla, M; Waddell, M; Page, D. and Shavlik, J. 2004.

Using Machine Learning to Design and Interpret Gene-Expression

Microarrays.

Al Magazine, 25, pp. 23-44.

Pellegrini, M. 2001

Computational methods for protein function analysis

Curr Opin Chem Biol. 2001 Feb;5(1):46-50.

Porter, M. F. 1980.

An algorithm for suffix stripping.

Program, Vol. 14, no. 3, pp 130-137, July 1980.

Princeton University, 2003

WordNet 2.0

Copyright $@ 2003$. All rights reserved.

http://www.cogsci.princeton.edu/ wn/

Rennie,J. 2002

WordNet::QueryData : a Perl module for accessing the WordNet database.

Copyright (c) 2000, 2001, 2002. All rights reserved.

http://www.ai.mit.edu/people/jrennie/WordNet

Sahami, M; Dumais, S; Heckerman, D; and Horvitz, E. 1998.

A Bayesian Approach to Filtering Junk E-mail.

AAAl'98 Workshop on Learning for Text Categorization, July 27, 1998,

Madison, Wisconsin. 
Smet, F; Mathys, J; Marchal, K; Thijs, G; Moor, B; and Moreau, Y. 2002 Adaptive quality-based clustering of gene expression profiles.

BIOINFORMATICS Vol. 18 no. 52002 Pages 735-746

Swiss-Prot

http://au.expasy.org/sprot/

Swiss-Prot User Manual

http://au.expasy.org/sprot/userman.html

Szabo, A; Boucher, K; Carroll, W; Klebanov, L; Tsodikov, A; and Yakovlev, A. Variable Selection and Pattern Recognition with Gene Expression Data Generated by the Microarray Technology

MATHEMATICAL BIOSCIENCES, 2002, 176, pp. 71-98

Tsodikov, A; Szabo, A; and Jones, D. 2002

Adjustments and measures of differential expression for microarray data.

BIOINFORMATICS Vol. 18 no. 22002 Pages 251-260

Turcato, D; Popowich, F; Toole, J; Fass, D; Nicholson, D; and Tisher, G.2000 Adapting a synonym database to specific domains.

In Proceedings of the ACL'2000.

Vilo, J; Brazma, A; Jonassen, I; Robinson, A; and Ukkonen, E. 2000

Mining for putative regulatory elements in the yeast genome

using gene expression data

ISMB-2000, August 2000, pages 384-394

Waddell, P. J. and Kishino, H. 2000

Cluster Inference Methods and Graphical Models

Evaluated on NCI60 Microarray Gene Expression Data

Genome Informatics 11: 129-140 (2000)

Williamson, D. 2002

Box and Whisker Diagrams: getting Microsoft Excel to plot them for you.

http://www.duncanwil.co.uk/boxplot.html

Copyright (c) 17 October 2002

Zhao, Y. and Karypis, G.

Clustering in Life Sciences

Technical Report

Department of Computer Science, University of Minnesota,

Minneapolis, MN 55455 